\title{
COMPENSATED CONVEXITY AND HAUSDORFF STABLE GEOMETRIC SINGULARITY EXTRACTIONS
}

\author{
KEWEI ZHANG \\ School of Mathematical Sciences, University of Nottingham, University Park, \\ Nottingham, NG7 2RD, UK \\ kewei.zhang@nottingham.ac.uk \\ ANTONIO ORLANDO \\ Inst. de Estructuras \& $\&$ Dept. de Mecánica, Universidad Nacional de Tucumán \\ Av. Independencia 1800, San Miguel de Tucumán, Argentina \\ aorlando@herrera.unt.edu.ar \\ ELAINE CROOKS \\ Department of Mathematics, Swansea University \\ Singleton Park, Swansea, SA2 8PP, UK \\ e.c.m.crooks@swansea.ac.uk \\ Received (Day Month Year) \\ Revised (Day Month Year) \\ Communicated by (xxxxxxxxxx)
}

We develop and apply the theory of lower and upper compensated convex transforms introduced in $[\mathrm{K}$. Zhang, Compensated convexity and its applications, Anal. Non-Lin. H. Poincaré Inst. 25 (2008) 743- 771] to define multiscale, parameterized, geometric singularity extraction transforms of ridges, valleys and edges of function graphs and sets in $\mathbb{R}^{n}$. These transforms can be interpreted as 'tight' opening and closing operators, respectively, with quadratic structuring functions. We show that these geometric morphological operators are invariant with respect to translation, and stable under curvature perturbations, and establish precise locality and tight approximation properties for compensated convex transforms applied to bounded functions and continuous functions. We moreover establish multiscale and Hausdorff stable versions of such transforms. Specifically, the stable ridge transforms can be used to extract exterior corners of domains defined by their characteristic functions. Examples of explicitly calculated prototype mathematical models are given, as well as some numerical experiments illustrating the application of these transforms to $2 d$ and $3 d$ objects.

Keywords: Compensated convex transforms; mathematical morphology; non-flat morphological operators; Moreau envelopes; ridges; valleys; edges; top-hat transform; locality property; Hausdorff stability.

AMS Subject Classification: 52A41, 49J52, 62H35, 14J17 


\section{Introduction}

In this paper we further develop the theory of compensated convex transforms introduced in Ref. 48 and apply this theory to geometric singularity extraction problems for singularities such as ridges, valleys, edges for general functions, images and geometric objects given by their characteristic functions in the Euclidean space $\mathbb{R}^{n}$. These geometric singularity extraction problems arise, for example, from signal, image and data processing, and computational geometry and computer-aided geometric design. ${ }^{18,41}$ We are particularly interested in geometric objects defined by their characteristic functions or by 'point clouds' and consequently, in the development of singularity extraction methods that are Hausdorff stable, that is, stable against dense random samples of the geometric object concerned.

In the present work, we will develop compensated convexity based, Hausdorff stable, multiscale global methods for extracting ridges, valleys, edges and exterior corners for functions and domains. Parallel to these theoretical developments, we have designed corresponding accurate and efficient numerical methods and schemes that are able to extract geometric singularities in two and three dimensional functions, images, shapes and data arrays. Here we focus on mathematical properties and refer to a follow-up paper ${ }^{50}$ for numerical algorithms.

Let us first recall the notions of quadratic compensated convex transforms defined in Ref. 48. We will consider functions $f: \mathbb{R}^{n} \mapsto \mathbb{R}$ meeting one of the following conditions for $x \in \mathbb{R}^{n}$
(L) $: f(x) \geq-A\left(1+|x|^{2}\right)$,
$(\mathbf{U}): f(x) \leq A\left(1+|x|^{2}\right)$
(B) : $|f(x)| \leq A\left(1+|x|^{2}\right)$,

for some constant $A \geq 0$. Later we simply refer to (L), (U) and (B) for these restrictions. Due to the locality property of compensated convex transforms ${ }^{48}$ and our practical concerns for applications to image and data processing, we in fact only need the function $f$ to be bounded, as data arrays have finite number of entries and we can always extend a function defined in a rectangular domain to the whole space by a constant value zero outside the domain.

Suppose $f: \mathbb{R}^{n} \mapsto \mathbb{R}$ satisfies (L). Then the quadratic lower compensated convex transform $^{48}$ (lower transform for short) for a given $\lambda>A$ is defined by

$$
C_{\lambda}^{l}(f)(x)=\operatorname{co}\left[\lambda|\cdot|^{2}+f\right](x)-\lambda|x|^{2} \quad x \in \mathbb{R}^{n},
$$

where $|x|$ is the Euclidean norm of $x \in \mathbb{R}^{n}$ and co $[g]$ the convex envelope ${ }^{23,34}$ of a function $g: \mathbb{R}^{n} \mapsto \mathbb{R}$ bounded below. Suppose $f: \mathbb{R}^{n} \mapsto \mathbb{R}$ satisfies (U). Then the quadratic upper compensated convex transform ${ }^{48}$ (upper transform for short) for a given $\lambda>A$ is defined by

$$
C_{\lambda}^{u}(f)(x)=\lambda|x|^{2}-\operatorname{co}\left[\lambda|\cdot|^{2}-f\right](x) \quad x \in \mathbb{R}^{n} .
$$

If $f: \mathbb{R}^{n} \mapsto \mathbb{R}$ satisfies (B) in (1.1), then the two quadratic mixed compensated convex transforms ${ }^{48}$ (mixed transforms for short) for given $\lambda>A$ and $\tau>A$ are defined respectively by $C_{\tau}^{u}\left(C_{\lambda}^{l}(f)\right)$ and $C_{\tau}^{l}\left(C_{\lambda}^{u}(f)\right)$. 
From definition (1.2), it also follows that ${ }^{\mathrm{a}} C_{\lambda}^{l}(f)(x)$ is the envelope of all the quadratic functions with fixed quadratic term $\lambda|x|^{2}$ that are less than or equal to $f$, that is,

$C_{\lambda}^{l}(f)(x)=\sup \left\{-\lambda|x|^{2}+\ell(x):-\lambda|y|^{2}+\ell(y) \leq f(y)\right.$ for all $y \in \mathbb{R}^{n}$ and $\ell$ affine $\}$,

whereas from (1.3) it follows that $C_{\lambda}^{u}(f)(x)$ is the envelope of all the quadratic functions with fixed quadratic term $\lambda|x|^{2}$ that are greater than or equal to $f$, that is,

$$
C_{\lambda}^{u}(f)(x)=\inf \left\{\lambda|x|^{2}+\ell(x): f(y) \leq \lambda|y|^{2}+\ell(y) \text { for all } y \in \mathbb{R}^{n} \text { and } \ell \text { affine }\right\}
$$

The compensated convex transforms as defined by (1.2) and (1.3) were introduced for the purpose of tight approximation of functions defined in $\mathbb{R}^{n}$ and their definition was motivated by the variational approach of material microstructure ${ }^{7,8}$ which led to an extensive study of the quasiconvex envelope (see Refs. 15, 48 and references therein). The 'tight' approximation property of lower and upper transforms is pivotal in our current work, because it provides a new mathematical tool for detecting singularities of functions that we exploit to detect features in images or data, remove noise from images, etc. It was established in Ref. 48, among other properties, that $C_{\lambda}^{l}(f)$ (respectively, $C_{\lambda}^{u}(f)$ ) is a 'tight' approximation from below (respectively, from above) in the sense that if $f$ is $C^{1,1}$ in a neighbourhood of $x_{0}$, there is then a finite $\Lambda>0$, such that $f\left(x_{0}\right)=C_{\lambda}^{l}(f)\left(x_{0}\right)$ (respectively, $f\left(x_{0}\right)=C_{\lambda}^{u}(f)\left(x_{0}\right)$ whenever $\lambda \geq \Lambda$. Furthermore, it was established in Ref. ${ }^{48}$ that given a compact set $K \subset \mathbb{R}^{n}$ the lower transform $C_{\lambda}^{l}\left(\operatorname{dist}^{2}(\cdot ; K)\right)$ of the squared-distance function $\operatorname{dist}^{2}(x ; K)$ and the upper transform $C_{\lambda}^{u}(f)(x)$ of a convex function satisfying (U) for $\lambda>A$ are both $C^{1,1}$ functions. Some explicit examples for compensated convex transforms have also been given in Ref. 49. The proofs of these regularity results used some methods from Ref. 9.

Lower and upper compensated convex transforms can be considered as parameterized semiconvex and semiconcave envelopes, respectively, for a given function. The notions of semiconvex and semiconcave functions go back at least to Reshetnyak $^{33}$ and have since been studied by many authors in different contexts (see, for example, Refs. 2, 3, 12, 29, 35, 46). Let $\Omega \subseteq \mathbb{R}^{n}$ be an open set, a function $f: \Omega \mapsto \mathbb{R} \cup\{+\infty\}$ is semiconvex if there is a constant $C \geq 0$ such that $f(x)=g(x)-C|x|^{2}$ with $g$ a convex function. More general weight functions, such as $|x| \sigma(|x|)$, for example, are also used in the literature for defining more general semiconvex functions. ${ }^{2,3,6,37,38}$ We do not discuss such generalised versions here.

Compensated convex transforms can also be viewed as critical mixed Moreau

${ }^{a}$ We are grateful to an anonymous referee of an earlier version of this manuscript to point out this characterization of the compensated convex transforms. 
envelopes $^{\mathrm{b}}$, given that

$$
C_{\lambda}^{l}(f)(x)=M^{\lambda}\left(M_{\lambda}(f)\right)(x), \quad C_{\lambda}^{u}(f)(x)=M_{\lambda}\left(M^{\lambda}(f)\right)(x),
$$

where the Moreau lower and upper envelopes ${ }^{31,32}$ are defined, in our notation, respectively, by

$$
\begin{aligned}
& M_{\lambda}(f)(x)=\inf \left\{f(y)+\lambda|y-x|^{2}, y \in \mathbb{R}^{n}\right\}, \\
& M^{\lambda}(f)(x)=\sup \left\{f(y)-\lambda|y-x|^{2}, y \in \mathbb{R}^{n}\right\},
\end{aligned}
$$

with $f$ meeting condition (L) and (U), respectively. Moreau envelopes play important roles in optimization, nonlinear analysis, optimal control and Hamilton-Jacobi equations, both theoretically and computationally. ${ }^{14,12,23,36}$ The mixed Moreau envelopes $M^{\tau}\left(M_{\lambda}(f)\right)$ and $M_{\tau}\left(M^{\lambda}(f)\right)$, also known as Lasry-Lions regularisations, ${ }^{29}$ have also been extensively studied and used as approximation and smoothing methods of not necessarily convex functions. ${ }^{5,12}$ In particular, in the partial differential equation literature, the focus of the study of the mixed Moreau envelopes has mainly been on the case $\tau>\lambda$, given that, for this case, and under suitable growth conditions, $M^{\tau}\left(M_{\lambda}(f)\right)$ and $M_{\tau}\left(M^{\lambda}(f)\right)$ are both $C^{1,1}$ functions ${ }^{5,12,29}$ but, crucially, not 'tight approximations' of $f$, in key contrast to our lower and upper transforms $C_{\lambda}^{l}(f)(x)$ and $C_{\lambda}^{u}(f)(x) .{ }^{48}$ Generalised inf and sup convolutions have also been considered, for instance in Refs. 12, 36.

Moreau lower and upper envelopes have also been employed in mathematical morphology in the 1990's, ${ }^{25,11}$ to define greyscale erosion and dilation morphological operators, whereas the critical mixed Moreau envelopes $M^{\lambda}\left(M_{\lambda}(f)\right)$ and $M_{\lambda}\left(M^{\lambda}(f)\right)$ are greyscale opening and closing morphological operators. ${ }^{44}$ If we denote by $b_{\lambda}(x)=-\lambda|x|^{2}$ the quadratic structuring function, introduced for the first time in Refs. 25, 10, then with the notation of Refs. 40, 44, we have

$$
\begin{aligned}
& M_{\lambda}(f)(x)=\inf _{y \in \mathbb{R}^{n}}\left\{f(y)-b_{\lambda}(y-x)\right\}=: f \ominus b_{\lambda}, \\
& M^{\lambda}(f)(x)=\sup _{y \in \mathbb{R}^{n}}\left\{f(y)+b_{\lambda}(y-x)\right\}=: f \oplus b_{\lambda}
\end{aligned}
$$

so that (1.6) can alternatively be written as

$$
C_{\lambda}^{l}(f)=\left(f \ominus b_{\lambda}\right) \oplus b_{\lambda} \quad \text { and } \quad C_{\lambda}^{u}(f)=\left(f \oplus b_{\lambda}\right) \ominus b_{\lambda} .
$$

The application of $M^{\lambda}\left(M_{\lambda}(f)\right)$ and $M_{\lambda}\left(M^{\lambda}(f)\right)$ in mathematical morphology, however, has not met with corresponding success, nor have its properties been fully explored. This is in contrast with the rôle, recognized since its introduction, that is played by paraboloid structuring functions in defining morphological scale-spaces in

\footnotetext{
${ }^{b}$ We are grateful to an anonymous referee of an earlier version of this manuscript to point out this identity.

'In convex analysis, the infimal convolution of $f$ with $g$ is denoted as $f \square g$ and is defined as $(f \square g)(x)=\inf _{y}\{f(y)+g(x-y)\} .{ }^{34}$ This is closely related to the erosion of $f$ by $g$, given that $(f \square g)(x)=f(x) \ominus(-g(-x))$.
} 
image analysis. ${ }^{25,10}$ For this and related topics concerning the morphological scalespace representation produced by quadratic structuring functions, we refer to the pionering works. ${ }^{25,10}$ Here, we would like only to observe that via the identity (1.6), we now have a direct characterization of the quadratic structuring based opening and closing morphological operators, either in terms of the convex envelope (see (1.2) and (1.3)) or in terms of envelope from below/above with parabolas (see (1.4) and (1.5)). Such characterizations will allow us to derive various new geometric and stability properties for the opening and closing morphological operators.

Given the interpretation (1.6) and the observation that lower and upper compensated convex transforms are also parameterized $\lambda$-semiconvex and $\lambda$-semiconcave envelopes of $f$, respectively, the compensated convex transforms become very useful geometric (curvature based) opening and closing morphological operators based on one-sided curvature restrictions. For instance, we have that, in the viscosity sense, the Hessians $D^{2} C_{\lambda}^{l}(f)(x) \geq-2 \lambda$ and $D^{2} C_{\lambda}^{u}(f)(x) \leq 2 \lambda$. As far as we know, the connections between these types of opening and closing morphological operators and the compensated convex transforms were not known before the present work.

When we apply compensated convex transforms to extract singularities from characteristic functions of compact geometric sets, our operations can be viewed as the application of morphological operations devised for 'greyscale images' to 'binary images'. A natural question would therefore be, what is the advantage of adopting such an approach, given that we are applying more involved operations for processing binary images, when in the current literature ${ }^{40,44}$ there are 'binary' set theoretic morphological operations that have been specifically designed for the tasks under examination. Some reasons are the following:

(1) Since compensated convex transforms of characteristic functions are (Lipschitz) continuous, applying a combination of transforms will produce a landscape of various levels (heights) that can be designed to highlight a specific type of singularity. We can then extract multiscale singularities by taking thresholds at different levels. In fact, the graphs of functions obtained by combinations of compensated convex transforms contain much more geometric information than binary operations that produce simply a yes or no answer. Also, for 'thin' geometric structures, such as curves and surfaces, it is difficult to design 'binary' morphological operations to be Hausdorff stable.

(2) We will show that the upper transform $E \mapsto C_{\lambda}^{u}\left(\chi_{E}\right)$ is Hausdorff-Lipschitz continuous, in the sense that for every $x \in \mathbb{R}^{n}$

$$
\left|C_{\lambda}^{u}\left(\chi_{E}\right)(x)-C_{\lambda}^{u}\left(\chi_{F}\right)(x)\right| \leq 2 \sqrt{\lambda} \operatorname{dist}_{\mathcal{H}}(E, F)
$$

for non-empty compact sets $E, F \subset \mathbb{R}^{n}$, where $\operatorname{dist}_{\mathcal{H}}(E, F)$ is the Hausdorff distance between two non-empty compact sets $E$ and $F$. Thus we can define transforms that are Hausdorff stable against sampling of geometric shapes. This helps us to deal with surfaces given by sampled 'point clouds' in $\mathbb{R}^{3}$.

(3) For many prototype geometric models, we can calculate our designed singularity 
extraction operations explicitly. We can then exploit the Hausdorff stability result mentioned above to prove that our methods also apply in more general cases.

(4) Although compensated convex transforms are defined for functions in $\mathbb{R}^{n}$, they can be easily adapted to image pixel settings. In this paper, we will not explicitly define our transforms on pixels, and rely on numerical schemes just for the convex envelope.

We proceed now to introduce the singularity extraction operations discussed in this paper using the notation and theory of compensated convex transforms.

The ridge, valley and edge transforms of scale $\lambda$ for $f: \mathbb{R}^{n} \mapsto \mathbb{R}$ are defined, respectively, by the following operations:

$$
\begin{aligned}
& R_{\lambda}(f)(x)=f(x)-C_{\lambda}^{l}(f)(x), \quad V_{\lambda}(f)(x)=f(x)-C_{\lambda}^{u}(f)(x), \\
& E_{\lambda}(f)(x)=C_{\lambda}^{u}(f)(x)-C_{\lambda}^{l}(f)(x),
\end{aligned}
$$

for $x \in \mathbb{R}^{n}$ and $\lambda>A$, with $f$ and $\lambda$ meeting the corresponding conditions listed in (1.1). Note that these are basic morphological operations ${ }^{44}: R_{\lambda}(f)$ is the 'top-hat' transform, i.e. $R_{\lambda}(f)=f-\left(\left(f \ominus b_{\lambda}\right) \oplus b_{\lambda}\right) ;-V_{\lambda}(f)$ is the 'bottom-hat' transform, i.e. $-V_{\lambda}(f)=\left(\left(f \oplus b_{\lambda}\right) \ominus b_{\lambda}\right)-f$, and $E_{\lambda}(f)$ is the set-complementary top-hat morphological operator, being the arithmetic difference between the closing and the opening of $f$, i.e. $E_{\lambda}(f)=\left(\left(f \oplus b_{\lambda}\right) \ominus b_{\lambda}\right)-\left(\left(f \ominus b_{\lambda}\right) \oplus b_{\lambda}\right)$. Their definition in terms of compensated convex transforms, and the exploitation of some basic properties of these transforms, permits a relatively easy evaluation of some of their geometric properties and of how they respond to the different types of singularities. Specifically, we will show that the transforms are invariant with respect to translation, stable under curvature perturbation and enjoy precise locality and tight approximation properties by giving explicit estimates.

We will then establish the key property that the upper compensated convex transform of characteristic functions is Hausdorff-Lipschitz continuous as mentioned above. This central result underpins our definition of the stable ridge transform for characteristic functions $\chi_{E}$ of a non-empty compact set $E \subset \mathbb{R}^{n}$, namely

$$
S R_{\tau, \lambda}\left(\chi_{E}\right):=C_{\lambda}^{u}\left(\chi_{E}\right)-C_{\tau}^{l}\left(C_{\lambda}^{u}\left(\chi_{E}\right)\right)
$$

which is the top-hat transform of the closing operator $C_{\lambda}^{u}\left(\chi_{E}\right)$. Taking into account the identities (1.9), we therefore have

$$
S R_{\tau, \lambda}\left(\chi_{E}\right)=\left(f \oplus b_{\lambda}\right) \ominus b_{\lambda}-\left(\left(\left(f \oplus b_{\lambda}\right) \ominus b_{\lambda}\right) \ominus b_{\tau}\right) \oplus b_{\tau} .
$$

The Hausdorff-Lipschitz continuity property of the upper transform enables us to prove that this transform is Hausdorff-Lipschitz stable, and we then verify that this operator is able to extract the boundary and exterior corners of a domain. We show that the stable ridge transform at a regular point $x_{0} \in \partial K$ has the fixed value $S R_{\lambda, \tau}\left(\chi_{K}\right)\left(x_{0}\right)=(\sqrt{\lambda+\tau}-\sqrt{\tau})^{2} / \lambda$, whereas for a prototype example of an exterior corner $x_{0}$, the value of $S R_{\lambda, \tau}\left(\chi_{K}\right)\left(x_{0}\right)$ is greater than the 
one attained at all regular points. By using the prototype example as a tool, we therefore establish sufficient conditions for the extraction of exterior corners for a domain. This provides a filter for extracting exterior corners defined by $\left\{x \in \mathbb{R}^{n}: S R_{\lambda, \tau}\left(\chi_{K}\right)(x)>(\sqrt{\lambda+\tau}-\sqrt{\tau})^{2} / \lambda\right\}$.

In current image processing practice, numerous methods for extraction of ridges, valleys and edges have been proposed. ${ }^{20,30,39}$ Examples include techniques that rely on the search for local maximum and minimum along one direction such as the ones proposed in Ref. 22, 17; methods that use an approximation of the gradient or of the Hessian of the underlying function, such as the Sobe ${ }^{43}$ and the Canny edge detectors, ${ }^{13}$ or the Harris corner detector ${ }^{24}$; procedures that are integral based edge and corner filters such as the Yaroslavsky filter, ${ }^{47}$ the SUSAN filter ${ }^{42}$ and the bilateral filter ${ }^{45}$; or based on the succesive application of different morphological operators such as the morphological corner detector introduced in Ref. 28 which performs the dilation of an image using a given structuring element followed by an erosion using another structuring element. The effectiveness of these methods is mainly justified on the basis of numerical experiments, and to our knowledge, at present none of the existing techniques has been proved to be Hausdorff stable. On the other hand, Hausdorff stability is particularly important when one needs to extract features of 'point clouds' representing sampled domains. If a geometric shape is densely sampled, from the human vision point of view, one can still identify geometric features of the sample and sketch its boundary. However, from the mathematical and computing science perspective, the identification of such features poses a great challenge. So the development of Hausdorff stable approaches to ridge, valley and edge detection is highly desirable.

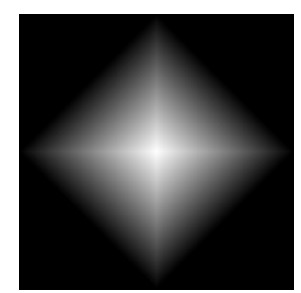

(a)

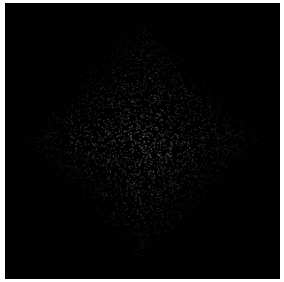

(b)

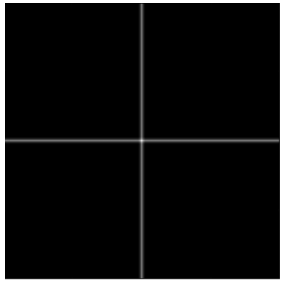

(c)

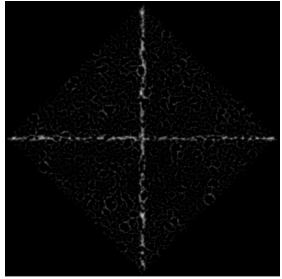

$(d)$

Fig. 1. (a) Image of $f(x, y)$; (b) Sampled image of $f(x, y)$ by random salt and pepper noise; $(c)$ Ridges of $f(x, y) ;(d)$ Ridges from sampled image.

To illustrate the application of our stable ridge transform, consider the scaled image of the 'tent' function $f(x, y)=\max \{0,1-\max \{|x|,|y|\}\}$, with $|x| \leq 1$ and $|y| \leq 1$, and its sparse sampling - see Figure 1(a), (b) respectively. Due to the Hausdorff stability of the stable ridge transform, we are able to recover an approximation of the ridges from the sampled image - compare Figure $1(c),(d)$.

The plan of the paper is as follows. In Section 2 we present our notation, recall 
some known results of convex analysis, and state basic properties of compensated convex transforms. In Section 3 we describe further properties of the compensated convex transforms, such as the Expansion Theorem and locality and density results. Such properties are fundamental for the design and understanding of the behaviour of the ridge, valley and edge transforms, which are introduced and analysed in Section 4 . Section 5 presents the stable ridge transform and contains the main result on its Hausdorff stability. The behaviour of this operator is then verified on some prototype examples in Section 6. We conclude the paper with Section 7 that details the proofs of the main results.

\section{Notation and Preliminaries}

We first collect together some results and definitions from convex analysis for functions $f$ taking finite values, i.e. for $f: \mathbb{R}^{n} \mapsto \mathbb{R}$, and refer to Refs. 23, 34 for further references and proofs. We then list some basic properties of compensated convex transforms, some of which can be established with the help of the characterization (1.6). This section is concluded with the definition of some types of regularity of a domain and its boundary points.

Proposition 2.1. Let $f: \mathbb{R}^{n} \mapsto \mathbb{R}$ be coercive in the sense that $f(x) /|x| \rightarrow \infty$ as $|x| \rightarrow \infty$, and $x_{0} \in \mathbb{R}^{n}$. Then

(i) The value co $[f]\left(x_{0}\right)$ of the convex envelope of $f$ at $x_{0} \in \mathbb{R}^{n}$ is given by

$$
\begin{gathered}
\operatorname{co}[f]\left(x_{0}\right)=\inf _{i=1, \ldots, n+1}\left\{\sum_{i=1}^{n+1} \lambda_{i} f\left(x_{i}\right): \sum_{i=1}^{n+1} \lambda_{i}=1, \sum_{i=1}^{n+1} \lambda_{i} x_{i}=x_{0},\right. \\
\left.\lambda_{i} \geq 0, x_{i} \in \mathbb{R}^{n}\right\} .
\end{gathered}
$$

If in addition, $f$ is lower semicontinuous, the infimum is reached by some $\left(\lambda_{i}^{*}, x_{i}^{*}\right)$ for $i=1,2, \ldots, n+1$ with $\left(x_{i}^{*}, f\left(x_{i}^{*}\right)\right)$ 's lying in the intersection of a supporting plane of the epigraph of $f$, epi $(f)$, and epi $(f)$.

(ii) The value co $[f]\left(x_{0}\right)$, for $f$ taking only finite values, can also be obtained as follows:

$$
\operatorname{co}[f]\left(x_{0}\right)=\sup \left\{\ell\left(x_{0}\right): \ell \text { affine and } \ell(y) \leq f(y) \text { for all } y \in \mathbb{R}^{n}\right\}
$$

with the sup attained by an affine function $\ell^{*} \in \operatorname{Aff}\left(\mathbb{R}^{n}\right)$.

We will also introduce the following local version of convex envelope at a point.

Definition 2.2. Let $r>0$ and $x_{0} \in \mathbb{R}^{n}$. Suppose $f: \bar{B}\left(x_{0} ; r\right) \mapsto \mathbb{R}$ is a bounded function in $\bar{B}\left(x_{0} ; r\right)$. Then the value $\operatorname{co}_{\bar{B}\left(x_{0} ; r\right)}[f]\left(x_{0}\right)$ of the local convex envelope 
of $f$ at $x_{0}$ in $\bar{B}\left(x_{0} ; r\right)$ is defined by

$$
\begin{aligned}
\mathrm{Co}_{\bar{B}\left(x_{0} ; r\right)}[f]\left(x_{0}\right)=\inf _{i=1, \ldots, n+1}\left\{\sum_{i=1}^{n+1} \lambda_{i} f\left(x_{i}\right):\right. & \sum_{i=1}^{n+1} \lambda_{i}=1, \sum_{i=1}^{n+1} \lambda_{i} x_{i}=x_{0}, \\
& \left.\lambda_{i} \geq 0,\left|x_{i}-x_{0}\right| \leq r, x_{i} \in \mathbb{R}^{n}\right\} .
\end{aligned}
$$

The difference between the local convex envelope defined here and the global convex envelope in Proposition 2.1 is that we only allow the infimum of convex combinations to be taken locally in $\bar{B}\left(x_{0} ; r\right)$. We need this notion for deriving the locality and density estimates for compensated convex transforms later in Section 3 .

The next result will enable us to extend some of our prototype examples to higher dimensional spaces through rotation. A proof of this result can be easily obtained by using the characterization of the convex envelope as biconjugate $f^{* *}$ (i.e. the convex conjugate of the convex conjugate) of the function $f$.

Proposition 2.3. (Partial Rotation Invariance property) Let $f(x, y)$ be a bounded function for $(x, y) \in \mathbb{R}^{2}$ such that $f$ is even in $y \in \mathbb{R}$, that is, for all $(x, y) \in \mathbb{R}^{2}$, $f(x, y)=f(x,-y)$. Let $F: \mathbb{R} \times \mathbb{R}^{n} \mapsto \mathbb{R}$ be defined by $F(x, z)=f(x,|z|)$ for $x \in \mathbb{R}$ and $z \in \mathbb{R}^{n}$. Then

$$
\mathrm{co}_{\mathbb{R}^{n+1}}[F](x, z)=\mathrm{co}_{\mathbb{R}^{2}}[f](x,|z|),
$$

where $\mathrm{co}_{\mathbb{R}^{n+1}}[F]$ and $\mathrm{co}_{\mathbb{R}^{2}}[f]$ are the convex envelopes of $F$ and $f$ in $\mathbb{R}^{n+1}$ and $\mathbb{R}^{2}$, respectively.

The following is an estimate of the Lipschitz constant for a convex function ${ }^{23,27}$ that will be used in the proof of Theorem 3.13.

Proposition 2.4. Suppose $g$ is a bounded convex function in $\bar{B}\left(x_{0} ; 2 r\right)$, then

$$
\operatorname{Lip}\left(g, B\left(x_{0} ; r\right)\right) \leq \frac{\operatorname{osc}\left(g, B\left(x_{0} ; 2 r\right)\right)}{r},
$$

where $\operatorname{Lip}\left(g, B\left(x_{0} ; r\right)\right)$ is the Lipschitz constant of $g$ in $B\left(x_{0} ; r\right)$ and $\operatorname{osc}\left(g, B\left(x_{0} ; r\right)\right)$ is the oscillation of $g$ in $B\left(x_{0} ; r\right)$ defined by $\operatorname{osc}\left(g, B\left(x_{0} ; r\right)\right)=\max _{B\left(x_{0} ; r\right)} g-\min _{B\left(x_{0} ; r\right)} g$.

Definition 2.5. Given a non-empty subset $E$ of $\mathbb{R}^{n}$ and $\delta>0$, we define the $\delta$-neighbourhood $E^{\delta}$ of $E$ by

$$
E^{\delta}=\left\{x \in \mathbb{R}^{n}: \operatorname{dist}(x ; E)<\delta\right\},
$$

where $\operatorname{dist}(x ; E)=\inf \{|x-y|, y \in E\}$.

Note that $E^{\delta}$ is an open subset of $\mathbb{R}^{n}$.

Definition 2.6. Let $E, F$ be non-empty subsets of $\mathbb{R}^{n}$. The Hausdorff distance between $E$ and $F$ is defined in Ref. 4 by

$$
\operatorname{dist}_{\mathcal{H}}(E, F)=\inf \left\{\delta>0: F \subset E^{\delta} \text { and } E \subset F^{\delta}\right\} .
$$


For a non-empty subset $E$ of $\mathbb{R}^{n}$, its characteristic function $\chi_{E}$ is lower (respectively, upper) semicontinuous if and only if $E$ is an open (respectively, closed) set. Furthermore, we can easily check that $\underline{\chi}_{E}=\chi_{\mathbb{R}^{n} \backslash\left(\overline{\mathbb{R}^{n} \backslash E}\right)}$, (see Ref. 26 at pag.211), where $f$ is the lower semicontinuous envelope of $f$.

We now list some properties of the quadratic compensated convex transforms and their relation to Moreau envelopes. This relation can be exploited to state certain convergence and regularity results for compensated convex transforms, e.g. the approximation results in Theorem 2.12 below. The following ordering properties can be found in Ref. 48 and are easy to prove:

$$
M_{\lambda}(f)(x) \leq C_{\lambda}^{l}(f)(x) \leq f(x) \leq C_{\lambda}^{u}(f)(x) \leq M^{\lambda}(f)(x) \quad\left(\text { for } x \in \mathbb{R}^{n}\right),
$$

and for $f \leq g$ in $\mathbb{R}^{n}$,

$$
C_{\lambda}^{l}(f)(x) \leq C_{\lambda}^{l}(g)(x) \quad \text { and } \quad C_{\lambda}^{u}(f)(x) \leq C_{\lambda}^{u}(g)(x) \quad\left(\text { for } x \in \mathbb{R}^{n}\right) .
$$

The following result is a special case of Theorem 3.5.8 at pag. 72 of Ref. 12 .

Proposition 2.7. Suppose $f: \mathbb{R}^{n} \mapsto \mathbb{R}$ is a Lipschitz function, then

$$
\begin{aligned}
& \lim _{\lambda \rightarrow \infty} M_{\lambda}(f)(x)=\lim _{\lambda \rightarrow \infty} M^{\lambda}(f)(x)=f(x), \\
& \lim _{\lambda, \tau \rightarrow \infty} M^{\tau}\left(M_{\lambda}(f)\right)(x)=\lim _{\lambda, \tau \rightarrow \infty} M_{\tau}\left(M^{\lambda}(f)\right)(x)=f(x)
\end{aligned}
$$

uniformly in $\mathbb{R}^{n}$.

For completeness, we also recall the following properties, which can be derived directly from corresponding ones of the convex envelope and using the definition of the compensated convex transforms.

Proposition 2.8. Let $f: \mathbb{R}^{n} \mapsto \mathbb{R}$ be nonnegative and meeting Condition (L) in (1.1). Then, for any $\lambda>A$, there holds

$$
C_{\lambda}^{l}(f)(x) \geq 0 \quad\left(\text { for } \quad x \in \mathbb{R}^{n}\right) .
$$

Proposition 2.9. Let $f, g$ be two real valued functions defined in $\mathbb{R}^{n}$ and meeting the conditions (1.1) with corresponding positive constants $A_{f}$ and $A_{g}$. Then for $\lambda, \mu \geq \max \left\{A_{f} A_{g}\right\}$ and $x \in \mathbb{R}^{n}$, there holds

$$
\begin{aligned}
& C_{\lambda+\mu}^{l}(f+g)(x) \geq C_{\lambda}^{l}(f)(x)+C_{\mu}^{l}(g)(x) \quad \text { and } \\
& C_{\lambda+\mu}^{u}(f+g)(x) \leq C_{\lambda}^{u}(f)(x)+C_{\mu}^{u}(g)(x) .
\end{aligned}
$$

Proposition 2.10. (Translation invariance property) For any $f: \mathbb{R}^{n} \mapsto \mathbb{R}$ bounded below and for any affine function $\ell: \mathbb{R}^{n} \mapsto \mathbb{R}, \operatorname{co}[f+\ell]=\mathrm{co}[f]+\ell$. Consequently, both $C_{\lambda}^{u}(f)$ and $C_{\lambda}^{l}(f)$ are translation invariant against the weight function, that is

$$
\begin{aligned}
& C_{\lambda}^{l}(f)(x)=\operatorname{co}\left[\lambda\left|(\cdot)-x_{0}\right|^{2}+f\right](x)-\lambda\left|x-x_{0}\right|^{2}, \\
& C_{\lambda}^{u}(f)(x)=\lambda\left|x-x_{0}\right|^{2}-\operatorname{co}\left[\lambda\left|(\cdot)-x_{0}\right|^{2}-f\right](x)
\end{aligned}
$$


for all $x \in \mathbb{R}^{n}$ and for every fixed $x_{0}$. Hence, at $x_{0}$,

$$
C_{\lambda}^{l}(f)\left(x_{0}\right)=\operatorname{co}\left[\lambda\left|(\cdot)-x_{0}\right|^{2}+f\right]\left(x_{0}\right), \quad C_{\lambda}^{u}(f)\left(x_{0}\right)=-\operatorname{co}\left[\lambda\left|(\cdot)-x_{0}\right|^{2}-f\right]\left(x_{0}\right) .
$$

Next we state some results on the rate of approximation of the lower and upper transforms under weaker regularity assumptions than those given in Theorem 2.3(iv) of Ref. 48, where $f$ was required to be locally of class $C^{1,1}$. We give first the following definitions.

Definition 2.11. Suppose $f: \mathbb{R}^{n} \mapsto \mathbb{R}$ and let $x_{0} \in \mathbb{R}^{n}$.

(i) We say that $f$ is locally $C^{\alpha}$ at $x_{0}$ for some $0<\alpha \leq 1$ if there are constants $\delta>0$ and $L>0$ such that when $0<\alpha \leq 1$,

$$
\left|f(x)-f\left(x_{0}\right)\right| \leq L\left|x-x_{0}\right|^{\alpha} \quad \text { whenever }\left|x-x_{0}\right| \leq \delta .
$$

(ii) We say that $f$ is locally $C^{\alpha}$ at $x_{0}$ for some $1<\alpha<2$ if $f$ is differentiable in the closed ball $\bar{B}\left(x_{0} ; \delta\right)$ and there are constants $\delta>0, L>0$ such that

$$
\left|D f(x)-D f\left(x_{0}\right)\right| \leq L\left|x-x_{0}\right|^{\alpha-1} \quad \text { whenever }\left|x-x_{0}\right| \leq \delta .
$$

(iii) For $\alpha=1$, we say that $f$ is locally Lipschitz at $x_{0}$ if

$$
\left|f(x)-f\left(x_{0}\right)\right| \leq L\left|x-x_{0}\right| \quad \text { whenever }\left|x-x_{0}\right| \leq \delta .
$$

We then have the following estimates that are stated here only for the lower transforms; similar results hold for upper transforms. Note that some of these estimates can be established by exploiting the connections between our lower and upper transforms and Moreau envelopes and developing ideas from Lemma 3.5.2, Theorem 3.5.3, Lemma 3.5.7 and Theorem 3.5.8 of Ref. 12. See Section 7 for more details.

\section{Theorem 2.12.}

(i) Suppose $f: \mathbb{R}^{n} \mapsto \mathbb{R}$ is lower semicontinuous, $f$ maps bounded sets to bounded sets and satisfies Condition ( $L$ ). If at some $x_{0} \in \mathbb{R}^{n}, f$ is locally $C^{\alpha}$ at $x_{0}$ for $0<\alpha<2$, then for $\lambda>0$ sufficiently large and $L$ of Definition 2.11,

$$
C_{\lambda}^{l}(f)\left(x_{0}\right) \leq f\left(x_{0}\right) \leq C_{\lambda}^{l}(f)\left(x_{0}\right)+L^{2 /(2-\alpha)}\left(\frac{\alpha}{2 \lambda}\right)^{\alpha /(2-\alpha)}\left(1-\frac{\alpha}{2}\right) .
$$

(ii) Suppose $f: \mathbb{R}^{n} \mapsto \mathbb{R}$ is lower semicontinuous, $f$ maps bounded sets to bounded sets and satisfies Condition $(\boldsymbol{L})$. If at some $x_{0} \in \mathbb{R}^{n}, f$ is differentiable, then for any $\epsilon>0$, there is a $\Lambda>0$, such that when $\lambda \geq \Lambda$,

$$
C_{\lambda}^{l}(f)\left(x_{0}\right) \leq f\left(x_{0}\right) \leq C_{\lambda}^{l}(f)\left(x_{0}\right)+\frac{\epsilon^{2}}{4 \lambda} .
$$

(iii) Suppose $f: \mathbb{R}^{n} \mapsto \mathbb{R}$ is a globally Lipschitz function with Lipschitz constant $L>0$. Then for every $\lambda>0$ and for every $x \in \mathbb{R}^{n}$,

$$
C_{\lambda}^{l}(f)(x) \leq f(x) \leq C_{\lambda}^{l}(f)(x)+\frac{L^{2}}{4 \lambda} .
$$


These results can be generalised for $f$ bounded and uniformly continuous in $\mathbb{R}^{n}$ using the modulus of continuity. We recall from Ref. 16 the definition of modulus of continuity of a function along with some of its properties.

Definition 2.13. Let $f: \mathbb{R}^{n} \mapsto \mathbb{R}$ be a bounded and uniformly continuous function in $\mathbb{R}^{n}$. Then,

$$
\omega_{f}: t \in[0, \infty) \mapsto \omega_{f}(t)=\sup \left\{|f(x)-f(y)|: x, y \in \mathbb{R}^{n} \text { and }|x-y| \leq t\right\}
$$

is called the modulus of continuity of $f$.

Proposition 2.14. Let $f: \mathbb{R}^{n} \mapsto \mathbb{R}$ be a bounded and uniformly continuous function in $\mathbb{R}^{n}$. Then the modulus of continuity $\omega_{f}$ of $f$ satisfies the following properties:

(i) $\omega_{f}(t) \rightarrow \omega(0)=0$, as $t \rightarrow 0$;

(ii) $\omega_{f}$ is non-negative and non-decreasing continuous function on $[0, \infty)$;

(iii) $\omega_{f}$ is subadditive: $\omega_{f}\left(t_{1}+t_{2}\right) \leq \omega_{f}\left(t_{1}\right)+\omega_{f}\left(t_{2}\right)$ for all $t_{1}, t_{2} \geq 0$.

A function $\omega$ defined on $[0, \infty)$ and satisfying (2.16) is called a modulus of continuity. A modulus of continuity $\omega$ can be bounded from above by an affine function (see Lemma 6.1 of Ref. 16), that is, there exist some constants $a>0$ and $b \geq 0$ such that

$$
\omega(t) \leq a t+b \quad(\text { for all } t \geq 0) .
$$

As a result, given $\omega_{f}$, one can define the least concave majorant of $\omega_{f}$, which we denote by $\omega_{f}^{c}$, which is also a modulus of continuity with the property (see Ref. 16)

$$
\frac{1}{2} \omega_{f}^{c}(t) \leq \omega_{f}(t) \leq \omega_{f}^{c}(t) \quad(\text { for all } t \in[0, \infty)) .
$$

The next theorem gives a complete picture of how compensated convex transforms can approximate uniformly continuous functions.

Theorem 2.15. (Approximations for uniformly continuous functions) Let $f$ : $\mathbb{R}^{n} \mapsto \mathbb{R}$ be bounded and uniformly continuous and denote by $\omega_{f}^{c}$ the least concave majorant of the modulus of continuity $\omega_{f}$ of $f$. Assume $a>0, b \geq 0$ are such that $\omega_{f}^{c}(t) \leq a t+b$ for $t \in[0,+\infty)$. Then for every $\lambda>0$,

$$
\begin{aligned}
f(x)-\omega_{f}^{c}\left(\frac{a}{\lambda}+\sqrt{\frac{b}{\lambda}}\right) & \leq C_{\lambda}^{l}(f)(x) \leq f(x) \quad\left(\text { for } x \in \mathbb{R}^{n}\right), \\
f(x) & \leq C_{\lambda}^{u}(f)(x) \leq f(x)+\omega_{f}^{c}\left(\frac{a}{\lambda}+\sqrt{\frac{b}{\lambda}}\right)\left(\text { for } x \in \mathbb{R}^{n}\right) .
\end{aligned}
$$

and

We conclude this section with definitions of types of regularity of a domain and its boundary points. 
Definition 2.16. Let $\Omega \subset \mathbb{R}^{n}$ be a bounded open set with $|\partial \Omega|=0$ (i.e. $\partial \Omega$ has zero n-dimensional measure), and $x_{0} \in \mathbb{R}^{n}$.

(i) The set $\Omega$ satisfies the $\delta$-exterior ball property with $\delta>0$ at a point $x \in \partial \Omega$ if there is an open ball $B\left(x_{0} ; \delta\right) \subset \bar{\Omega}^{c}$ such that $x \in \partial B\left(x_{0} ; \delta\right)$. We call $x$ an exterior $\delta$-regular point of $\partial \Omega$.

(ii) The set $\Omega$ satisfies the $\delta$-interior ball property with $\delta>0$ at a point $x \in \partial \Omega$ if there is an open ball $B\left(x_{0} ; \delta\right) \subset \Omega$ such that $x \in \partial B\left(x_{0} ; \delta\right)$. We call $x$ an interior $\delta$-regular point of $\partial \Omega$.

(iii) If a point $x \in \partial \Omega$ satisfies both the $\delta$-interior ball property and the $\delta$-exterior ball property, we call $x$ a $\delta$-regular point of $\partial \Omega$. A non-empty domain $\Omega \subset \mathbb{R}^{n}$ is called a $\delta$-regular domain (or for short, regular) if there exists a $\delta>0$ such that $\Omega$ is $\delta$-regular at each point of its boundary.

Remark 2.17. These definitions of interior and exterior ball properties have been used extensively in the study of elliptic partial differential equations. ${ }^{19}$ Notice that it can happen that a boundary point of a domain can be both an exterior and interior corner point.

\section{Further analytic and geometric properties of compensated convex transforms}

For a locally bounded function $f: \mathbb{R}^{n} \mapsto \mathbb{R}$, let us consider the upper and the lower semi-continuous closure $\bar{f}$ and $\underline{f}^{23,34}$ defined, respectively, by

$$
\bar{f}(x)=\limsup _{y \rightarrow x} f(y) \quad \text { and } \quad \underline{f}(x)=\liminf _{y \rightarrow x} f(y) .
$$

Then $\bar{f}$ is upper semicontinuous (i.e. $\bar{f}(x) \geq \limsup _{y \rightarrow x} \bar{f}(y)$ ) and $\underline{f}$ is lower semicontinuous (i.e. $\left.\underline{f}(x) \leq \liminf _{y \rightarrow x} \underline{f}(y)\right) \cdot{ }^{23,34}$ Recall also that $\bar{f}$ is the pointwise infimum of the upper semicontinuous functions that are greater than $f$, whereas $f$ is the pointwise supremum of the lower semicontinuous functions that are lower than $f$. It was established in Ref. 48 that if $f$ is lower semicontinuous (respectively, upper semicontinuous) and satisfies Condition (L) (respectively, Condition (U)) in (1.1), then

$$
\lim _{\lambda \rightarrow \infty} C_{\lambda}^{l}(f)(x)=f(x) \quad\left(\text { respectively, } \quad \lim _{\lambda \rightarrow \infty} C_{\lambda}^{u}(f)(x)=f(x)\right) \quad x \in \mathbb{R}^{n} .
$$

Next we characterize the limit of compensated convex transforms for $\lambda \rightarrow \infty$ for more general functions.

Proposition 3.1. If $f: \mathbb{R}^{n} \mapsto \mathbb{R}$ satisfies Condition (L) (respectively, (U)) in (1.1), then

$$
C_{\lambda}^{l}(f)(x)=C_{\lambda}^{l}(\underline{f})(x) \quad\left(\text { respectively, } \quad C_{\lambda}^{u}(f)(x)=C_{\lambda}^{u}(\bar{f})(x)\right) \quad x \in \mathbb{R}^{n} .
$$


Consequently,

$$
\lim _{\lambda \rightarrow \infty} C_{\lambda}^{l}(f)(x)=\underline{f}(x) \quad\left(\text { respectively, } \quad \lim _{\lambda \rightarrow \infty} C_{\lambda}^{u}(f)(x)=\bar{f}(x)\right) \quad x \in \mathbb{R}^{n} .
$$

Note that the convergence result (3.2) follows from properties of Moreau envelopes (see, for instance, Prop. 1.1 of Ref. 5) and the ordering property (2.4).

Theorem 3.2. (Closure and Conversion Properties) Let $E$ be a subset of $\mathbb{R}^{n}, E^{c}$ the complement of $E$ in $\mathbb{R}^{n}$, i.e. $E^{c}=\mathbb{R}^{n} \backslash E$ and $\stackrel{o}{E}$ the interior of $E$. Assume $\lambda>0$. Then

(i) $C_{\lambda}^{u}\left(\chi_{E}\right)(x)+C_{\lambda}^{l}\left(\chi_{E^{c}}\right)(x)=1$ for all $x \in \mathbb{R}^{n}$.

(ii) For the upper transform,

$$
C_{\lambda}^{u}\left(\chi_{E}\right)(x)=C_{\lambda}^{u}\left(\chi_{\bar{E}}\right)(x) \quad \text { for all } x \in \mathbb{R}^{n} .
$$

(iii) For the lower transform, if $\overline{E^{c}}=\mathbb{R}^{n}$, or equivalently, $\stackrel{\circ}{E}=\varnothing$, then

$$
C_{\lambda}^{l}\left(\chi_{E}\right)(x) \equiv 0 \text { for all } x \in \mathbb{R}^{n}
$$

Remark 3.3. Statement (iii) does not hold for the lower transform of a characteristic function in general, though it holds if $\stackrel{o}{E} \neq \varnothing$. If $\stackrel{o}{E}=\varnothing$ and $E$ is dense in $\mathbb{R}^{n}$, for example, if $E$ consists of all points with rational components, then $C_{\lambda}^{l}\left(\chi_{E}\right) \equiv 0$. However, since $\bar{E}=\mathbb{R}^{n}$, we have $C_{\lambda}^{l}\left(\chi_{\bar{E}}\right) \equiv 1$.

The following result characterizes the values of the upper transform of the characteristic function of a non-empty subset $E$ of $\mathbb{R}^{n}$.

Theorem 3.4. (Expansion Theorem) Let $E \subset \mathbb{R}^{n}$ be a non-empty set and let $\lambda>0$ be fixed, then for $x \in \mathbb{R}^{n}$

$$
C_{\lambda}^{u}\left(\chi_{E}\right)(x) \quad \begin{cases}=1 & \text { if } x \in \bar{E} \\ =0 & \text { if } x \in\left(E^{1 / \sqrt{\lambda}}\right)^{c} \\ \in(0,1) & \text { if } x \in E^{1 / \sqrt{\lambda}} \backslash \bar{E} .\end{cases}
$$

It is possible to have a better understanding on how $C_{\lambda}^{u}\left(\chi_{\Omega}\right)(x)$ is defined 'near' the regular points of $\partial \Omega$. For a bounded non-empty set $E \subset \mathbb{R}^{n}$ and a fixed $\lambda>0$, Theorem 3.4 states that the support of $C_{\lambda}^{u}\left(\chi_{E}\right)$ is the set $\overline{E^{1 / \sqrt{\lambda}}}$. We now establish an upper bound for $C_{\lambda}^{u}\left(\chi_{E}\right)$ that vanishes exactly at $x$, with $x \in \partial E^{1 / \sqrt{\lambda}}$. We also show that the expansion operation $C_{\lambda}^{u}\left(\chi_{E}\right)$ of a domain $\Omega$ is smoothly attached to the background.

Proposition 3.5. Let $E \subset \mathbb{R}^{n}$ and $\lambda>0$ be such that $\overline{E^{1 / \sqrt{\lambda}}} \neq \mathbb{R}^{n}$. For all $x \in \partial E^{1 / \sqrt{\lambda}}$

(i) $C_{\lambda}^{u}\left(\chi_{E}\right)$ is bounded above by $C_{\lambda}^{u}\left(\chi_{B^{c}(x ; 1 / \sqrt{\lambda})}\right)$ in $\mathbb{R}^{n}$, where $B^{c}(x ; 1 / \sqrt{\lambda})$ is the complement in $\mathbb{R}^{n}$ of the ball $B(x ; 1 / \sqrt{\lambda})$, i.e. for any $x \in \partial E^{1 / \sqrt{\lambda}}$,

$$
C_{\lambda}^{u}\left(\chi_{E}\right)(y) \leq C_{\lambda}^{u}\left(\chi_{B^{c}(x ; 1 / \sqrt{\lambda})}\right)(y) \quad \text { for all } y \in \mathbb{R}^{n}
$$


and

$$
C_{\lambda}^{u}\left(\chi_{B^{c}(x ; 1 / \sqrt{\lambda})}\right)(x)=0 .
$$

(ii) $C_{\lambda}^{u}\left(\chi_{E}\right)$ is differentiable at $x$ and the gradient of $C_{\lambda}^{u}\left(\chi_{E}\right)$ satisfies $D C_{\lambda}^{u}\left(\chi_{E}\right)(x)=0$.

The following example gives a prototype for the behaviour of the upper and lower transforms near regular points.

Example 3.6. Let $E=\left\{(x, y) \in \mathbb{R}^{2}, x \leq 0\right\}$. According to Definition 2.16, every point on $\partial E$ is a $\delta$-regular point for any $\delta>0$. Let $f(x, y)=\chi_{E}(x, y)$, then for $(x, y) \in \mathbb{R}^{2}$

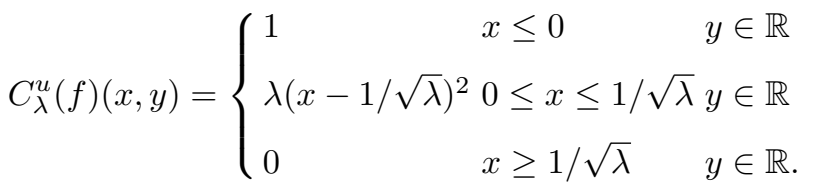

$$
\begin{aligned}
& C_{\lambda}^{l}(f)(x, y)=\left\{\begin{array}{llr}
1 & x \leq-1 / \sqrt{\lambda} & y \in \mathbb{R} \\
1-\lambda(x+1 / \sqrt{\lambda})^{2} & 1 / \sqrt{\lambda} \leq x \leq 0 & y \in \mathbb{R} \\
0 & x \geq 0 & y \in \mathbb{R} .
\end{array}\right.
\end{aligned}
$$

The following theorem extends Example 3.6 to general exterior and interior $\delta$-regular points.

Theorem 3.7. (Regular extension) Suppose $\lambda>0, x_{0} \in \mathbb{R}^{n}$ and $\Omega \subset \mathbb{R}^{n}$ is an open set satisfying $\bar{\Omega} \neq \mathbb{R}^{n}$.

(i) Suppose $\Omega$ satisfies the $\delta$-exterior ball property at $x \in \partial \Omega$ with radius $\delta \geq 1 / \sqrt{\lambda}$. Let $B\left(x_{0} ; \delta\right) \subset(\bar{\Omega})^{c}$ be the ball such that $x \in \partial B\left(x_{0} ; \delta\right)$. Let $\left[x, x_{0}\right]$ be the line segment connecting $x$ and $x_{0}$ and $x_{1} \in\left[x, x_{0}\right]$ be such that $\left|x_{1}-x\right|=1 / \sqrt{\lambda}$. Then for $y \in\left[x, x_{0}\right]$

$$
C_{\lambda}^{u}\left(\chi_{\Omega}\right)(y)= \begin{cases}\lambda\left|y-x_{1}\right|^{2} & y \in\left[x, x_{1}\right] \\ 0 & y \in\left[x_{1}, x_{0}\right] .\end{cases}
$$

(ii) Suppose $\Omega$ satisfies the $\delta$-interior ball property at $x \in \partial \Omega$ with radius $\delta \geq 1 / \sqrt{\lambda}$. Let $B\left(x_{0} ; \delta\right) \subset \Omega$ be the ball such that $x \in \partial B\left(x_{0} ; \delta\right)$. Let $\left[x, x_{0}\right]$ be the line segment connecting $x$ and $x_{0}$ and $x_{1} \in\left[x, x_{0}\right]$ be such that $\left|x_{1}-x\right|=1 / \sqrt{\lambda}$. Then for $y \in\left[x, x_{0}\right]$

$$
C_{\lambda}^{l}\left(\chi_{\Omega}\right)(y)= \begin{cases}1-\lambda|y-x|^{2} & y \in\left[x, x_{1}\right] \\ 1 & y \in\left[x_{1}, x_{0}\right] .\end{cases}
$$

The following theorem is a stability result for compensated convex transforms under curvature perturbation. The result is a simpler version of the stability theorems for ridge and valley transforms in the next section. 
Theorem 3.8. Let $f: \mathbb{R}^{n} \mapsto \mathbb{R}$ be a bounded function and $\lambda_{0}>0$. Suppose the smooth perturbation $g \in C^{1,1}\left(\mathbb{R}^{n}\right)$ satisfies

$$
|D g(x)-D g(y)| \leq \lambda_{0}|x-y| \quad \text { for all } x, y \in \mathbb{R}^{n} .
$$

Then for every $\lambda>\lambda_{0} / 2$ and $x \in \mathbb{R}^{n}$,

$$
\begin{aligned}
& g(x)+C_{\lambda-\frac{\lambda_{0}}{2}}^{l}(f)(x) \leq C_{\lambda}^{l}(f+g)(x) \leq g(x)+C_{\lambda+\frac{\lambda_{0}}{2}}^{l}(f)(x) \\
& g(x)+C_{\lambda+\frac{\lambda_{0}}{2}}^{u}(f)(x) \leq C_{\lambda}^{u}(f+g)(x) \leq g(x)+C_{\lambda-\frac{\lambda_{0}}{2}}^{u}(f)(x) .
\end{aligned}
$$

The following lemma gives an estimate on the points needed to compute the value of the convex envelope of $f_{\lambda}(x)=\lambda|x|^{2}-\chi_{E}(x)$ at the point $x_{0}=0$. The lemma is a special case of the locality property stated in Theorem 3.10(i).

Lemma 3.9. Let $\lambda>0$ and consider a closed subset $E$ of $\mathbb{R}^{n}$ with $E \neq \mathbb{R}^{n}$. Let $f_{\lambda}(x)=\lambda|x|^{2}-\chi_{E}(x)$. By Proposition 2.1(i), there exist $\left(\tau_{i}, x_{i}\right) \in \mathbb{R} \times \mathbb{R}^{n}$, $i=1, \ldots, n+1$, such that

$$
\operatorname{co}\left[f_{\lambda}\right](0)=\sum_{i=1}^{n+1} \tau_{i} f_{\lambda}\left(x_{i}\right) \quad \text { with } \tau_{i} \geq 0, \quad \sum_{i=1}^{n+1} \tau_{i}=1, \quad \text { and } \sum_{i=1}^{n+1} \tau_{i} x_{i}=0 .
$$

Then,

$$
\left|x_{i}\right| \leq A(\lambda) \text { with } A(\lambda)=(1+\sqrt{2}) / \sqrt{\lambda}
$$

and

$$
-1 \leq \operatorname{co}\left[f_{\lambda}\right](0) \leq 0 .
$$

Next we state a locality and density property for the upper and lower transforms applied to general bounded functions. The proof of locality given here exploits the characterization (1.6) of the transforms as critical mixed Moreau envelopes. Without loss of generality, by Proposition 3.1, we can assume the functions to be lower (respectively, upper) semicontinuous. For the locality properties of more general functions, the reader is referred to Theorem 2.4 of Ref. 48 . The density property can be viewed as a tight approximation property for general bounded functions.

Theorem 3.10. (The locality and density properties for bounded functions) Suppose $f: \mathbb{R}^{n} \mapsto \mathbb{R}$ is bounded, satisfying $|f(x)| \leq M$ for some $M>0$ and for all $x \in \mathbb{R}^{n}$. Let $\lambda>0$ and $x_{0} \in \mathbb{R}^{n}$.

(i) The following locality properties hold:

$$
\begin{aligned}
& C_{\lambda}^{l}(f)\left(x_{0}\right)=\operatorname{co}_{\bar{B}\left(x_{0} ; R_{\lambda, M}\right)}\left[f+\lambda\left|(\cdot)-x_{0}\right|^{2}\right]\left(x_{0}\right), \\
& C_{\lambda}^{u}(f)\left(x_{0}\right)=-\operatorname{co}_{\bar{B}\left(x_{0} ; R_{\lambda, M}\right)}\left[\lambda\left|(\cdot)-x_{0}\right|^{2}-f\right]\left(x_{0}\right)
\end{aligned}
$$

with $R_{\lambda, M} \leq 2 \sqrt{2} \sqrt{\frac{M}{\lambda}}$, and $\operatorname{co}_{\bar{B}\left(x_{0} ; R_{\lambda, M}\right)}[g]\left(x_{0}\right)$ the value of the local convex envelope of $g$ at $x_{0}$ in $\bar{B}\left(x_{0} ; R_{\lambda, M}\right)$ defined according to Definition 2.2. Consequently,

$$
C_{\lambda}^{l}(f)\left(x_{0}\right)=C_{\lambda}^{l}\left(f_{x_{0}}\right)\left(x_{0}\right), \quad C_{\lambda}^{u}(f)\left(x_{0}\right)=C_{\lambda}^{u}\left(f_{x_{0}}\right)\left(x_{0}\right)
$$


where

$$
f_{x_{0}}(x):=f(x) \chi_{\bar{B}\left(x_{0} ; R(\lambda, M)\right)}(x), \quad x \in \mathbb{R}^{n} .
$$

(ii) The following density property holds:

(a) If

$$
C_{\lambda}^{l}(f)\left(x_{0}\right)<\underline{f}\left(x_{0}\right)
$$

there are $x_{i} \in \bar{B}\left(x_{0} ; R_{\lambda, M}\right)$, with $x_{i} \neq x_{0}$, and $\lambda_{i} \geq 0$ for $i=1, \ldots, n+1$, satisfying $\sum_{i=1}^{n+1} \lambda_{i}=1$ and $\sum_{i=1}^{n+1} \lambda_{i} x_{i}=x_{0}$, such that

$$
C_{\lambda}^{l}(f)\left(x_{i}\right)=\underline{f}\left(x_{i}\right) \quad \text { for } i=1, \ldots, n+1 .
$$

(b) If

$$
C_{\lambda}^{u}(f)\left(x_{0}\right)>\bar{f}\left(x_{0}\right),
$$

there are $z_{i} \in \bar{B}\left(x_{0} ; R_{\lambda, M}\right)$, with $z_{i} \neq x_{0}$, and $\tau_{i} \geq 0$ for $i=1, \ldots, n+1$, satisfying $\sum_{i=1}^{n+1} \tau_{i}=1$ and $\sum_{i=1}^{n+1} \tau_{i} z_{i}=x_{0}$, such that

$$
C_{\lambda}^{l}(f)\left(z_{i}\right)=\bar{f}\left(z_{i}\right) \quad \text { for } i=1, \ldots, n+1 .
$$

If $f$ is bounded and continuous, all statements in Theorem 3.10(ii) for $f$ and $\bar{f}$ hold also for $f$, given that for continuous functions one has: $f=\bar{f}=f$. Using the approximation properties stated in Theorem 2.12, it is possible to establish sharper locality and density properties if $f$ has more smoothness.

\section{Remark 3.11.}

(a) Part (i) of Theorem 3.10 simply says that the value of the lower (respectively, upper) transforms for a bounded function at a point is determined by the values of the function in its $R_{\lambda, M}$ neighbourhood. Therefore when $\lambda>0$ is large, the neighbourhood will be very small. If $f$ is globally Lipschitz, our result is a special case of Lemma 3.5.7 at pag. 72 of Ref. 12.

(b) For the upper transform $C_{\lambda}^{u}\left(\chi_{E}\right)$ of the characteristic function of a set $E \subset \mathbb{R}^{n}$, Lemma 3.9 provides a slightly better estimate $R_{\lambda, M} \leq(1+\sqrt{2}) / \sqrt{\lambda}$. When $n=1$, a direct calculation of the supporting points $x_{i}$ shows that $\left|x_{i}\right| \leq 1 / \sqrt{\lambda}$. This is also the case for $n>1$ if $f_{\lambda}(x)=\lambda|x|^{2}-\chi_{E}(x)$ is differentiable at $x_{i}$.

(c) Part (ii) of Theorem 3.10 says that the sets of points at which the compensated convex transforms equal the original function satisfy a density property. Recall first that, in general,

$$
C_{\lambda}^{l}(f) \leq \underline{f} \leq f \leq \bar{f} \leq C_{\lambda}^{u}(f) .
$$

Suppose now $f$ satisfies the assumptions of Theorem 3.10 and define the following sets

$$
\begin{aligned}
& T_{u}(f, \lambda)=\left\{x \in \mathbb{R}^{n}: C_{\lambda}^{u}(f)(x)=\bar{f}(x)\right\} \quad \text { and } \\
& T_{l}(f, \lambda)=\left\{x \in \mathbb{R}^{n}: C_{\lambda}^{l}(f)(x)=\underline{f}(x)\right\} .
\end{aligned}
$$


We can call $T_{u}(f, \lambda)$ and $T_{l}(f, \lambda)$ the upper and lower touching sets of the compensated convex transforms. Then the closed $R_{\lambda, M}$-neighbourhoods of $T_{u}(f, \lambda)$ and $T_{l}(f, \lambda)$ both cover $\mathbb{R}^{n}$.

(d) Our density property can be viewed as a convex covering density in the sense that for any point $x_{0}$, the point $x_{0}$ is contained in both the local convex hulls co $\left[T_{l}(f, \lambda) \cap \bar{B}\left(x_{0} ; R_{\lambda, M}\right)\right]$ and co $\left[T_{u}(f, \lambda) \cap \bar{B}\left(x_{0} ; R_{\lambda, M}\right)\right]$. If we call $R_{\lambda, M}$ the radius of density for $T_{u}(f, \lambda)$ and $T_{l}(f, \lambda)$, Part (ii) also implies that the radius of density shrinks to zero as $\lambda \rightarrow+\infty$.

(e) If $f$ is bounded and continuous, $T_{l}(f, \lambda)$ and respectively, $T_{u}(f, \lambda)$ are exactly the sets of points at which $f$ are $\lambda$-semiconvex and respectively, $\lambda$-semiconcave, i.e. the points $x_{0}$ where

$$
f(x) \geq f\left(x_{0}\right)+\ell(x)-\lambda\left|x-x_{0}\right|^{2} \quad \text { for all } x \in \mathbb{R}^{n}
$$

(respectively, $\quad f(x) \leq f\left(x_{0}\right)+\ell(x)+\lambda\left|x-x_{0}\right|^{2} \quad$ for all $x \in \mathbb{R}^{n}$ ),

with $\ell$ an affine function satisfying $\ell\left(x_{0}\right)=0$ and $f$ meeting Condition (L) (respectively Condition (U).

$(f)$ The density property provides also another angle from which to view our tight approximation property in Ref. 48, where we proved that if $f$ satisfies condition (B) in a neighbourhood of $x_{0}, f$ is $C^{1,1}$, then $C_{\lambda}^{l}(f)\left(x_{0}\right)=C_{\lambda}^{u}(f)\left(x_{0}\right)=f\left(x_{0}\right)$ as long as $\lambda>0$ is sufficiently large. If we further assume that $f$ is bounded and continuous, the density property says that the graphs of the lower and upper transforms touch that of $f$ on sets whose density can be measured.

The following result is a special case of Theorem 3.5.3 at pag. 70 of Ref. 12 .

Theorem 3.12. Suppose $f: \mathbb{R}^{n} \mapsto \mathbb{R}$ is a globally Lipschitz function with Lipschitz constant $L>0$. Then for $\lambda>0, C_{\lambda}^{l}(f)$ and $C_{\lambda}^{u}(f)$ are both globally Lipschitz functions satisfying for all $x, y \in \mathbb{R}^{n}$

$$
\left|C_{\lambda}^{l}(f)(x)-C_{\lambda}^{l}(f)(y)\right| \leq L|x-y| ; \quad\left|C_{\lambda}^{u}(f)(x)-C_{\lambda}^{u}(f)(y)\right| \leq L|x-y| .
$$

Next we give an estimate of the Lipschitz constants for compensated convex transforms applied to bounded functions and use Theorem 2.12(iii) to examine error bounds for the mixed transforms approaching upper and lower transforms.

Theorem 3.13. Suppose $f: \mathbb{R}^{n} \mapsto \mathbb{R}$ is bounded, that is, $|f(x)| \leq M$ for some $M>0$ and for $x \in \mathbb{R}^{n}$. Then

(i) Both $C_{\lambda}^{l}(f)$ and $C_{\lambda}^{u}(f)$ are Lipschitz functions with Lipschitz constants bounded by $8 \sqrt{M \lambda}$, that is, for all $x, y \in \mathbb{R}^{n}$,

$$
\left|C_{\lambda}^{l}(f)(x)-C_{\lambda}^{l}(f)(y)\right| \leq 8 \sqrt{M \lambda} \quad\left|C_{\lambda}^{u}(f)(x)-C_{\lambda}^{u}(f)(y)\right| \leq 8 \sqrt{M \lambda}|x-y| .
$$

(ii) For $\lambda>0$ and $\tau>0$, the mixed transforms $C_{\tau}^{u}\left(C_{\lambda}^{l}(f)(x)\right.$ and $C_{\tau}^{l}\left(C_{\lambda}^{u}(f)(x)\right.$ are 
Lipschitz functions with Lipschitz constants bounded by $8 \sqrt{M \lambda}$ and for $x \in \mathbb{R}^{n}$

$$
\begin{aligned}
& 0 \leq C_{\lambda}^{u}(f)(x)-C_{\tau}^{l}\left(C_{\lambda}^{u}(f)(x) \leq \frac{16 M \lambda}{\tau} ;\right. \\
& 0 \leq C_{\tau}^{u}\left(C_{\lambda}^{l}(f)(x)-C_{\lambda}^{l}(f)(x) \leq \frac{16 M \lambda}{\tau} .\right.
\end{aligned}
$$

In fact, the mixed transforms above are bounded $C^{1,1}$ functions. We will discuss this elsewhere.

\section{Ridge, valley and edge transforms}

In this section we introduce the notions of ridge, valley and edge transforms and study their properties. These are basic operations for extracting geometric singularities.

Definition 4.1. For a function $f: \mathbb{R}^{n} \mapsto \mathbb{R}$ satisfying (B) in (1.1), assume $A>0$ the constant entering the conditions (1.1), we define the translation invariant ridge $R_{\lambda}(f)$, valley $V_{\lambda}(f)$ and edge $E_{\lambda}(f)$ transform for $f$ of scale $\lambda>A$ by, respectively,

$$
\begin{aligned}
& R_{\lambda}(f)=f-C_{\lambda}^{l}(f) ; \quad V_{\lambda}(f)=f-C_{\lambda}^{u}(f) ; \\
& E_{\lambda}(f)=R_{\lambda}(f)-V_{\lambda}(f)=C_{\lambda}^{u}(f)-C_{\lambda}^{l}(f) .
\end{aligned}
$$

If $f$ is of sub-quadratic growth, that is, $|f(x)| \leq A\left(1+|x|^{\alpha}\right)$ with $0 \leq \alpha<2$, in particular $f$ can be a bounded function, the requirement for $\lambda$ in Definition 4.1 is simply $\lambda>0$.

\section{Remark 4.2.}

(a) Since the original function $f$ is directly involved in the definitions of our ridge, valley and edge transforms, these transforms are not Hausdorff stable if we consider a dense sampling of the original function. In the following Section, however, we will introduce our stable versions of ridge and valley transforms which are based on these basic transforms.

(b) The ridge transform $R_{\lambda}(f)=f-C_{\lambda}^{l}(f)$ and the valley transform $V_{\lambda}(f)=f-$ $C_{\lambda}^{u}(f)$ are non-negative and non-positive, respectively, because of the ordering property (2.4) of the compensated convex transforms. As a result, the geometry of ridges and valleys is better reflected and the algebraic properties stated in Proposition 4.4 below hold. However, in practice, we usually consider $-V_{\lambda}(f)$ to make the resulting function non-negative.

(c) When $f$ is a bounded and continuous function, say, $|f(x)| \leq M$ for some $M>0$, our density property (Theorem 3.10(ii)) implies that the set on which $R_{\lambda}(f)(x)=0$ (respectively, $V_{\lambda}(f)(x)=0$ ) has the property that its closed $R_{\lambda, M}$-neighbourhood is the whole space $\mathbb{R}^{n}$. However, without further knowledge of the function, we cannot say that the supports of $R_{\lambda}(f)$ and $V_{\lambda}(f)$ are 'thin' sets. 
The following result shows that our ridge and valley transforms are both invariant with respect to translation, and scale covariant.

\section{Proposition 4.3.}

(i) The transforms $R_{\lambda}(f)$ and $V_{\lambda}(f)$ are invariant with respect to translation, in the sense that

$$
R_{\lambda}(f+\ell)=R_{\lambda}(f) \quad \text { and } \quad V_{\lambda}(f+\ell)=V_{\lambda}(f)
$$

for all affine functions $\ell \in \operatorname{Aff}\left(\mathbb{R}^{n}\right)$. Consequently, the edge transform $E_{\lambda}(f)$ is also invariant with respect to translation.

(ii) The transforms $R_{\lambda}(f)$ and $V_{\lambda}(f)$ are scale covariant in the sense that

$$
R_{\lambda}(\alpha f)=\alpha R_{\lambda / \alpha}(f) \quad \text { and } \quad V_{\lambda}(\alpha f)=\alpha V_{\lambda / \alpha}(f)
$$

for all $\alpha>0$. Consequently, the edge transform $E_{\lambda}(f)$ is also scale covariant.

The numerical experiment depicted in Figure 2 illustrates the property defined by equation (4.2) for the edge transform $E_{\lambda}$. We use a $256 \times 256$ binary image of a Chinese character and denote by $\chi(i, j)$ its characteristic function. Since the format uint8 can only display values between 0 and 255 , we consider the affine function $\ell(i, j)=i-1$ and define $f(i, j)=3 \chi(i, j)+\ell(i, j)$ for $1 \leq i, j \leq 256$. Observe that, for instance, Canny edge detector is not invariant with respect to translation.

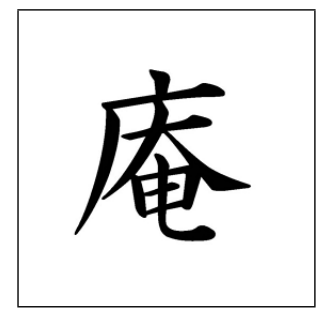

$(a)$

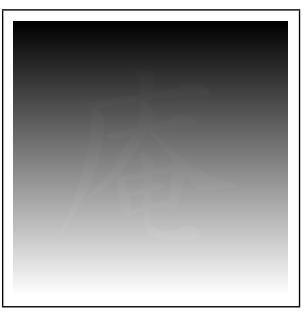

(b)

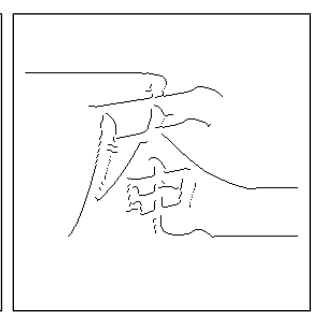

$(c)$

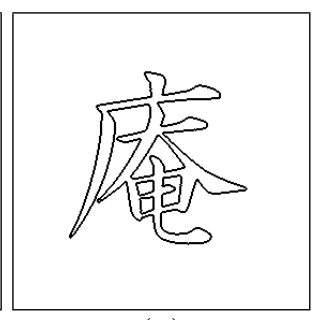

$(d)$

Fig. 2. (a) A binary image $\chi$ of a Chinese character; (b) Image $3 \chi+\ell$, i.e. the scaled characteristic function of the character plus a simple affine function; (c) Edges extracted by Canny edge detector; $(d)$ Edges extracted by the edge transform $E_{\lambda}(f)$ with $\lambda=1$ after thresholding.

The ridge and valley transforms satisfy also the following properties which, for simplicity, are stated for bounded functions.

Proposition 4.4. Suppose $f: \mathbb{R}^{n} \mapsto \mathbb{R}$ is bounded. Then for every $\lambda>0$ and $\tau>0$, we have
(i) $R_{\lambda}(f)=-V_{\lambda}(-f)$
and $V_{\lambda}(f)=-R_{\lambda}(-f)$;
(ii) $R_{\tau+\lambda}(f) \leq R_{\tau}\left(R_{\lambda}(f)\right) \leq R_{\lambda}(f)$ and $V_{\lambda}(f) \leq V_{\tau}\left(V_{\lambda}(f)\right) \leq V_{\tau+\lambda}(f)$. 
Remark 4.5. For many one dimensional functions, we can verify that $R_{\tau}\left(R_{\lambda}(f)\right)=$ $R_{\tau+\lambda}(f)$. However, we suspect that the equality does not hold in general.

The following theorem provides estimates for the stability of our feature detection transforms under curvature perturbations.

Theorem 4.6. The transforms $R_{\lambda}(f), V_{\lambda}(f)$ and $E_{\lambda}(f)$ are all stable under curvature perturbations in the sense that for any $g \in C^{1,1}\left(\mathbb{R}^{n}\right)$ satisfying $\mid D g(x)-$ $D g(y)|\leq \epsilon| x-y \mid$, if $\lambda>\epsilon$ then

$$
\begin{aligned}
& R_{\lambda+\epsilon}(f) \leq R_{\lambda}(f+g) \leq R_{\lambda-\epsilon}(f) ; \quad V_{\lambda-\epsilon}(f) \leq V_{\lambda}(f+g) \leq V_{\lambda+\epsilon}(f) ; \\
& E_{\lambda+\epsilon}(f) \leq E_{\lambda}(f+g) \leq E_{\lambda-\epsilon}(f) .
\end{aligned}
$$

Remark 4.7. Of course when the perturbation $g$ belongs to $C^{2}\left(\mathbb{R}^{n}\right)$ with $\left|D^{2} g(x)\right| \leq \epsilon$, our requirement for $g$ in Theorem 4.6 is satisfied. However, the slightly weaker assumption for $g$ (i.e. $g \in C^{1,1}$ ) is not only a technical improvement. It is known that even if a function $f$ is $C^{\infty}$, in general the best regularity we can expect for the convex envelope co $[f]$ is $C^{1,1} \cdot{ }^{27}$

The numerical experiment displayed in Figure 3 illustrates the stability of our edge transform under curvature perturbations. The performance of the Canny edge detector is also shown.

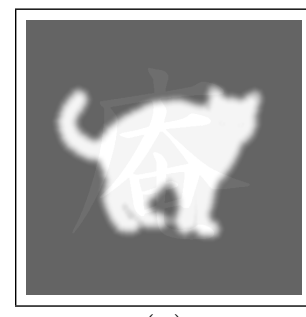

$(a)$

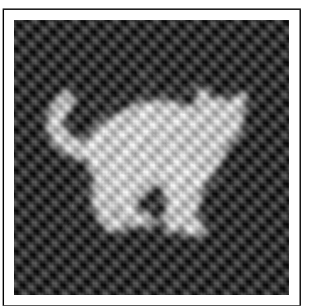

(b)

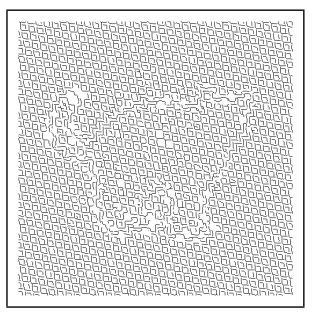

$(c)$

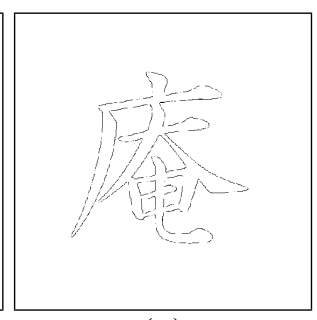

$(d)$

Fig. 3. (a) A scaled binary image of a Chinese character plus a smoothed cat image; (b) The image is perturbed by a trigonometric function; $(c)$ Edges extracted by Canny edge detector; $(d)$ Edges extracted by the edge transform $E_{\lambda}(f)$ after thresholding.

The geometric structure of the Ridge, Valley and Edge Transforms is made precise in the following Theorem.

Theorem 4.8. (Geometric characterization for ridge, valley and edge transforms) Let $\Omega \subset \mathbb{R}^{n}$ be a non-empty open regular set such that $\bar{\Omega} \neq \mathbb{R}^{n}$ and $\Gamma \subset \partial \Omega$. Let $\lambda>0$, then 
(i) The ridge and valley transforms $R_{\lambda}\left(\chi_{\bar{\Omega}}\right)$ and $V_{\lambda}\left(\chi_{\bar{\Omega}}\right)$ satisfy, respectively, for $x \in \mathbb{R}^{n}$

$$
\begin{gathered}
R_{\lambda}\left(\chi_{\Omega \cup \Gamma}\right)(x) \begin{cases}=0 & x \in\left(\Omega^{c} \backslash \Gamma\right) \cup \Omega \backslash\left(\Omega^{c}\right)^{1 / \sqrt{\lambda}} \\
\in(0,1) & x \in\left(\Omega^{c}\right)^{1 / \sqrt{\lambda}} \backslash \Omega^{c} \\
1 & x \in \Gamma .\end{cases} \\
V_{\lambda}\left(\chi_{\Omega \cup \Gamma}\right)(x) \begin{cases}=0 & x \in\left(\Omega^{1 / \sqrt{\lambda}}\right)^{c} \cup(\bar{\Omega} \backslash \Gamma) \\
\in(-1,0) & x \in \Omega^{1 / \sqrt{\lambda}} \backslash \bar{\Omega} \\
=-1 & x \in \Gamma .\end{cases}
\end{gathered}
$$

Furthermore both $R_{\lambda}\left(\chi_{\Omega \cup \Gamma}\right)$ and $V_{\lambda}\left(\chi_{\Omega \cup \Gamma}\right)$ are continuous in $\mathbb{R}^{n} \backslash \partial \Omega$.

(ii) The edge transform $E_{\lambda}\left(\chi_{\Omega \cup \Gamma}\right)$ satisfies, for $x \in \mathbb{R}^{n}$

$$
E_{\lambda}\left(\chi_{\Omega \cup \Gamma}\right)(x) \begin{cases}=0 & x \in\left(\Omega^{1 / \sqrt{\lambda}}\right)^{c} \cup \Omega \backslash\left(\Omega^{c}\right)^{1 / \sqrt{\lambda}} \\ \in(0,1) & x \in \Omega^{1 / \sqrt{\lambda}} \backslash \bar{\Omega} \cup\left(\Omega^{c}\right)^{1 / \sqrt{\lambda}} \backslash \Omega^{c} \\ =1 & x \in \partial \Omega .\end{cases}
$$

Furthermore, $E_{\lambda}\left(\chi_{\Omega \cup \Gamma}\right)$ is continuous in $\mathbb{R}^{n}$ and, for $x \in \mathbb{R}^{n}$

$$
\lim _{\lambda \rightarrow+\infty} E_{\lambda}\left(\chi_{\Omega \cup \Gamma}\right)(x)=\chi_{\partial \Omega}(x) .
$$

The following Proposition shows instead that for points with different smoothness properties, the rate of approximations of upper and lower transforms are different.

Proposition 4.9. Suppose $f: \mathbb{R}^{n} \mapsto \mathbb{R}$ maps bounded sets to bounded sets.

(i) If $f$ is lower semicontinuous and satisfies $(\boldsymbol{L})$ and $f$ is $C^{\alpha}$ at $x_{0}$ for $0<\alpha<2$, then

$$
\limsup _{\lambda \rightarrow \infty} \lambda^{\alpha /(2-\alpha)} R_{\lambda}(f)\left(x_{0}\right) \leq L^{2 /(2-\alpha)}\left(\frac{\alpha}{2}\right)^{\alpha /(2-\alpha)}\left(1-\frac{\alpha}{2}\right) .
$$

(ii) If $f$ is upper semicontinuous and satisfies (U) and $f$ is $C^{\alpha}$ at $x_{0}$ for $0<\alpha<2$, then

$$
\liminf _{\lambda \rightarrow \infty} \lambda^{\alpha /(2-\alpha)} V_{\lambda}(f)\left(x_{0}\right) \geq-L^{2 /(2-\alpha)}\left(\frac{\alpha}{2}\right)^{\alpha /(2-\alpha)}\left(1-\frac{\alpha}{2}\right) .
$$

Proposition 4.10. Suppose $f: \mathbb{R}^{n} \mapsto \mathbb{R}$ is a Lipschitz function with Lipschitz constant $L$. Then for every $x \in \mathbb{R}^{n}$ and for all $\lambda>0$,

$$
\lambda R_{\lambda}(f)(x) \leq \frac{L^{2}}{4} \quad \text { and } \quad \lambda V_{\lambda}(f)(x) \geq-\frac{L^{2}}{4} .
$$

Furthermore, for almost every $x \in \Omega$,

$$
\lim _{\lambda \rightarrow \infty} \lambda R_{\lambda}(f)(x)=0 \quad \text { and } \quad \lim _{\lambda \rightarrow \infty} \lambda V_{\lambda}(f)(x)=0 .
$$




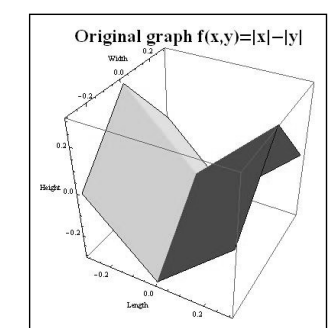

(a)

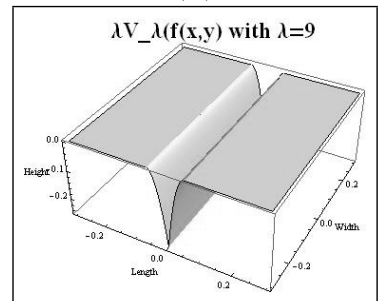

$(d)$

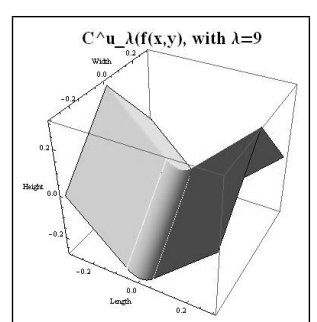

(b)

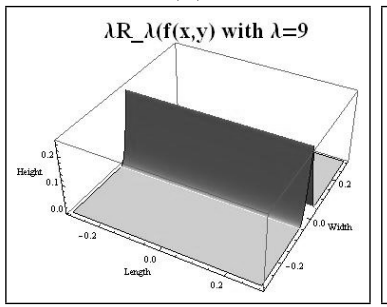

$(e)$

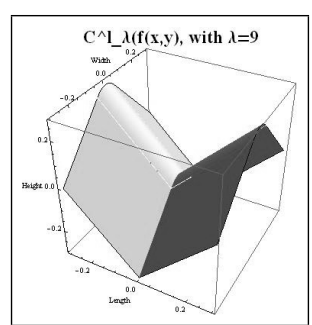

(c)

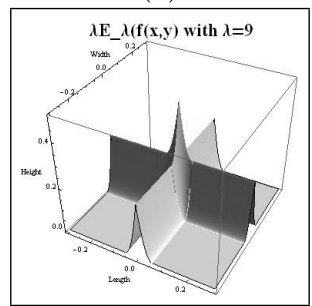

$(f)$

Fig. 4. Graph of the function: (a) $f(x, y)=|x|-|y| ;(b) C_{\lambda}^{u}(f) ;(c) C_{\lambda}^{l}(f) ;(e) \lambda V_{\lambda}(f) ;(f) \lambda R_{\lambda}(f)$; (g) $\lambda E_{\lambda}(f)$ for $\lambda=9$. Observe that the height of the scaled Ridge and Valley transform is less that $1 / 4$ in agreement with Proposition 4.10 .

Figure 4 displays for the special case of $f(x, y)=|x|-|y|$, the graph of its upper and lower transform, the scaled ridge, valley and the edge transform $\lambda V_{\lambda}(f)$, $\lambda R_{\lambda}(f)$, and $\lambda E_{\lambda}(f)$, respectively, for $\lambda=9$.

We conclude this Section by examining singularities of continuous piecewise affine functions defined in $\mathbb{R}^{n}$. Let $f: \mathbb{R}^{n} \mapsto \mathbb{R}$ be a continuous piecewise affine function, that is (see Ref. 21), $f$ is continuous in $\mathbb{R}^{n}$ and there exists a finite collection of closed convex polyhedra with non-empty and mutually disjoint interiors whose union covers the whole space $\mathbb{R}^{n}$ (polyhedral partition) such that the restriction of $f$ to each of the polyhedron of the partition coincides with the restriction therein of an affine function. Clearly, in this case, $f$ is a Lipschitz function and $f$ can be non-differentiable on boundaries of these polyhedra. The following example illustrates such behaviour in a special case.

Example 4.11. Suppose $x_{0} \in \mathbb{R}^{n}$ is a relative interior point of an $n$-1-dimensional polytope so that $f(x)=\min \left\{\ell_{1}(x), \ell_{2}(x)\right\}$ or $f(x)=\max \left\{\ell_{1}(x), \ell_{2}(x)\right\}$ near $x_{0}$, with $\ell_{1}$ and $\ell_{2}$ two affine functions. We consider the case where $f(x)=$ $\min \left\{\ell_{1}(x), \ell_{2}(x)\right\}$ near $x_{0}$ first and calculate the ridge transform.

Proof: Without loss of generality we may assume $x_{0}=0, \ell_{1}(x)=a_{1} \cdot x+b$ and $\ell_{2}(x)=a_{2} \cdot x+b$ with $a_{1} \neq a_{2}$. Let $a=\left(a_{1}-a_{2}\right) / 2$. Since the ridge transform is affine invariant, we may subtract $\ell(x)=\left(a_{1}+a_{2}\right) \cdot x / 2+b$ from $f(x)$ so that $F(x):=f(x)-\ell(x)=\min \{a \cdot x,-a \cdot x\}$ near 0 . Let $g(x)=\min \{a \cdot x,-a \cdot x\}$. If 
we define $E=\operatorname{span}[a]$ and let $E^{\perp}$ be the orthogonal complement of $E$. The lower transform of $g$ is, for $x \in \mathbb{R}^{n}$,

$$
C_{\lambda}^{l}(g)(x)= \begin{cases}\frac{|a|^{2}}{4 \lambda}-\lambda\left|P_{E}(x)\right|^{2}+\left|P_{E^{\perp}}(x)\right|^{2} & \left|P_{E}(x)\right| \leq \frac{|a|}{2 \lambda} \\ g(x) & \left|P_{E}(x)\right| \geq \frac{|a|}{2 \lambda},\end{cases}
$$

where $\left|P_{E}(x)\right|^{2}$ and $\left|P_{E^{\perp}}(x)\right|^{2}$ are orthogonal projections of $x$ to $E$ and $E^{\perp}$ respectively. By the locality property of lower transforms (see Theorem 2.4 of Ref. 48) we have, for large $\lambda>0, C_{\lambda}^{l}(F)(x)=C_{\lambda}^{l}(g)(x)$ near 0 and

$$
R_{\lambda}(f)(0)=R_{\lambda}(F)(0)=R_{\lambda}(g)(0)=\frac{|a|^{2}}{4 \lambda}=\frac{\left|a_{1}-a_{2}\right|^{2}}{16 \lambda} .
$$

Thus $\lambda R_{\lambda}(f)(0)=\left|a_{1}-a_{2}\right|^{2} / 16$. If we compare this with our estimate in Proposition 4.9, we have $L=\max \left\{\left|a_{1}\right|,\left|a_{2}\right|\right\}$ and $\lambda R_{\lambda}(f)(0) \leq \max \left\{\left|a_{1}\right|^{2},\left|a_{2}\right|^{2}\right\} / 4$.

If $f(x)=\max \left\{\ell_{1}(x), \ell_{2}(x)\right\}$ with $\ell_{1}(x)=a_{1} \cdot x+b$ and $\ell_{2}(x)=a_{2} \cdot x+b$, we apply the formula $V_{\lambda}(f)=-R_{\lambda}(-f)$ given by Proposition 4.4. Note that $-f(x)=$ $\min \left\{-\ell_{1}(x),-\ell_{2}(x)\right\}$ so that

$$
R_{\lambda}(-f)(0)=\frac{\left|a_{1}-a_{2}\right|^{2}}{16 \lambda} \text { hence } V_{\lambda}(f)(0)=-\frac{\left|a_{1}-a_{2}\right|^{2}}{16 \lambda} .
$$

Note that if $x_{0}$ is an interior point of an $n$-dimensional convex polyhedron on which $f(x)$ is affine. Then for sufficiently large $\lambda>0, E_{\lambda}(f)\left(x_{0}\right)=0$ as $f$ is locally $C^{1,1}$ near $x_{0}$ (see Theorem 2.3(iv) of Ref. 48).

\section{The Hausdorff stable ridge transform}

We introduce first a distance based function $D_{\lambda}^{2}(x ; E)$ which will serve two purposes: (i) to prove the Hausdorff-Lipschitz continuity of the upper transform $C_{\lambda}^{u}\left(\chi_{E}\right)$ and (ii) to define a Hausdorff-stable operator that will permit the detection of exterior corners of domains and of intersections of surfaces and curves.

Definition 5.1. Let $E \subset \mathbb{R}^{n}$ be a non-empty closed set with $E \neq \mathbb{R}^{n}$ and denote by $\operatorname{dist}(x ; E)=\inf \{y \in E,|y-x|\}$ the Euclidean distance function to $E$ in $\mathbb{R}^{n}$, where $|\cdot|$ is the Euclidean norm in $\mathbb{R}^{n}$. For a given $\lambda>0$, we define

$$
D_{\lambda}(x ; E):=\max \{0,1-\sqrt{\lambda} \operatorname{dist}(x ; E)\}, \quad x \in \mathbb{R}^{n} .
$$

In the following, we will mainly consider $D_{\lambda}^{2}(x ; E)$. Clearly, we have $0 \leq$ $D_{\lambda}^{2}(x ; E) \leq 1$ in $\mathbb{R}^{n}$ with

$$
D_{\lambda}^{2}(x ; E) \begin{cases}=1, & \text { if } x \in E, \\ =0, & \text { if } \operatorname{dist}(x ; E) \geq \frac{1}{\sqrt{\lambda}}, \\ \in(0,1), & \text { if } 0<\operatorname{dist}(x ; E)<\frac{1}{\sqrt{\lambda}} .\end{cases}
$$


The following properties for the function $D_{\lambda}^{2}(x ; E)$ are easy to verify and will be used later.

Proposition 5.2. Suppose $E, F \subset \mathbb{R}^{n}$ be two non-empty closed sets, then (i) if $E \subset F$,

$$
D_{\lambda}^{2}(x ; E) \leq D_{\lambda}^{2}(x ; F), \quad x \in \mathbb{R}^{n} ;
$$

(ii) if $E \cap \bar{B}(x ; 1 / \sqrt{\lambda}) \neq \varnothing$, then

$$
D_{\lambda}^{2}(x ; E)=D_{\lambda}^{2}(x ; E \cap \bar{B}(x ; 1 / \sqrt{\lambda})), \quad x \in \mathbb{R}^{n} .
$$

The following identity justifies the use of $D_{\lambda}^{2}(x ; E)$.

Proposition 5.3. Let $E \subset \mathbb{R}^{n}$ be a non-empty closed set. Let $\lambda>0$. Then

$$
C_{\lambda}^{u}\left(\chi_{E}\right)(x)=C_{\lambda}^{u}\left(D_{\lambda}^{2}(\cdot ; E)\right)(x), \quad x \in \mathbb{R}^{n} .
$$

Lemma 5.4. Let $E, F$ be non-empty compact subsets of $\mathbb{R}^{n}$. Assume $\lambda>0$. Then, the function $D_{\lambda}^{2}(x ; E)$ is Hausdorff-Lipschitz in the sense that

$$
\left|D_{\lambda}^{2}(x ; E)-D_{\lambda}^{2}(x ; F)\right| \leq 2 \sqrt{\lambda} \operatorname{dist}_{\mathcal{H}}(E, F) \quad\left(\text { for } x \in \mathbb{R}^{n}\right) .
$$

Next we state the Hausdorff-Lipschitz continuity of the upper transform $C_{\lambda}^{u}\left(\chi_{E}\right)$ of the characteristic function of a non-empty compact subset $E \subset \mathbb{R}^{n}$.

Theorem 5.5. (Hausdorff-Lipschitz continuity) Let E, F be non-empty compact subsets of $\mathbb{R}^{n}$. Assume $\lambda>0$. Then

$$
\left|C_{\lambda}^{u}\left(\chi_{E}\right)(x)-C_{\lambda}^{u}\left(\chi_{F}\right)(x)\right| \leq 2 \sqrt{\lambda} \operatorname{dist}_{\mathcal{H}}(E, F) \quad\left(\text { for } x \in \mathbb{R}^{n}\right) .
$$

Remark 5.6. If we apply our locality property for bounded functions (Theorem 3.10 ), we see that for any non-empty closed set $E \subset \mathbb{R}^{n}$,

$$
C_{\lambda}^{u}\left(\chi_{E}\right)(x)=C_{\lambda}^{u}\left(\chi_{E \cap \bar{B}(x ; R(\lambda))}\right)(x), \quad x \in \mathbb{R}^{n},
$$

where $0<R(\lambda) \leq(1+\sqrt{2}) / \sqrt{\lambda}$. Here we define $\chi_{\varnothing} \equiv 0$. This observation and the Hausdorff-Lipschitz continuity are two useful tools to design and analyse stable singularity detector operators, such as, our Stable Ridge Transform (see definition below) and other stable operators.

As an immediate consequence of the preceding Theorem 5.5, we obtain

Corollary 5.7. Let $E, F$ be non-empty compact subsets of $\mathbb{R}^{n}$. Assume $\lambda>0$ be fixed. Then there holds

$$
\left|C_{\tau}^{l}\left(C_{\lambda}^{u}\left(\chi_{E}\right)\right)(x)-C_{\tau}^{l}\left(C_{\lambda}^{u}\left(\chi_{F}\right)\right)(x)\right| \leq 2 \sqrt{\lambda} \operatorname{dist}_{\mathcal{H}}(E, F) \quad\left(\text { for } x \in \mathbb{R}^{n}, \tau>0\right) .
$$


The Stable Ridge Transform for characteristic functions is defined next.

Definition 5.8. Let $E$ be a non-empty compact subset of $\mathbb{R}^{n}$. We define the stable ridge transform of $E$ by

$$
S R_{\lambda, \tau}\left(\chi_{E}\right)(x)=R_{\tau}\left(C_{\lambda}^{u}\left(\chi_{E}\right)\right)(x) \quad x \in \mathbb{R}^{n}, \quad \lambda>0, \quad \tau>0,
$$

The stable ridge transform $S R_{\lambda, \tau}\left(\chi_{E}\right)(x)$ is Hausdorff-Lipschitz continuous. This is the content of the following Theorem.

Theorem 5.9. Let $E, F$ be non-empty compact subsets of $\mathbb{R}^{n}$. Assume $\lambda>0$ and $\tau>0$. Then, there holds

$$
\left|S R_{\lambda, \tau}\left(\chi_{E}\right)(x)-S R_{\lambda, \tau}\left(\chi_{F}\right)(x)\right| \leq 4 \sqrt{\lambda} \operatorname{dist}_{\mathcal{H}}(E, F) \quad\left(\text { for } x \in \mathbb{R}^{n}\right) .
$$

Figure 5 illustrates the content of Theorem 5.9. Figure 5(a) displays a domain $E$ represented by a binary image of a cat, $(c)$ shows a domain $F$ obtained by randomly sampling $E$, whereas $(b)$ and $(d)$ picture a suplevel set of the stable ridge transforms of the respective characteristic functions.

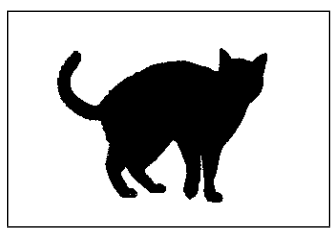

(a)

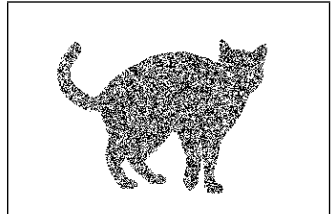

(c)

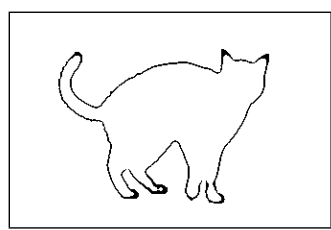

(b)

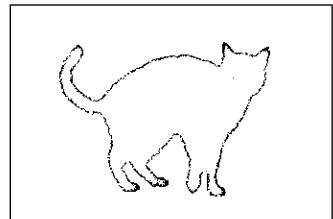

$(d)$

Fig. 5. (a) Domain $E$ given by the image of a cat displayed here as $1-\chi_{E} ;(b)$ Boundary extraction using the stable ridge transform, $S R_{\lambda, \tau}\left(\chi_{E}\right)$ ), for $\lambda=0.1$ and $\tau=\lambda / 8$; (c) Domain $F$ obtained by randomly sampling $E ;(d)$ Boundary extraction of the data sample after thresholding the stable ridge transform, $S R_{\lambda, \tau}\left(\chi_{F}\right)$ ), computed for $\lambda=0.1$ and $\tau=\lambda / 8$.

Remark 5.10. Similarly to the Stable Ridge Transform of a non-empty compact subset $E$ of $\mathbb{R}^{n}$, we can then define the Stable Valley Transform of $E$ for $\lambda>\tau$ as

$$
S V_{\lambda, \tau}\left(\chi_{E}\right)(x)=V_{\tau}\left(C_{\lambda}^{u}\left(\chi_{E}\right)\right)(x) \quad x \in \mathbb{R}^{n}, \quad \lambda>\tau>0,
$$

and the Stable Edge Transform of $E$ for $\lambda>\tau$ as

$$
S E_{\lambda, \tau}\left(\chi_{E}\right)(x)=E_{\tau}\left(C_{\lambda}^{u}\left(\chi_{E}\right)\right)(x) \quad x \in \mathbb{R}^{n}, \quad \lambda>\tau>0 .
$$


The condition $\lambda>\tau$ is invoked because from Theorem 2.1(iii) in Ref. 48, we have that

$$
\left.C_{\tau}^{u}\left(C_{\lambda}^{u}(f)\right)\right)=\left\{\begin{array}{l}
C_{\lambda}^{u}(f), \text { for } \lambda \leq \tau \\
C_{\tau}^{u}(f), \text { for } \lambda \geq \tau
\end{array}\right.
$$

Hence, if $\lambda \leq \tau$, we would get $S V_{\lambda, \tau}\left(\chi_{E}\right)(x)=0$ and $S E_{\lambda, \tau}\left(\chi_{E}\right)(x)$ would simply equal to $S R_{\lambda, \tau}\left(\chi_{E}\right)(x)$. The Hausdorff stability of $S V_{\lambda, \tau}\left(\chi_{E}\right)(x)$ follows from Theorem 5.5 and the triangle inequality.

\section{Prototype examples}

To justify the stable ridge transform as a tool to extract exterior corners and lower dimensional objects, we present some prototype examples where it is possible to derive an explicit expression for the stable ridge transform. To simplify the notation, for $\lambda>0$ and $\tau>0$ we introduce the following positive constants

$$
\alpha=\frac{1}{\sqrt{\lambda}}-\sqrt{\frac{\tau}{\lambda(\lambda+\tau)}} \quad \text { and } \quad \beta=\sqrt{\frac{\lambda+\tau}{\lambda \tau}}-\frac{1}{\sqrt{\lambda}} .
$$

Example 6.1. We consider first some one-dimensional examples to show the different effects of $\left.S R_{\lambda, \tau}\left(\chi_{K}\right)\right)$ when $K$ is $(i)$ a single point set $K_{0}=\{0\}$, and (ii) a single jump $K_{-}=(-\infty, 0]$.

Case (i): For this case, with the constants $\alpha$ and $\beta$ defined by (6.1), we have for $x \in \mathbb{R}$

$$
\begin{aligned}
& C_{\lambda}^{u}\left(\chi_{K_{0}}\right)(x)= \begin{cases}\lambda(|x|-1 / \sqrt{\lambda})^{2} & \text { if }|x| \leq 1 / \sqrt{\lambda}, \\
0 & \text { if }|x| \geq 1 / \sqrt{\lambda} ;\end{cases} \\
& C_{\tau}^{l}\left(C_{\lambda}^{u}\left(\chi_{K_{0}}\right)\right)(x)= \begin{cases}\frac{\sqrt{\lambda}}{\lambda+\tau}-\tau x^{2} & \text { if }|x| \leq \frac{\sqrt{\lambda}}{\lambda+\tau}, \\
C_{\lambda}^{u}\left(\chi_{K_{0}}\right)(x) & \text { if }|x| \geq \frac{\sqrt{\lambda}}{\lambda+\tau} ;\end{cases} \\
& S R_{\lambda, \tau}\left(\chi_{K_{0}}\right)(x)= \begin{cases}(\lambda+\tau)\left(|x|-\frac{\sqrt{\lambda}}{\lambda+\tau}\right)^{2} \text { if }|x| \leq \frac{\sqrt{\lambda}}{\lambda+\tau}, \\
0 & \text { if }|x| \geq \frac{\sqrt{\lambda}}{\lambda+\tau} .\end{cases}
\end{aligned}
$$

The function $S R_{\lambda, \tau}\left(\chi_{K_{0}}\right)(x)$ attains its maximum at $x=0$ with $S R_{\lambda, \tau}\left(\chi_{K_{0}}\right)(0)=\lambda /(\lambda+\tau)$. 
Case (ii): For this case, with the constants $\alpha$ and $\beta$ defined by (6.1), we have for $x \in \mathbb{R}$

$$
\begin{aligned}
& C_{\lambda}^{u}\left(\chi_{K_{-}}\right)(x)= \begin{cases}0, & \text { if } x \geq 1 / \sqrt{\lambda}, \\
\lambda(x-1 / \sqrt{\lambda})^{2}, & \text { if } 1 / \sqrt{\lambda} \geq x \geq 0, \\
1, & \text { if } x \leq 0 ;\end{cases} \\
& C_{\tau}^{l}\left(C_{\lambda}^{u}\left(\chi_{K_{-}}\right)\right)(x)= \begin{cases}1-\tau(x+\beta)^{2}, & \text { if }-\beta \leq x \leq \alpha, \\
C_{\lambda}^{u}\left(\chi_{K_{-}}\right)(x), & \text { otherwise; }\end{cases} \\
& S R_{\lambda, \tau}\left(\chi_{K_{-}}\right)(x)= \begin{cases}(\lambda+\tau)(x-\alpha)^{2}, & \text { if } 0 \leq x \leq \alpha, \\
\tau(x+\beta)^{2}, & \text { if }-\beta \leq x \leq 0, \\
0, & \text { otherwise. }\end{cases}
\end{aligned}
$$

The function $S R_{\lambda, \tau}\left(\chi_{K_{-}}\right)(x)$ attains its maximum at $x=0$ with $S R_{\lambda, \tau}\left(\chi_{K_{-}}\right)(0)=(\sqrt{\lambda+\tau}-\sqrt{\tau})^{2} / \lambda$. For $\lambda>0, \tau>0$ we have moreover that for $x \in \mathbb{R}$

$$
\begin{aligned}
S R_{\lambda, \tau}\left(\chi_{K_{-}}\right)(0)<S R_{\lambda, \tau}\left(\chi_{K_{0}}\right)(0) & \Longleftrightarrow(\sqrt{\lambda+\tau}-\sqrt{\tau})^{2} / \lambda<\lambda /(\lambda+\tau) \\
& \Longleftrightarrow 1 /(\sqrt{\lambda+\tau}+\sqrt{\tau})^{2}<1 /(\lambda+\tau),
\end{aligned}
$$

which is clearly true since $\lambda, \tau>0$.

Figure 6 displays the graph of the transforms $\left.S R_{\lambda, \tau}\left(\chi_{K}\right)\right)$ in the case of $K=K_{0}$ and $K=K_{-}$.

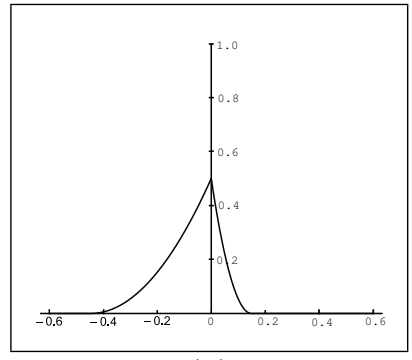

$(a)$

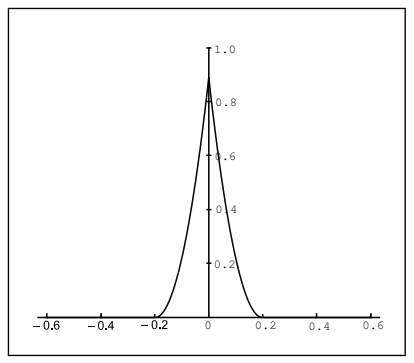

(b)

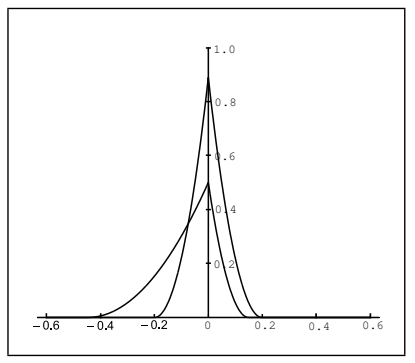

(c)

Fig. 6. (a) Graph of $S R_{\lambda, \tau}\left(\chi_{K_{-}}\right)$; (b) Graph of $S R_{\lambda, \tau}\left(\chi_{K_{0}}\right)$; (c) Graphs of both the transforms for $\lambda=20$ and $\tau=\lambda / 8$.

We will present the explicit calculations needed to determine $C_{\tau}^{l}\left(C_{\lambda}^{u}\left(\chi_{K_{-}}\right)\right)$and $S R_{\lambda, \tau}\left(\chi_{K_{-}}\right)$, because these will be used in the proof of our 'Height Theorem' (Theorem 6.4) below for regular points on the boundary of a domain in $\mathbb{R}^{n}$.

The expression of the upper transform $C_{\lambda}^{u}\left(\chi_{K_{-}}\right)$can be obtained quite easily, hence we focus only on deriving an expression for the lower transform of 
$C_{\lambda}^{u}\left(\chi_{K_{-}}\right)$. For this purpose, by definition, we need to compute the convex envelope of $C_{\lambda}^{u}\left(\chi_{K_{-}}\right)+\tau|\cdot|^{2}$. Given

$$
C_{\lambda}^{u}\left(\chi_{K_{-}}\right)(x)+\tau|x|^{2}= \begin{cases}\tau x^{2} & \text { if } x \geq 1 / \sqrt{\lambda}, \\ \lambda(x-1 / \sqrt{\lambda})^{2}+\tau x^{2} & \text { if } 1 / \sqrt{\lambda} \geq x \geq 0, \\ 1+\tau x^{2} & \text { if } x \leq 0\end{cases}
$$

we observe that (6.5) is a piecewise quadratic function with each piece a convex function. In order to find its convex envelope, we look for the common tangent line of the following two functions $f_{0}$ and $f_{1}$, representing restrictions of (6.5), and show that this is a supporting line of the whole function. Given

$$
\begin{aligned}
& f_{0}(x)=1+\tau x^{2} \quad \text { for } x \leq 0 \\
& \text { and } f_{1}(x)=\lambda(x-1 / \sqrt{\lambda})^{2}+\tau x^{2} \text { for } 0 \leq x \leq 1 / \sqrt{\lambda} \text {, }
\end{aligned}
$$

the tangent line to $f_{0}$ is $\ell_{0}(x)=1-\tau x_{0}^{2}+2 \tau x_{0} x$ with $x_{0} \leq 0$ and the tangent line to $f_{1}$ is $\ell_{1}(x)=1-(\lambda+\tau) x_{1}^{2}-2 \sqrt{\lambda} x+2(\lambda+\tau) x_{1} x$ with $0 \leq x_{1} \leq 1 / \sqrt{\lambda}$. As the common tangent line must satisfy $\ell_{0} \equiv \ell_{1}$, by comparing the coefficients, $\left(x_{0}, x_{1}\right)$ must then solve the system

$$
\left\{\begin{array}{l}
1-\tau x_{0}^{2}=1-(\lambda+\tau) x_{1}^{2} \\
2 \tau x_{0}=-2 \sqrt{\lambda}+2(\lambda+\tau) x_{1}
\end{array}\right.
$$

whose solution yields

$$
x_{0}=\frac{1}{\sqrt{\lambda}}-\sqrt{\frac{\lambda+\tau}{\lambda \tau}}=-\beta, \quad x_{1}=\frac{1}{\sqrt{\lambda}}-\sqrt{\frac{\tau}{\lambda(\lambda+\tau)}}=\alpha .
$$

Since $x_{0}<0$ and $0<x_{1}<1 / \sqrt{\lambda}$, the common tangent line to the pieces $f_{0}$ and $f_{1}$ is then given by

$$
\ell_{0}(x)=1-\tau x_{0}^{2}+2 \tau x_{0} x
$$

Clearly $\ell_{0}(x) \leq f_{0}(x)$ for $x \leq 0$ and $\ell_{0}(x) \leq f_{1}(x)$ for $1 \leq x \leq 1 / \sqrt{\lambda}$. Furthermore, we have $\ell_{0}\left(x_{0}\right)=f_{0}\left(x_{0}\right)$ and $\ell_{0}\left(x_{1}\right)=f_{1}\left(x_{1}\right)$. To complete the proof, we only need to show that $\ell_{0}(x)<\tau x^{2}$ for $x>1 / \sqrt{\lambda}$. For $\lambda>0, \tau>0$ and $x>1 / \sqrt{\lambda}$ we have

$$
\begin{aligned}
\ell_{0}\left(x_{0}\right)<\tau x^{2} & \Longleftrightarrow 1-\tau x_{0}^{2}+2 \tau x_{0} x<\tau x^{2} \\
& \Longleftrightarrow \tau\left(x-x_{0}\right)^{2}>1 \\
& \Longleftrightarrow x-x_{0}>1 / \sqrt{\tau} \\
& \Longleftrightarrow x_{0} \leq 1 / \sqrt{\lambda}-1 / \sqrt{\tau} \\
& \Longleftrightarrow 1 / \sqrt{\tau} \leq \sqrt{\frac{\lambda+\tau}{\lambda \tau}} .
\end{aligned}
$$


which is clearly true since $\lambda>0$ and $\tau>0$. Here we have used the definition of $x_{0}$ in (6.7) and the fact that $x>1 / \sqrt{\lambda}$. It thus follows that, for $x \in \mathbb{R}$,

$$
\operatorname{co}\left[C_{\lambda}^{u}\left(\chi_{K_{-}}\right)+\tau|\cdot|^{2}\right](x)= \begin{cases}1+\tau x^{2}, & \text { if } x \leq x_{0}, \\ \ell_{0}(x), & \text { if } x_{0} \leq x \leq x_{1}, \\ \lambda(x-1 / \sqrt{\lambda})^{2}+\tau x^{2}, & \text { if } x_{1} \leq x \leq 1 / \sqrt{\lambda}, \\ \tau x^{2}, & \text { if } x \geq 1 / \sqrt{\lambda} .\end{cases}
$$

By subtracting (6.10) to $\tau|x|^{2}$ we obtain first $C_{\tau}^{l}\left(C_{\lambda}^{u}\left(\chi_{K_{-}}\right)\right)$as given in (6.3), and then by substracting $C_{\lambda}^{u}\left(\chi_{K_{-}}\right)$to $C_{\tau}^{l}\left(C_{\lambda}^{u}\left(\chi_{K_{-}}\right)\right)$we can verify the explicit expression for $S R_{\lambda, \tau}\left(\chi_{K_{-}}\right)$given in (6.3).

Next we show that given an open subset $\Omega$ of $\mathbb{R}^{n}$, along the normal direction to a $\delta$-regular point $x_{0}$ of $\partial \Omega, S R_{\lambda, \tau}\left(\chi_{\bar{\Omega}}\right)$ reaches its maximum at $x_{0}$, and the height of the stable ridge transform at $x_{0}$ is given then by $S R_{\lambda, \tau}\left(\chi_{\bar{\Omega}}\right)\left(x_{0}\right)=(\sqrt{\lambda+\tau}-$ $\sqrt{\tau})^{2} / \lambda$. To establish this result, we first need the following lemma.

Lemma 6.2. Assume that $\lambda>0, \tau>0$, the positive constants $\alpha$ and $\beta$ are as defined by (6.1), and $r>\max \{1 / \sqrt{\lambda}, \beta\}$. Let $B(0 ; r) \subset \mathbb{R}^{n}$ be the open ball centred at 0 with radius $r$. Then, for $x \in \mathbb{R}^{n}$,

$$
\begin{aligned}
& C_{\lambda}^{u}\left(\chi_{\bar{B}(0 ; r)}\right)(x)= \begin{cases}1, & \text { if }|x| \leq r, \\
\lambda(|x|-r-1 / \sqrt{\lambda})^{2}, & \text { if } r \leq|x| \leq r+1 / \sqrt{\lambda}, \\
0, & \text { if } r+1 / \sqrt{\lambda} \leq|x| ;\end{cases} \\
& C_{\tau}^{l}\left(C_{\lambda}^{u}\left(\chi_{\bar{B}(0 ; r)}\right)(x)= \begin{cases}1-\tau(|x|-r+\beta)^{2}, & \text { if } r-\beta \leq|x| \leq r+\alpha, \\
C_{\lambda}^{u}\left(\chi_{\bar{B}(0 ; r)}\right)(x), & \text { otherwise; }\end{cases} \right. \\
& \operatorname{SR}_{\lambda, \tau}\left(\chi_{\bar{B}(0 ; r)}\right)(x)= \begin{cases}\tau(|x|-r+\beta)^{2}, & \text { if } r-\beta \leq|x| \leq r, \\
(\lambda+\tau)(|x|-r-\alpha)^{2}, & \text { if } r \leq|x| \leq r+\alpha, \\
0, & \text { otherwise. }\end{cases}
\end{aligned}
$$


For the complement $B^{c}(0 ; r)$ of $B(0 ; r)$, we have, for $x \in \mathbb{R}^{n}$,

$$
\begin{aligned}
& C_{\lambda}^{u}\left(\chi_{B^{c}(0 ; r)}\right)(x)= \begin{cases}0, & \text { if }|x| \leq r-1 / \sqrt{\lambda}, \\
\lambda(|x|-r+1 / \sqrt{\lambda})^{2}, & \text { if } r-1 / \sqrt{\lambda} \leq|x| \leq r, \\
1, & \text { if } r \leq|x|\end{cases} \\
& C_{\tau}^{l}\left(C_{\lambda}^{u}\left(\chi_{B_{r}^{c}(0)}\right)\right)(x)= \begin{cases}1-\tau(|x|-r-\beta)^{2}, & \text { if } r-\alpha \leq|x| \leq r+\beta, \\
C_{\lambda}^{u}\left(\chi_{\bar{B}^{c}(0 ; r)}\right)(x), & \text { otherwise } ;\end{cases} \\
& S R_{\lambda, \tau}\left(\chi_{B^{c}(0 ; r)}\right)(x)= \begin{cases}(\lambda+\tau)(|x|-r+\alpha)^{2}, & \text { if } r-\alpha \leq|x| \leq r, \\
\tau(|x|-r-\beta)^{2}, & \text { if } r \leq|x| \leq r+\beta, \\
0, & \text { otherwise. }\end{cases}
\end{aligned}
$$

Remark 6.3. The assumption $r>\max \{1 / \sqrt{\lambda}, \beta\}$ has been made only to ensure that $r-\beta>0$ and $r-1 / \sqrt{\lambda}>0$, for otherwise the expression of the transforms can be easily obtained, by direct inspection, specializing the ones given in (6.11). Recalll also that, in general, for $\lambda \rightarrow 0, C_{\lambda}^{l}(f)$ and $C_{\lambda}^{u}(f)$ will converge to the convex and concave envelope of $f$, respectively. So in the present example, they would be the constant functions equal to zero and to one, respectively.

Theorem 6.4. (The Height Theorem) Let $\lambda>0, \tau>0$, and $\beta$ be as defined in (6.1). Suppose that $\Omega$ is a non-empty open subset of $\mathbb{R}^{n}$ with $\bar{\Omega} \neq \mathbb{R}^{n}$. If $r>$ $\max \{1 / \sqrt{\lambda}, \beta\}$ and $x_{0} \in \partial \Omega$ is an r-regular point (that is, $\Omega$ satisfies both the $r$ interior and $r$-exterior ball condition), then $\partial \Omega$ has a unique normal line at $x_{0}$, and along the outward normal direction $\nu$ (with $|\nu|=1$ ), we have

$$
\begin{aligned}
& C_{\lambda}^{u}\left(\chi_{\bar{\Omega}}\right)\left(x_{0}+t \nu\right)=C_{\lambda}^{u}\left(\chi_{K_{-}}\right)(t), \\
& C_{\tau}^{l}\left(C_{\lambda}^{u}\left(\chi_{\bar{\Omega}}\right)\right)\left(x_{0}+t \nu\right)=C_{\tau}^{l}\left(C_{\lambda}^{u}\left(\chi_{K_{-}}\right)\right)(t), \\
& S R_{\lambda, \tau}\left(\chi_{\bar{\Omega}}\right)\left(x_{0}+t \nu\right)=S R_{\lambda, \tau}\left(\chi_{K_{-}}\right)(t)
\end{aligned}
$$

for $t \in[-r, r]$ whenever $r>1 / \sqrt{\lambda}$. The function $t \mapsto S R_{\lambda, \tau}\left(\chi_{\bar{\Omega}}\right)\left(x_{0}+t \nu\right)$, for $|t| \leq r$, reaches its maximum at $t=0$ with

$$
S R_{\lambda, \tau}\left(\chi_{\bar{\Omega}}\right)\left(x_{0}\right)=\frac{(\sqrt{\lambda+\tau}-\sqrt{\tau})^{2}}{\lambda} .
$$

We recall from Ref. 19, for instance, that we say that the boundary $\partial \Omega$ is $C^{1,1}$ at $x_{0}$ if $(i)$ there is a neighbourhood $B\left(x_{0} ; \delta\right)$ of $x_{0}$ and an orthogonal coordinate system $\left(x_{1}^{\prime}, \ldots, x_{n}^{\prime}\right)$ near $x_{0}$ such that $\partial \Omega$ is locally the graph of a function $g$; $(i i)$ the function $g$ is locally differentiable in a neighbourhood of 0 and (iii) there is a constant $L>0$ such that $\left|D g\left(x_{\hat{n}}^{\prime}\right)-D g(0)\right| \leq L\left|x_{\hat{n}}^{\prime}\right|$ in a neighbourhood of 0 , where we have set $x_{\hat{n}}^{\prime}=\left(x_{1}^{\prime}, \ldots, x_{n-1}^{\prime}\right)$. The relation between this notion of regularity and that given by Definition 2.16 is contained in the following result. 
Proposition 6.5. Let $\Omega \subset \mathbb{R}^{n}$ be a non-empty open set with $\bar{\Omega} \neq \mathbb{R}^{n}$. Then every $C^{1,1}$ point $x_{0} \in \partial \Omega$ is an $r$-regular point of $\Omega$ for $r>0$ sufficiently small, and

$$
S R_{\lambda, \tau}\left(\chi_{\bar{\Omega}}\right)\left(x_{0}\right) \leq \frac{(\sqrt{\lambda+\tau}-\sqrt{\tau})^{2}}{\lambda}
$$

The stable ridge transform of the characteristic function of an open set $\Omega \subset \mathbb{R}^{n}$ is supported in the $1 / \sqrt{\lambda}$-closed neighbourhood of the boundary $\partial \Omega$.

Proposition 6.6. Let $\lambda>0$ and $\tau>0$. Assume $\Omega$ be a non-empty open subset of $\mathbb{R}^{n}$ with $\bar{\Omega} \neq \mathbb{R}^{n}$. If $x_{0} \in \mathbb{R}^{n}$ is such that $\operatorname{dist}\left(x_{0} ; \partial \Omega\right)>1 / \sqrt{\lambda}$, then $S R_{\lambda, \tau}\left(\chi_{\bar{\Omega}}\right)\left(x_{0}\right)=0$.

Remark 6.7. By Theorem 6.4 and Proposition 6.6, if we take the suplevel sets of the stable ridge transform $S R_{\lambda, \tau}\left(\chi_{\bar{\Omega}}\right)$, that is, if we consider the set $S_{t}:=\{x \in$ $\left.\mathbb{R}^{n}, S R_{\lambda, \tau}\left(\chi_{\bar{\Omega}}\right)(x)>t\right\}$ with $0<t<(\sqrt{\lambda+\tau}-\sqrt{\tau})^{2} / \lambda$, we are able to extract at least a small neighbourhood of the set of the $1 / \sqrt{\lambda}$-regular points. Using a prototype example we will show that if we lift the threshold further (that is, if we consider a value of $t$ greater than the maximum value $\left.(\sqrt{\lambda+\tau}-\sqrt{\tau})^{2} / \lambda\right)$, the stable ridge transform can actually extract exterior corner points. We can also standardise the height of regular points by taking, for example, $\tau=\lambda / 8$. For this choice, the height at regular points will be $1 / 2$. Therefore, for $\tau=\lambda / 8$, if the stable ridge transform at a point is greater than $1 / 2$, we say that the point is $\lambda$-singular.

The preceding observation motivates the following definition of extractable corner points.

Definition 6.8. Assume $\Omega$ be a bounded open subset of $\mathbb{R}^{n}$. A point $x_{0} \in \partial \Omega$ is called an extractable corner point of $\Omega$ if for at least sufficiently large $\lambda>0$ and $\tau>0$,

$$
S R_{\lambda, \tau}\left(\chi_{\Omega}\right)\left(x_{0}\right)>\mu_{1}(\lambda, \tau)
$$

where

$$
\mu_{1}(\lambda, \tau):=\frac{(\sqrt{\lambda+\tau}-\sqrt{\tau})^{2}}{\lambda},
$$

is called the standard height for codimension-1 regular boundary points.

To show an application of Definition 6.8, we give below the expression of $S R_{\lambda, \tau}\left(\chi_{K}\right)$ for the case of $K:=K_{0}^{(n)}=\{0\} \subset \mathbb{R}^{n}$. This example is a straightforward generalisation to $\mathbb{R}^{n}$, by a simple rotation, of the Case $(i)$ of Example 6.1 . 
Example 6.9. Let $K_{0}^{(n)}=\{0\} \subset \mathbb{R}^{n}$, then, for $x \in \mathbb{R}^{n}$,

$$
\begin{aligned}
& C_{\lambda}^{u}\left(\chi_{K_{0}^{(n)}}\right)(x)= \begin{cases}\lambda(|x|-1 / \sqrt{\lambda})^{2} & \text { if }|x| \leq 1 / \sqrt{\lambda}, \\
0 & \text { if }|x| \geq 1 / \sqrt{\lambda} ;\end{cases} \\
& C_{\tau}^{l}\left(C_{\lambda}^{u}\left(\chi_{K_{0}^{(n)}}\right)\right)(x)= \begin{cases}\frac{\tau}{\lambda+\tau}-\tau x^{2} & \text { if }|x| \leq \frac{\sqrt{\lambda}}{\lambda+\tau}, \\
C_{\lambda}^{u}\left(\chi_{K_{0}^{(n)}}\right)(x) & \text { if }|x| \geq \frac{\sqrt{\lambda}}{\lambda+\tau} ;\end{cases} \\
& S R_{\lambda, \tau}\left(\chi_{K_{0}^{(n)}}\right)(x)= \begin{cases}(\lambda+\tau)\left(|x|-\frac{\sqrt{\lambda}}{\lambda+\tau}\right)^{2} & \text { if }|x| \leq \frac{\sqrt{\lambda}}{\lambda+\tau}, \\
0 & \text { if }|x| \geq \frac{\sqrt{\lambda}}{\lambda+\tau} ;\end{cases}
\end{aligned}
$$

The transform $S R_{\lambda, \tau}\left(\chi_{K_{0}^{(n)}}\right)$ attains its maximum value at $x=0$ with

$$
S R_{\lambda, \tau}\left(\chi_{K_{0}^{(n)}}\right)(0)=\frac{\lambda}{\lambda+\tau}>\frac{(\sqrt{\lambda+\tau}-\sqrt{\tau})^{2}}{\lambda} .
$$

As a result of the inequality (6.19), it follows that if a regular lower dimensional manifold is present in the data set, for example, if we consider $K \cup \bar{\Omega} \subset \mathbb{R}^{n}$, where $K$ is a smooth compact manifold of dimension $\leq n-1$, then for large $\lambda$ and $\tau$, the values of $S R_{\lambda, \tau}\left(\chi_{K \cup \bar{\Omega}}\right)$ on $K$ are extractable singularities according to Definition 6.8. This effect is visualized in the numerical experiments displayed in Figure 7, Figure 8 and Figure 9.

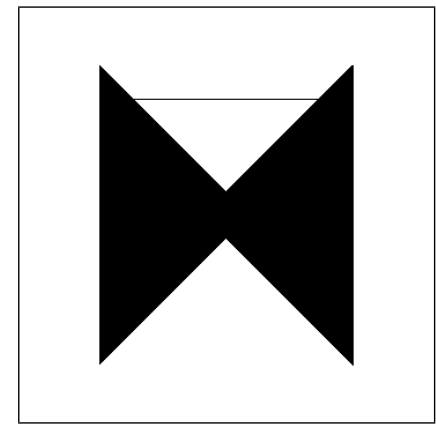

$(a)$

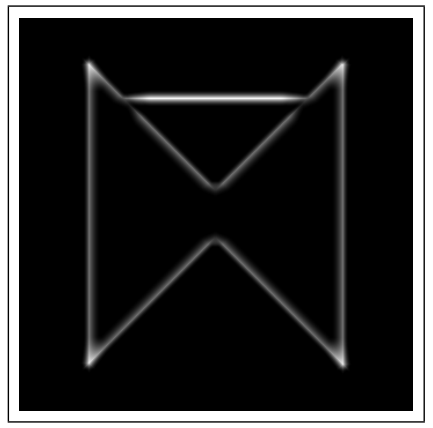

$(b)$

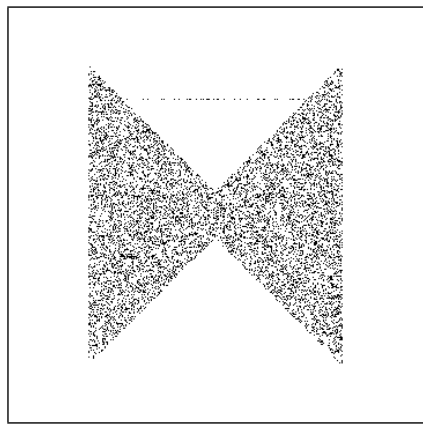

$(c)$

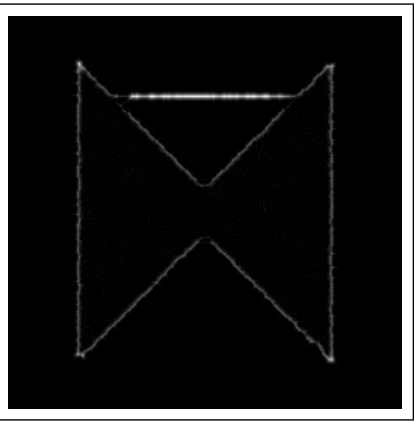

$(d)$

Fig. 7. Multiscale extraction of exterior edges and lower dimensional objects by the stable $2 d$ corner transform. (a) Original shape; (b) A suplevel set of the stable ridge transform of the object shown in $(a) ;(c)$ Randomly sample of the object shown in $(a) ;(d)$ A suplevel set of the stable ridge transform of the object shown in $(c)$.

Notice that Theorem 6.4 tells us nothing about what happens at the singular points. It might happen that at some $r$-singular points of $\partial \Omega, R_{\tau}\left(C_{\lambda}^{u}\left(\chi_{\bar{\Omega}}\right)\right)$ takes on values lower than at the regular points of $\partial \Omega$. This indeed happens, for instance, at the interior corners (see Proposition 6.10 below). By contrast, by means of a prototype example of an exterior corner, we will see that, using Definition 6.8, 


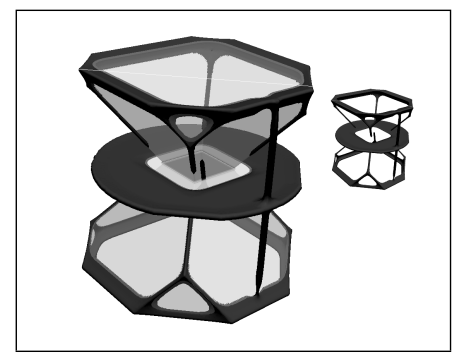

$(a)$

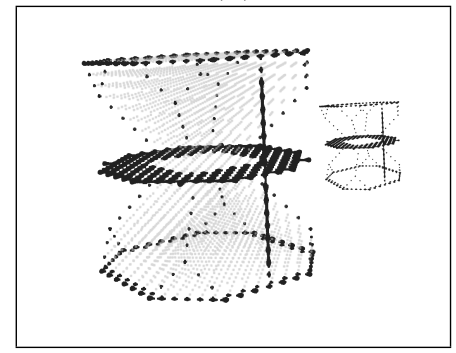

$(c)$

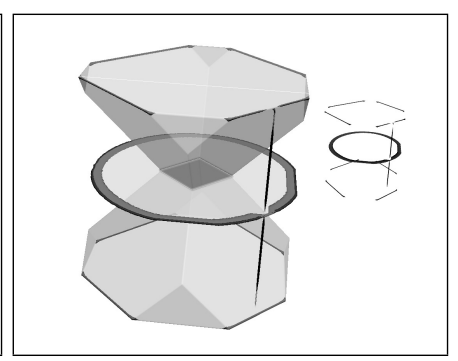

(b)

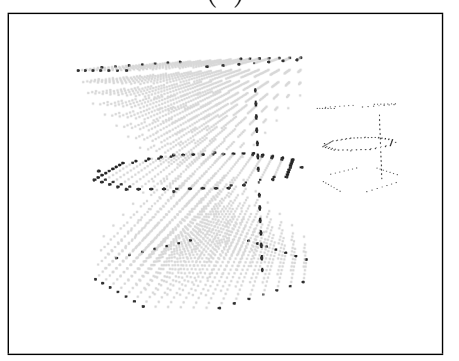

$(d)$

Fig. 8. Multiscale extraction of exterior edges and lower dimensional objects by the stable $3 d$ corner transform for the case of well sampled object with different displayed suplevel sets in $(a)$ and $(b)$; and for the same object loosely sampled with different displayed suplevel sets in $(c)$ and $(d)$. For sake of visualization, the extracted exterior edges and lower dimensional objects are also displayed separately, as well as superimposed to the original object.

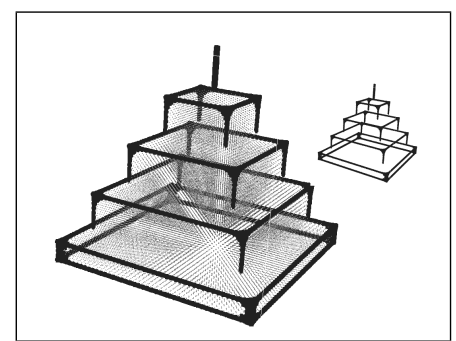

$(a)$

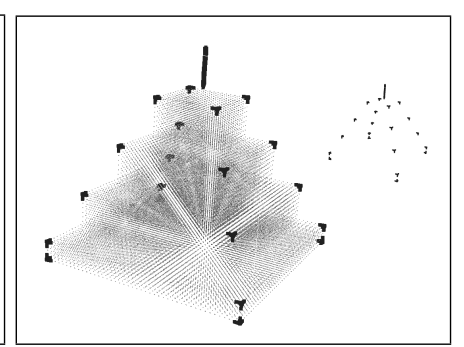

(b)

Fig. 9. Stable ridge transform for a sampled nonconvex polytope: $(a)$ Exterior edges; $(b)$ Exterior vertices

$R_{\tau}\left(C_{\lambda}^{u}\left(\chi_{\bar{\Omega}}\right)\right)$ can detect exterior corners. However, even in this case, it can happen that on isolated lower dimensional objects, such as curves or isolated points, $R_{\tau}\left(C_{\lambda}^{u}\left(\chi_{\bar{\Omega}}\right)\right)$ can take on values higher than those at the near external corner point. It follows that, for these cases, a different Hausdorff stable method will be therefore needed to detect interior corners and boundary intersections of domains.

Proposition 6.10. Assume $\Omega$ to be a bounded and open subset of $\mathbb{R}^{n}$. Let $x_{0} \in$ 
$\partial \Omega$ be an interior $r$-regular point, that is, there is a ball $B\left(x^{\prime} ; r\right) \subset \Omega$ such that $x_{0} \in \partial B\left(x^{\prime} ; r\right)$. Then $x_{0}$ is not extractable in the sense of Definition 6.8 , that is, for $1 / \sqrt{\lambda} \leq r$,

$$
0 \leq S R_{\lambda, \tau}(f)\left(x_{0}\right) \leq \mu_{1}(\lambda, \tau)
$$

Using the example of an explicit prototype exterior corner in $\mathbb{R}^{2}$, we next show that $R_{\tau}\left(C_{\lambda}^{u}\left(\chi_{\bar{\Omega}}\right)\right)$ can detect exterior corners following Definition 6.8.

Example 6.11. Assume $a>0, \lambda>0$ and $\tau>0$ and denote by $\alpha$ and $\beta$ the positive constants defined by (6.1). Let $K_{a}:=\left\{(x, y) \in \mathbb{R}^{2},|y| \leq a x, x \geq 0\right\}$. The upper transform of $\chi_{K_{a}}$ has the following expression for $(x, y) \in \mathbb{R}^{2}$,

$C_{\lambda}^{u}\left(\chi_{K_{a}}\right)(x, y)= \begin{cases}1, & \text { if }|y| \leq a x \text { and } x \geq 0 \\ \lambda\left(\frac{-a x+|y|}{\sqrt{1+a^{2}}}-\frac{1}{\sqrt{\lambda}}\right)^{2}, & \text { if } 0 \leq \frac{-a x+|y|}{\sqrt{1+a^{2}}} \leq \frac{1}{\sqrt{\lambda}} \text { and } \frac{x+a|y|}{\sqrt{1+a^{2}}} \geq 0 \\ \lambda\left(\sqrt{x^{2}+y^{2}}-\frac{1}{\sqrt{\lambda}}\right)^{2}, & \text { if } \sqrt{x^{2}+y^{2}} \leq \frac{1}{\sqrt{\lambda}} \text { and } \frac{x+a|y|}{\sqrt{1+a^{2}}} \leq 0 \\ 0, & \text { if } \sqrt{x^{2}+y^{2}} \geq \frac{1}{\sqrt{\lambda}} \text { and } \frac{x+a|y|}{\sqrt{1+a^{2}}} \leq 0\end{cases}$

whereas the mixed transform $C_{\tau}^{l}\left(C_{\lambda}^{u}\left(\chi_{K_{a}}\right)\right)$ is given by, for $(x, y) \in \mathbb{R}^{2}$,

$C_{\tau}^{l}\left(C_{\lambda}^{u}\left(\chi_{K_{a}}\right)\right)(x, y)=$

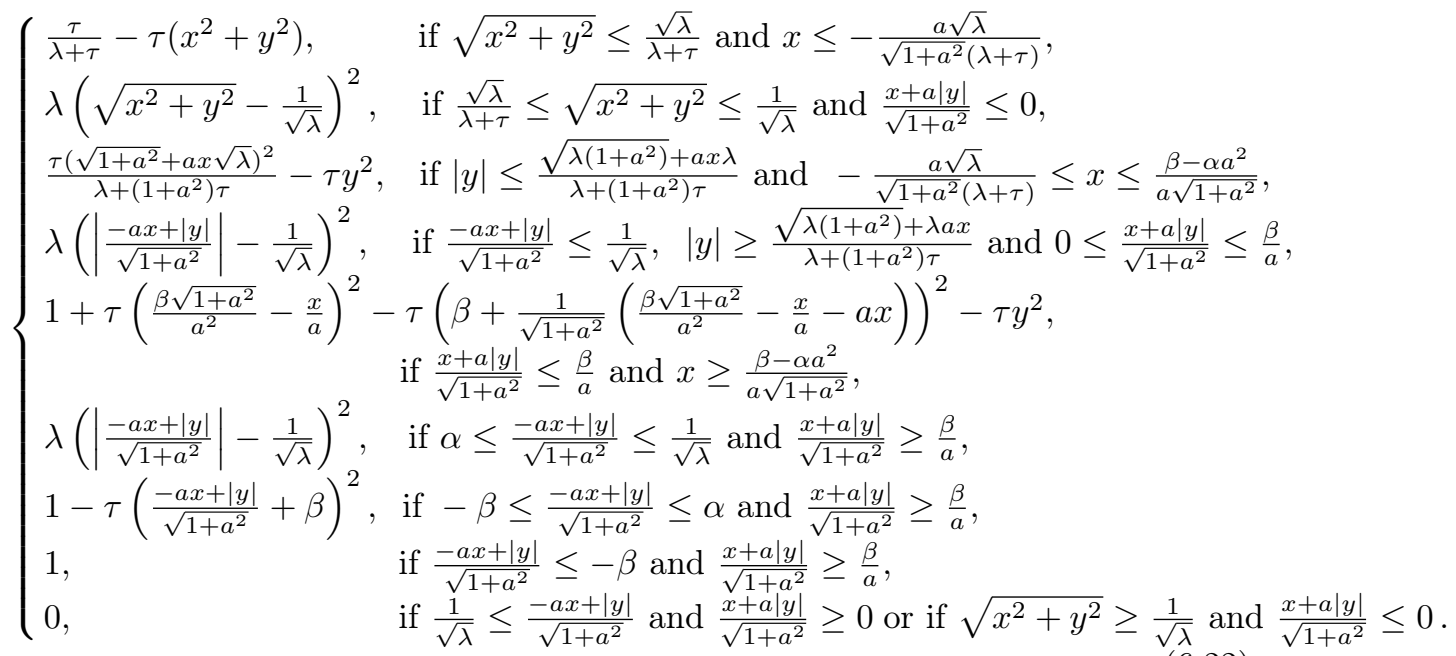


The value of $S R_{\lambda, \tau}\left(\chi_{K_{a}}\right)$ at the corner tip $(0,0)$ of $K_{a}$ is given by

$$
\mu_{2}(a, \lambda, \tau):=S R_{\lambda, \tau}\left(\chi_{K_{a}}\right)(0,0)= \begin{cases}\frac{\lambda}{\lambda+\left(1+a^{2}\right) \tau} \quad \text { if } a^{2} \leq \sqrt{\frac{\lambda+\tau}{\tau}} \\ \frac{1+a^{2} \frac{(\sqrt{\lambda+\tau}-\sqrt{\tau})^{2}}{a^{2}}}{\lambda} \text { if } a^{2} \geq \sqrt{\frac{\lambda+\tau}{\tau}}\end{cases}
$$

We call $\mu_{2}(a, \lambda, \tau)$ defined by (6.23) the standard height of codimension-2 edges of a domain with angle $\theta$ satisfying $a=\tan (\theta / 2)$. It is easy to verify that in both cases the value of $\mu_{2}(a, \lambda, \tau)$ is greater than that at a $1 / \sqrt{\lambda}$-regular point, that is,

$$
\mu_{2}(a, \lambda, \tau)>\mu_{1}(\lambda, \tau), \text { for } a>0, \text { and } \lim _{a \rightarrow+\infty} \mu_{2}(a, \lambda, \tau)=\mu_{1}(\lambda, \tau) .
$$

The limit above simply says that when the angle approaches $\pi$, the singularity at $(0,0)$ will disappear. If we further assume that $\tau$ is proportional to $\lambda$, say, $\tau=\sigma \lambda$ for some fixed $\sigma>0$, we obtain

$$
\begin{gathered}
\mu_{1}(\lambda, \sigma \lambda)=(\sqrt{1+\sigma}-\sqrt{\sigma})^{2}=\mu_{1}(1, \sigma), \\
\mu_{2}(a, \lambda, \sigma \lambda)=\mu_{2}(a, 1, \sigma)= \begin{cases}\frac{1}{1+\left(1+a^{2}\right) \sigma} & \text { if } a^{2} \leq \sqrt{\frac{1+\sigma}{\sigma}} \\
\frac{1+a^{2}}{a^{2}}(\sqrt{1+\sigma}-\sqrt{\sigma})^{2} & \text { if } a^{2} \geq \sqrt{\frac{1+\sigma}{\sigma}} .\end{cases}
\end{gathered}
$$

Figure 10 displays the graph of the upper and mixed transform of $\chi_{K_{a}}$ and of the stable ridge transform of $K_{a}$

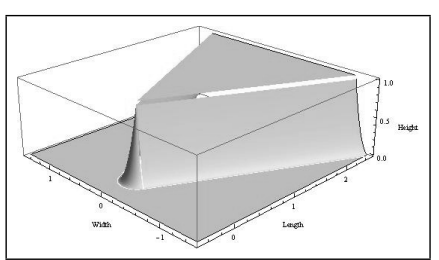

(a)

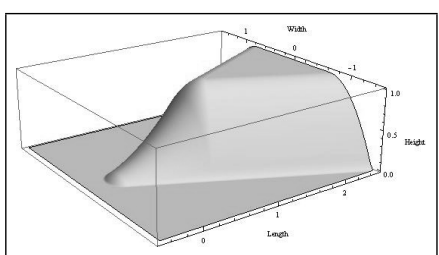

(b)

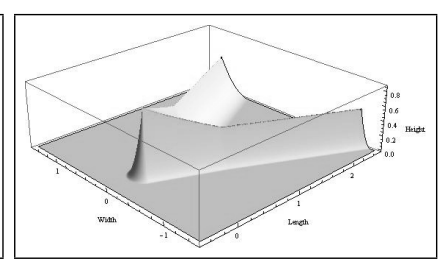

(c)

Fig. 10. Let $K_{a}:=\left\{(x, y) \in \mathbb{R}^{2},|y| \leq a x, x \geq 0\right\}$. The figure displays the graph of: $(a)$ the upper transform $C_{\lambda}^{u}\left(\chi_{K_{a}}\right) ;(b)$ the mixed transform $C_{\tau}^{l}\left(C_{\lambda}^{u}\left(\chi_{K_{a}}\right)\right)$ and $(c)$ the stable ridge transform $S R_{\lambda, \tau}\left(\chi_{K_{a}}\right)$ (for $a=0.5, \lambda=16, \tau=\lambda / 8$ ).

Remark 6.12. Since a prototype interior corner is defined as the complement of an exterior corner, one could think of detecting interior corners of $\Omega$ by looking at the stable ridge transform of the complement of $\Omega$ in $\mathbb{R}^{n}$. But this would not provide useful information for geometric objects subject to finite sampling, which is the principal type of application we have in mind. On the other hand, traditional methods, such as Harris and Susan, as well as other local mask based corner detection methods, would also not apply directly to such a situation. Thus alternative 
methods are needed to suppress lower dimensional objects. The detection of interior corners is important when we try to find intersection 'curves' of two solid bodies which are defined by samples. We will discuss these aspects in a future work.

Just for comparison purposes, assume $a>0, \lambda>0$ and $\tau>0$ and consider now the set $K_{a^{c}}:=\left(K_{a}\right)^{c}=\mathbb{R}^{2} \backslash K_{a}$, i.e. $K_{a^{c}}$ is the complement of $K_{a}$ in $\mathbb{R}^{2}$ with $K_{a}=\left\{(x, y) \in \mathbb{R}^{2}:|y| \leq a x, x \geq 0\right\}$, already introduced in Example 6.11. The set $K_{a^{c}}$ has an interior corner at the point $(0,0)$. Also for this case, it is possible to obtain closed form expressions for the upper and mixed transform of $\chi_{K_{a} c}$, and the stable ridge transform of $K_{a^{c}}$. We limit ourselves, however, to display in Figure 11 only the graphs of such transforms for two different combinations of $a, \lambda$ and $\tau$ and to note that $S R_{\lambda, \tau}\left(\chi_{K_{a} c}\right)(0,0)$ is lower than $\mu_{1}(\lambda, \tau)$.

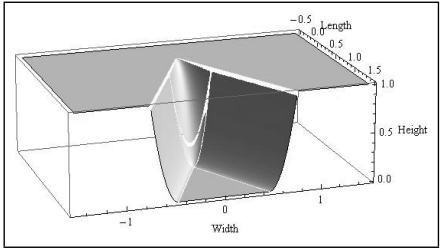

$(a)$

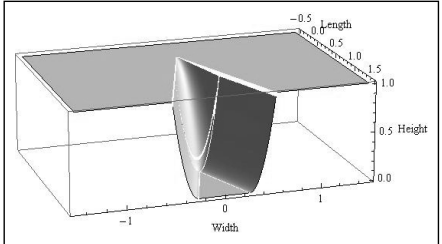

$(d)$

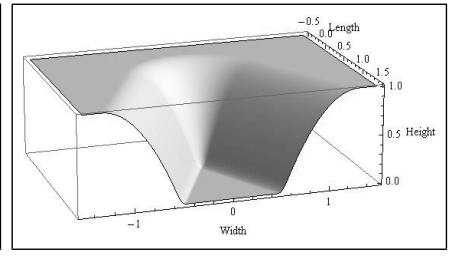

(b)

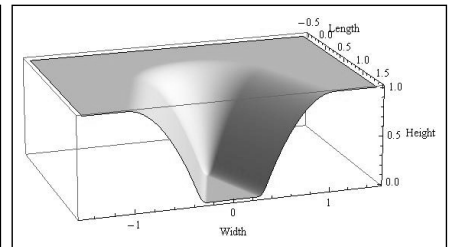

$(e)$

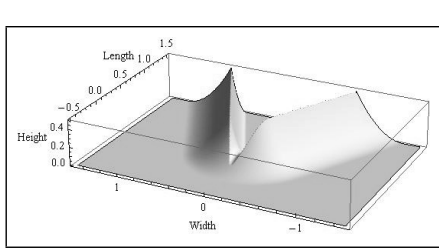

$(c)$

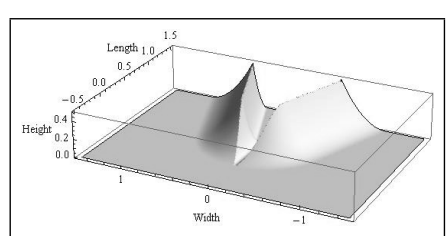

$(f)$

Fig. 11. Let $K_{a^{c}}:=\left(K_{a}\right)^{c}=\mathbb{R}^{2} \backslash K_{a}$, with $K_{a}=\left\{(x, y) \in \mathbb{R}^{2}:|y| \leq a x, x \geq 0\right\}$. Graph of: (a) the upper transform of $\chi_{K_{a} c} ;(b)$ the mixed transform of $\chi_{K_{a^{c}}}$ and $(c)$ the stable ridge transform of $K_{a^{c}}$ for $a=0.5, \lambda=16$ and $\tau=\lambda / 8\left(a^{2} \lambda-\tau>0\right)$. Graph of: $(d)$ the upper transform of $\chi_{K_{a^{c}}}$; (e) the mixed transform of $\chi_{K_{a} c}$ and $(f)$ the stable ridge transform of $K_{a^{c}}$, for $a=1 / \sqrt{8}, \lambda=16$ and $\tau=\lambda / 8\left(a^{2} \lambda-\tau=0\right)$.

From the definition of stable ridge transform and Proposition 6.6, we can capture a neighbourhood of the corner points by taking an appropriate value for the threshold. Now we consider the limit as $\lambda \rightarrow+\infty$ of the stable ridge transform of the exterior corner domain $K_{a}$ introduced in Example 6.11. We show that such limit captures singularities of different nature by its heights.

Lemma 6.13. Assume $a>0$ and $\lambda>0$, and let $K_{a}=\left\{(x, y) \in \mathbb{R}^{2},|y| \leq a x, x \geq\right.$ 
$0\}$. Let $\tau=\sigma \lambda$ with $\sigma>0$ fixed. Then, for $(x, y) \in \mathbb{R}^{2}$,

$$
\lim _{\lambda \rightarrow \infty} R_{\sigma \lambda}\left(C_{\lambda}^{u}\left(\chi_{K_{a}}\right)\right)(x, y)= \begin{cases}0, & \text { if }|y| \neq a x \\ \mu_{1}(1, \sigma), & \text { if }|y|=a x \text { and } x>0, \\ \mu_{2}(a, 1, \sigma), & \text { if }(x, y)=(0,0) .\end{cases}
$$

The pointwise limit (6.26) contains more information than only determining the regular boundary points and exterior corners. They also provide a measure of the 'strength' of the corners based on their opening angles. However, for interior corners, we note that if the opening angle is large enough for a given $\sigma>0$, then such corners are not extractable even in a weak sense, since it does not hold that $\lim _{\lambda \rightarrow+\infty} R_{\sigma \lambda}\left(C_{\lambda}^{u}\left(\chi_{K}\right)\right)(0,0)>0$.

\section{Proofs}

Proof of Theorem 2.12. Part (i): We give the proof for $1<\alpha<2$; the case $0<\alpha \leq 1$ can be shown using a similar but simpler argument. Note first that $\left|D f(x)-D f\left(x_{0}\right)\right| \leq L\left|x-x_{0}\right|^{\alpha-1}$ for $\left|x-x_{0}\right| \leq \delta$ for some $\delta>0$ and $L>0$. Then by the locality property (see Theorem 2.4 of Ref. 48) for compensated convex transforms, there exists $\Lambda>0$ such that if $\lambda>\Lambda$ and

$$
C_{\lambda}^{l}(f)\left(x_{0}\right)=\operatorname{co}\left[f+\lambda\left|(\cdot)-x_{0}\right|^{2}\right]\left(x_{0}\right)=\sum_{i=1}^{n+1} \tau_{i}\left(f\left(x_{i}\right)+\lambda\left|x_{i}-x_{0}\right|^{2}\right)
$$

for $x_{i} \in \mathbb{R}^{n}, \tau_{i} \geq 0, i=1,2, \ldots, n+1, \sum_{i=1}^{n+1} \tau_{i}=1$ and $\sum_{i=1}^{n+1} \tau_{i} x_{i}=x_{0}$, then $x_{i} \in \bar{B}\left(x_{0} ; \delta\right)$. Now consider $F(x)=f(x)-D f\left(x_{0}\right) \cdot\left(x-x_{0}\right)$. Since for any affine function $\ell \in \operatorname{Aff}\left(\mathbb{R}^{n}\right)$, we have $C_{\lambda}^{l}(f+\ell)=C_{\lambda}^{l}(f)+\ell$, it follows that, for sufficiently large $\lambda>0$, we have

$$
\begin{aligned}
F\left(x_{0}\right)-C_{\lambda}^{l}(F)\left(x_{0}\right) & =\sum_{i=1}^{n+1} \tau_{i}\left(\left(f\left(x_{0}\right)-f\left(x_{i}\right)+D f\left(x_{0}\right) \cdot\left(x_{i}-x_{0}\right)-\lambda\left|x_{i}-x_{0}\right|^{2}\right)\right. \\
& =\sum_{i=1}^{n+1} \tau_{i}\left(-\int_{0}^{1}\left(D f\left(x_{0}+t\left(x_{i}-x_{0}\right)\right)-D f\left(x_{0}\right) \cdot\left(x_{i}-x_{0}\right)\right) d t-\lambda\left|x_{i}-x_{0}\right|^{2}\right) \\
& \leq \sum_{i=1}^{n+1} \tau_{i}\left(L\left|x_{i}-x_{0}\right|^{\alpha-1}\left|x_{i}-x_{0}\right|-\lambda\left|x_{i}-x_{0}\right|^{2}\right) \\
& =\sum_{i=1}^{n+1} \tau_{i}\left(L\left|x_{i}-x_{0}\right|^{\alpha}-\lambda\left|x_{i}-x_{0}\right|^{2}\right) \leq \frac{1}{\lambda^{\frac{\alpha}{2-\alpha}}} L^{\frac{2}{2-\alpha}}\left(\frac{\alpha}{2}\right)^{\frac{\alpha}{2-\alpha}}\left(1-\frac{\alpha}{2}\right),
\end{aligned}
$$

where the last inequality follows from the fact that the mapping $s \mapsto L s^{\alpha}-\lambda s^{2}$ attains a maximum value of $\lambda^{-\alpha /(2-\alpha)} L^{2 / 2-\alpha}(\alpha / 2)^{\alpha /(2-\alpha)}(1-\alpha / 2)$ on $(0, \infty)$ at 
$s=(L \alpha / 2 \lambda)^{\frac{1}{2-\alpha}}$.

Part (ii): In this case, note first that if $f$ is differentiable at $x_{0}$, then for any $\epsilon>0$, there is a $\delta>0$ such that

$$
\frac{\left|f(x)-f\left(x_{0}\right)-D f\left(x_{0}\right) \cdot\left(x-x_{0}\right)\right|}{\left|x-x_{0}\right|} \leq \epsilon
$$

whenever $x \in \bar{B}\left(x_{0} ; \delta\right)$. Again we have, by the locality property (see Theorem 2.4 of Ref. 48) for compensated convex transforms, there exists $\Lambda>0$ such that if $\lambda>\Lambda$, then

$$
C_{\lambda}^{l}(f)\left(x_{0}\right)=\operatorname{co}\left[f+\lambda\left|(\cdot)-x_{0}\right|^{2}\right]\left(x_{0}\right)=\sum_{i=1}^{n+1} \tau_{i}\left(f\left(x_{i}\right)+\lambda\left|x_{i}-x_{0}\right|^{2}\right)
$$

for $x_{i} \in \mathbb{R}^{n}, \tau_{i} \geq 0, i=1,2, \ldots, n+1, \sum_{i=1}^{n+1} \tau_{i}=1$ and $\sum_{i=1}^{n+1} \tau_{i} x_{i}=x_{0}$, then $x_{i} \in \bar{B}\left(x_{0} ; \delta\right)$. Thus

$$
\begin{aligned}
f\left(x_{0}\right)-C_{\lambda}^{l}(f)\left(x_{0}\right) & =\sum_{i=1}^{n+1} \tau_{i}\left(f\left(x_{0}\right)-f\left(x_{i}\right)-\lambda\left|x_{i}-x_{0}\right|^{2}\right) \\
& =-\sum_{i=1}^{n+1} \tau_{i}\left(\left(f\left(x_{i}\right)-f\left(x_{0}\right)-D f\left(x_{0}\right) \cdot\left(x_{i}-x_{0}\right)\right)+\lambda\left|x_{i}-x_{0}\right|^{2}\right) \\
& \leq \sum_{i=1}^{n+1} \tau_{i}\left(\epsilon\left|x_{i}-x_{0}\right|-\lambda\left|x_{i}-x_{0}\right|^{2}\right) \leq \frac{\epsilon^{2}}{4 \lambda}
\end{aligned}
$$

Here we have used the fact that $\sum_{i=1}^{n+1} \tau_{i} D f\left(x_{0}\right) \cdot\left(x_{i}-x_{0}\right)=0$. As in $(i)$, we have also used the fact that the maximum of the function $\epsilon t-\lambda t^{2}$ for $t \geq 0$ is $\epsilon^{2} /(4 \lambda)$.

Remark 7.1. Note that when $0<\alpha \leq 1$, (i) can be proved alternatively by first showing a corresponding estimate for $M_{\lambda}(f)$, developing ideas from Chapter 3 of Ref. 12, and then using the ordering property (2.4). On the other hand, however, (ii) and the case of $1<\alpha<2$ in (i) seem easier to establish directly for the lower transform $C_{\lambda}^{l}(f)$, because $C_{\lambda}^{l}(f)$ enjoys the property that $C_{\lambda}^{l}(f+\ell)=C_{\lambda}^{l}(f)+\ell$ for any affine function $\ell \in \operatorname{Aff}\left(\mathbb{R}^{n}\right)$ whereas $M_{\lambda}(f)$ does not, and, in fact, we are not aware of corresponding estimates for the Moreau envelope $M_{\lambda}(f)$.

Part (iii): This is a direct consequence of the characterisation (1.6) of the lower transform as a critical mixed Moreau envelope and the proof of Theorem 3.5.8 of Ref. 12.

Proof of Theorem 2.15. Without loss of generality we may assume that $x=0$. Since $f$ is bounded and continuous, the function $y \mapsto f(y)+\lambda|y|^{2}$ is continuous and coercive. Therefore, by Proposition 2.1,

$$
C_{\lambda}^{l}(f)(0)=\operatorname{co}\left[f+\lambda|\cdot|^{2}\right](0)=\sum_{i=1}^{n+1} \lambda_{i}\left(f\left(x_{i}\right)+\lambda\left|x_{i}\right|^{2}\right)
$$


for some $\lambda_{i} \geq 0$ and $x_{i} \in \mathbb{R}^{n}, i=1,2, \ldots, n+1$ such that $\sum_{i=1}^{n+1} \lambda_{i}=1$ and $\sum_{i=1}^{n+1} \lambda_{i} x_{i}=0$. Now, on one hand, we have the upper bound

$$
C_{\lambda}^{l}(f)(0) \leq f(0),
$$

whereas on the other hand,

(by $(2.15)$ )

$$
\begin{aligned}
C_{\lambda}^{l}(f)(0) & =\sum_{i=1}^{n+1} \lambda_{i}\left(f\left(x_{i}\right)+\lambda\left|x_{i}\right|^{2}\right) \\
& \geq f(0)-\sum_{i=1}^{n+1} \lambda_{i} \omega_{f}\left(\left|x_{i}\right|\right)+\lambda \sum_{i=1}^{n+1} \lambda_{i}\left|x_{i}\right|^{2}
\end{aligned}
$$

(by $(2.18)$ and concavity of $\omega_{f}^{c}$ )

$$
\geq f(0)-\omega_{f}^{c}\left(\sum_{i=1}^{n+1} \lambda_{i}\left|x_{i}\right|\right)+\lambda \sum_{i=1}^{n+1} \lambda_{i}\left|x_{i}\right|^{2}
$$

(by $(2.17)$ )

$$
\begin{aligned}
& \geq f(0)-a \sum_{i=1}^{n+1} \lambda_{i}\left|x_{i}\right|-b+\lambda \sum_{i=1}^{n+1} \lambda_{i}\left|x_{i}\right|^{2} \\
& =f(0)+\lambda \sum_{i=1}^{n+1} \lambda_{i}\left(\left|x_{i}\right|-\frac{a}{2 \lambda}\right)^{2}-\frac{a^{2}}{4 \lambda}-b .
\end{aligned}
$$

By comparing (7.7) and (7.8), we find

$$
\sum_{i=1}^{n+1} \lambda_{i}\left(\left|x_{i}\right|-\frac{a}{2 \lambda}\right)^{2} \leq \frac{a^{2}}{4 \lambda^{2}}+\frac{b}{\lambda} .
$$

Now using the Cauchy-Schwarz inequality, it follows that

$$
\begin{gathered}
\left(\sum_{i=1}^{n+1} \lambda_{i}\left|x_{i}\right|\right)-\frac{a}{2 \lambda}=\sum_{i=1}^{n+1} \lambda_{i}\left(\left|x_{i}\right|-\frac{a}{2 \lambda}\right) \leq \sum_{i=1}^{n+1} \lambda_{i}|| x_{i}\left|-\frac{a}{2 \lambda}\right|=\sum_{i=1}^{n+1} \lambda_{i}^{1 / 2} \lambda_{i}^{1 / 2}|| x_{i}\left|-\frac{a}{2 \lambda}\right| \\
\leq\left(\sum_{i=1}^{n+1} \lambda_{i}\right)^{1 / 2}\left(\sum_{i=1}^{n+1} \lambda_{i}\left(\left|x_{i}\right|-\frac{a}{2 \lambda}\right)^{2}\right)^{1 / 2} \leq\left(\frac{a^{2}}{4 \lambda^{2}}+\frac{b}{\lambda}\right)^{1 / 2} \leq \frac{a}{2 \lambda}+\sqrt{\frac{b}{\lambda}}
\end{gathered}
$$

so that

$$
\sum_{i=1}^{n+1} \lambda_{i}\left|x_{i}\right| \leq \frac{a}{\lambda}+\sqrt{\frac{b}{\lambda}}
$$

Since $\omega_{f}^{c}$ is non-decreasing, from (7.10) and the first part of (7.8) we have

$$
C_{\lambda}^{l}(f)(0) \geq f(0)-\omega_{f}^{c}\left(\sum_{i=1}^{n+1} \lambda_{i}\left|x_{i}\right|\right)+\lambda \sum_{i=1}^{n+1} \lambda_{i}\left|x_{i}\right|^{2} \geq f(0)-\omega_{f}^{c}\left(\frac{a}{\lambda}+\sqrt{\frac{b}{\lambda}}\right),
$$

which concludes the proof. The proof for the upper transform follows similar arguments.

Proof of Proposition 3.1. By definition of $f(x)$ as pointwise supremum of the 
lower semicontinuous functions that are lower than $f$, we have $\underline{f}(x) \leq f(x)$ for all $x \in \mathbb{R}^{n}$, hence $C_{\lambda}^{l}(f)(x) \leq C_{\lambda}^{l}(f)(x)$. Next we prove the opposite inequality. By definition, ${ }^{23,34}$

$\operatorname{co}\left[f+\lambda|\cdot|^{2}\right](x)=\sup \left\{\ell(x): \ell(y) \leq f(y)+\lambda|y|^{2} \quad\right.$ for all $\left.y \in \mathbb{R}^{n}, \quad \ell \in \operatorname{Aff}\left(\mathbb{R}^{n}\right)\right\}$

Now for every $\ell \in \operatorname{Aff}\left(\mathbb{R}^{n}\right)$ such that $\ell(y) \leq f(y)+\lambda|y|^{2}$ for all $y \in \mathbb{R}^{n}$, it is easy to see that $\ell(y) \leq \underline{f}(y)+\lambda|y|^{2}$ for all $y \in \mathbb{R}^{n}$. Hence

$$
\operatorname{co}\left[f+\lambda|\cdot|^{2}\right](x) \leq \operatorname{co}\left[\underline{f}+\lambda|\cdot|^{2}\right](x) \text { for all } x \in \mathbb{R}^{n},
$$

and therefore

$$
C_{\lambda}^{l}(f)(x) \leq C_{\lambda}^{l}(\underline{f})(x),
$$

thus, $C_{\lambda}^{l}(f)=C_{\lambda}^{l}(f)$. By passing to the limit for $\lambda \rightarrow \infty$, and recalling Theorem 2.3 of Ref. 48, given that $f$ is lower semicontinuous, we obtain that for each $x \in \mathbb{R}^{n}$

$$
\lim _{\lambda \rightarrow \infty} C_{\lambda}^{l}(\underline{f})(x)=\lim _{\lambda \rightarrow \infty} C_{\lambda}^{l}(f)(x)=\underline{f}(x),
$$

which concludes the proof for the lower transform. The proof for the upper transform follows similar arguments.

We report next the proof of Lemma 3.9, whose arguments are then used also in the proofs of Theorem 3.4 and Theorem 3.10 .

Proof of Lemma 3.9. Since $E$ is a closed set, $-\chi_{E}$ is lower semicontinuous and so is $f_{\lambda}$, hence from Proposition 2.1, there exist $\left(\tau_{i}, x_{i}\right) \in \mathbb{R} \times \mathbb{R}^{n}$ for $i=1, \ldots, n+1$, with $\sum \tau_{i} x_{i}=0, \sum \tau_{i}=1$ and $\tau_{i} \geq 0$, and a hyperplane $\ell$, say $\ell(x)=a \cdot x+b$ with some $a \in \mathbb{R}^{n}$ and $b \in \mathbb{R}$, such that

$$
\ell(x) \leq f_{\lambda}(x) \quad \text { for } x \in \mathbb{R}^{n} \quad \text { and } \quad \ell\left(x_{i}\right)=f_{\lambda}\left(x_{i}\right) \quad \text { for } \quad i=1, \ldots, n+1 .
$$

From (7.16) it follows

$$
\ell\left(x_{i}\right)=\operatorname{co}\left[f_{\lambda}\right]\left(x_{i}\right) \text { for } i=1, \ldots, n+1,
$$

and given that $\operatorname{co}\left[f_{\lambda}\right](0)=\sum_{i=1}^{n+1} \tau_{i} f_{\lambda}\left(x_{i}\right)$, then from (7.16), we have $\operatorname{co}\left[f_{\lambda}\right](0)=\ell(0)$, that is,

$$
\operatorname{co}\left[f_{\lambda}\right](0)=b .
$$

Since $0 \leq \chi_{E}(x) \leq 1$ for all $x \in \mathbb{R}^{n}$, then

$$
\lambda|x|^{2}-1 \leq \operatorname{co}\left[f_{\lambda}\right](x) \leq f_{\lambda}(x) \leq \lambda|x|^{2} \quad \text { for all } x \in \mathbb{R}^{n}
$$

where we have used the fact that co $f_{\lambda}$ is the pointwise supremum of the convex functions below $f_{\lambda}$ and $\lambda|x|^{2}-1 \leq f_{\lambda}(x)$ for all $x \in \mathbb{R}^{n}$. Hence, for $x=0$ and by accounting for (7.18) we obtain

$$
-1 \leq b \leq 0
$$


From (7.16), at the supporting points $x_{i}$ we have

$$
\lambda\left|x_{i}\right|^{2}-\chi_{E}\left(x_{i}\right)=a \cdot x_{i}+b \quad \text { for } i=1, \ldots, n+1,
$$

thus,

$$
\lambda\left|x_{i}\right|^{2} \leq|a| \cdot\left|x_{i}\right|+1 \quad \text { for } i=1, \ldots, n+1
$$

where we have used the fact that $\chi_{E}(x)+b \leq 1$ for all $x \in \mathbb{R}^{n}$ and the CauchySchwarz inequality. To obtain now an estimate on $|a|$, we note that since $\ell(x) \leq$ $f_{\lambda}(x)$ for all $x \in \mathbb{R}^{n}$, in particular, then, for $x=a /(2 \lambda)$ we deduce

$$
|a| \leq 2 \sqrt{\lambda} .
$$

By combining (7.22) and (7.23) we obtain, therefore, that there must hold

$$
\lambda\left|x_{i}\right|^{2}-2 \sqrt{\lambda}\left|x_{i}\right|-1 \leq 0 \quad \text { for } i=1, \ldots, n+1
$$

hence,

$$
\left|x_{i}\right| \leq \frac{1+\sqrt{2}}{\sqrt{\lambda}} \text { for } i=1, \ldots, n+1,
$$

which concludes the proof.

Proof of Theorem 3.2. Part (i): Since obviously $E \cap E^{c}=\varnothing$ and $E \cup E^{c}=\mathbb{R}^{n}$, we have $\chi_{E}(x)+\chi_{E^{c}}(x)=1$ for $x \in \mathbb{R}^{n}$. Thus

$$
\begin{aligned}
& C_{\lambda}^{u}\left(\chi_{E}\right)(x)=C_{\lambda}^{u}\left(1-\chi_{E^{c}}\right)(x)=\lambda|x|^{2}-\operatorname{co}\left[\lambda|\cdot|^{2}-1+\chi_{E^{c}}\right](x) \\
& =\lambda|x|^{2}+1-\operatorname{co}\left[\lambda|\cdot|^{2}+\chi_{E^{c}}\right](x)=1-C_{\lambda}^{l}\left(\chi_{E^{c}}\right)(x) .
\end{aligned}
$$

Part (ii): This is a direct consequence of Proposition 3.1 as $\bar{\chi}_{E}=\chi_{\bar{E}}$.

Part (iii): If $\overline{E^{c}}=\mathbb{R}^{n}$, then by (ii) and the same definition (1.3) of $C_{\lambda}^{u}$, we have

$$
C_{\lambda}^{u}\left(\chi_{E^{c}}\right)(x)=C_{\lambda}^{u}\left(\chi_{\overline{E^{c}}}\right)(x)=C_{\lambda}^{u}\left(\chi_{\mathbb{R}^{n}}\right)(x)=1 .
$$

By $(i)$, we have $C_{\lambda}^{l}\left(\chi_{E}\right)(x)=1-C_{\lambda}^{u}\left(\chi_{E^{c}}\right)(x)=1-1=0$, which completes the proof.

Proof of Theorem 3.4. Note first that by Theorem 3.2(ii), it suffices to prove the result when $E=\bar{E}$. We use the characterisation (1.5). If $x \in E$, then

$$
1=f(x) \leq C_{\lambda}^{u}(f)(x) \leq \lambda|y-x|^{2}+\left.1\right|_{y=x}=1,
$$

so that $C_{\lambda}^{u}(f)(x)=1$. If $x \in\left(E^{1 / \sqrt{\lambda}}\right)^{c}$, then for each $y \in E,|x-y|^{2} \geq \frac{1}{\lambda}$, so that $\lambda|x-y|^{2} \geq 1=f(y)$. Hence

$$
0 \leq C_{\lambda}^{u}(f(x)) \leq\left.\lambda|x-y|^{2}\right|_{y=x}=0
$$


and thus $C_{\lambda}^{u}(f)(x)=0$. Finally, take $x \in\left(E^{1 / \sqrt{\lambda}}\right) \backslash E$. Then there exist $\hat{x} \in E$ with $|x-\hat{x}|<\frac{1}{\sqrt{\lambda}}$ and $\hat{y} \in\left(E^{1 / \sqrt{\lambda}}\right)^{c}$ with $|x-\hat{y}|<\frac{1}{\sqrt{\lambda}}$. Since, as above, we have $\lambda|x-\hat{y}| \geq f(y)$ for all $y \in \mathbb{R}^{n}$, it follows that

$$
C_{\lambda}^{u}\left(\chi_{E}\right)(x) \leq \lambda|x-\hat{y}|^{2}<1 .
$$

On the other hand, if $C_{\lambda}^{u}\left(\chi_{E}\right)(x)=0$, then it follows from (1.5) that there exist $x_{k} \rightarrow x$ and $c_{k} \rightarrow 0$ such that $\chi_{E}(y) \leq \lambda\left|y-x_{k}\right|^{2}+c_{k}$ for all $y \in \mathbb{R}^{n}$, and hence $\chi_{E}(y) \leq \lambda|x-y|^{2}$ for all $y \in \mathbb{R}^{n}$, so that

$$
1=\chi_{E}(\hat{x}) \leq \lambda|x-\hat{x}|^{2}<1
$$

which is impossible. So $C_{\lambda}^{u}\left(\chi_{E}\right)(x)>0$.

Proof of Proposition 3.5. Part $(i)$ : For any $x \in \partial E^{1 / \sqrt{\lambda}}$, the definition of $E^{1 / \sqrt{\lambda}}$ gives that $B(x ; 1 / \sqrt{\lambda}) \cap E=\varnothing$ and thus $E \subseteq B^{c}(x ; 1 / \sqrt{\lambda})$, so that $\chi_{E}(y) \leq$ $\chi_{B^{c}(x ; 1 / \sqrt{\lambda})}(y)$ for all $y \in \mathbb{R}^{n}$. Then applying the upper transform with any $\lambda>0$, we have

$$
C_{\lambda}^{u}\left(\chi_{E}\right)(y) \leq C_{\lambda}^{u}\left(\chi_{B^{c}(x ; 1 / \sqrt{\lambda})}\right)(y) \text { for all } y \in \mathbb{R}^{n} .
$$

In particular, for $y=x \in \partial E^{1 / \sqrt{\lambda}}$, since $x \in \partial B^{c}(x ; 1 / \sqrt{\lambda})$, then applying Theorem 3.4 to $B^{c}(x ; 1 / \sqrt{\lambda})$ yields

$$
C_{\lambda}^{u}\left(\chi_{B^{c}(x ; 1 / \sqrt{\lambda})}\right)(x)=0 .
$$

Part (ii): First note that $\chi_{B^{c}(x ; 1 / \sqrt{\lambda})}(y) \leq \lambda|y-x|^{2}$ for all $y \in \mathbb{R}^{n}$. So the characterisation (1.5) yields that $C_{\lambda}^{u}\left(\chi_{B^{c}(x ; 1 / \sqrt{\lambda})}\right)(y) \leq \lambda|y-x|^{2}$ for all $y \in \mathbb{R}^{n}$, and hence

$$
0 \leq \frac{C_{\lambda}^{u}\left(\chi_{E}\right)(y)-C_{\lambda}^{u}\left(\chi_{E}\right)(y)}{|y-x|} \leq \lambda|y-x|
$$

which, for $y \rightarrow x$, shows that $C_{\lambda}^{u}\left(\chi_{E}\right)(y)$ is differentiable at $x$ and $D C_{\lambda}^{u}\left(\chi_{E}\right)(x)=0$.

Proof of Theorem 3.7. Part ( $i$ ): Without loss of generality, we can assume $x_{1}=0$ and take $E:=\bar{\Omega}$. Let us then consider the function $\lambda|y|^{2}-\chi_{E}(y)$ and define $\ell(y) \equiv 0$ as the proposed supporting affine function. First we show that

$$
f_{\lambda}(y):=\lambda|y|^{2}-\chi_{E}(y) \geq \ell(y)=0 \quad \text { for all } y \in \mathbb{R}^{n} .
$$

For this purpose, we distinguish the following two cases.

Case $(a): y \in B\left(x_{1} ; \delta\right)$. In this case $\chi_{E}(y)=0$ hence

$$
f_{\lambda}(y)=\lambda|y|^{2}-\chi_{E}(y)=\lambda|y|^{2} \geq \ell(y)=0 .
$$

Case $(b): y \notin B\left(x_{1} ; \delta\right)$. In this case $\lambda|y|^{2} \geq \lambda \delta^{2} \geq 1$, hence

$$
f_{\lambda}(y)=\lambda|y|^{2}-\chi_{E}(y) \geq \lambda \delta^{2}-1 \geq \ell(y)=0 .
$$


Next we have $f_{\lambda}(0)=0=\ell(0)$ and, since $x \in E$,

$$
f_{\lambda}(x)=\lambda|x|^{2}-1=\lambda(1 / \sqrt{\lambda})^{2}-1=0=\ell(x) .
$$

Therefore $\operatorname{co}\left[f_{\lambda}\right](y)=\ell(y)=0$ for $y \in[0, x]$, hence $C_{\lambda}^{u}\left(\chi_{E}\right)(y)=\lambda|y|^{2}$ for $y \in[0, x]$. For every point $y \in\left[x_{0}, x_{1}\right]$, we have $y \notin E^{1 \sqrt{\lambda}}$, thus $C_{\lambda}^{u}\left(\chi_{E}\right)(y)=0$.

Part (ii): By using the identity $C_{\lambda}^{u}\left(\chi_{E}\right)+C_{\lambda}^{l}\left(\chi_{E^{c}}\right) \equiv 1$, we can then apply the same arguments as in Part (i) and conclude the proof.

Proof of Theorem 3.8. We fix $x_{0} \in \mathbb{R}^{n}$ and apply Taylor's expansion to $g$ at $x_{0}$. We have

$$
g(x)=g\left(x_{0}\right)+D g\left(x_{0}\right) \cdot\left(x-x_{0}\right)+\int_{0}^{1}\left[D g\left(x_{0}+t\left(x-x_{0}\right)\right)-D g\left(x_{0}\right)\right] \cdot\left(x-x_{0}\right) d t .
$$

By our assumption on $g$, we have the following estimates:

$g\left(x_{0}\right)+D g\left(x_{0}\right) \cdot\left(x-x_{0}\right)-\frac{\lambda_{0}}{2}\left|x-x_{0}\right|^{2} \leq g(x) \leq g\left(x_{0}\right)+D g\left(x_{0}\right) \cdot\left(x-x_{0}\right)+\frac{\lambda_{0}}{2}\left|x-x_{0}\right|^{2}$.

If we let $\ell(x)=g\left(x_{0}\right)+D g\left(x_{0}\right) \cdot\left(x-x_{0}\right)$, clearly $\ell$ is affine and $\ell\left(x_{0}\right)=g\left(x_{0}\right)$, thus we obtain

$$
C_{\frac{\lambda_{0}}{2}}^{l}(g)\left(x_{0}\right)=g\left(x_{0}\right)=C_{\frac{\lambda_{0}}{2}}^{u}(g)\left(x_{0}\right) .
$$

By applying then (2.7) once to $f$ and $g$, and once to $f+g$ and to $-g$, and by accounting for (7.36), we easily conclude with (3.6).

Proof of Theorem 3.10. Part (i): We prove our result for the lower transform; the proof for the upper transform is similar. Recall from (1.6) that $C_{\lambda}^{l}(f)(x)=$ $M^{\lambda}\left(M_{\lambda}(f)\right)(x)$. If $y, z \in \mathbb{R}^{n}$ are such that $|y-z|^{2}>\frac{2 M}{\lambda}$, then

$$
f(z)+\lambda|y-z|^{2}>-M+2 M=M \geq f(y),
$$

and hence

$M_{\lambda}(f)(y)=\inf \left\{f(z)+\lambda|y-z|^{2}, z \in \mathbb{R}^{n}\right\}=\inf \left\{f(z)+\lambda|y-z|^{2}, z \in B\left(y ; \sqrt{\frac{2 M}{\lambda}}\right)\right\}$.

Moreover, since $\left|M_{\lambda}(f)(x)\right| \leq M$, it follows that for $x, y \in \mathbb{R}^{n}$ with $|x-y|^{2}>\frac{2 M}{\lambda}$, we have

$$
M_{\lambda}(f)(y)-\lambda|x-y|^{2}<M-2 M=-M \leq M_{\lambda}(f)(x)
$$

so that

$$
\begin{aligned}
& M^{\lambda}\left(M_{\lambda}(f)\right)(x)=\sup \left\{M_{\lambda}(f)(y)-\lambda|x-y|^{2}, y \in \mathbb{R}^{n}\right\} \\
& \quad=\sup \left\{M_{\lambda}(f)(y)-\lambda|x-y|^{2}, y \in B\left(x ; \sqrt{\frac{2 M}{\lambda}}\right)\right\} \\
& \quad=\sup \left\{\inf \left\{f(z)+\lambda|y-z|^{2}, z \in B\left(y ; \sqrt{\frac{2 M}{\lambda}}\right)\right\}-\lambda|x-y|^{2}, y \in B\left(x ; \sqrt{\frac{2 M}{\lambda}}\right)\right\} .
\end{aligned}
$$

Thus $C_{\lambda}^{l}(f)(x)=M^{\lambda}\left(M_{\lambda}(f)(x)\right.$ depends only on points in the ball $B\left(x ; 2 \sqrt{\frac{2 M}{\lambda}}\right)$. 
Part (ii): By Proposition 3.1, $C_{\lambda}^{l}(f)=C_{\lambda}^{l}(f)$ hence, the assumption of this part of the theorem reads also as $C_{\lambda}^{l}(\underline{f})\left(x_{0}\right)<\underline{f}\left(x_{0}\right)$ and, by accounting for $(2.8)$, this can also be expressed as

$$
C_{\lambda}^{l}(\underline{f})\left(x_{0}\right)=\operatorname{co}\left[\lambda\left|(\cdot)-x_{0}\right|^{2}+\underline{f}\right]\left(x_{0}\right)<\underline{f}\left(x_{0}\right) .
$$

Now, let $f_{\lambda}=\lambda\left|x-x_{0}\right|^{2}+\underline{f}(x)$ and observe that $f_{\lambda}$ is lower semicontinuous and coercive, hence, for the Proposition 2.1, there are $x_{i} \in \mathbb{R}^{n}$ with $x_{i} \neq x_{0}$ because of (7.37), which belong to $B\left(x_{0} ; R_{\lambda, M}\right)$ in virtue of Part $(i)$ of this theorem, and $\lambda_{i} \geq 0$ for $i=1, \ldots, n+1$ satisfying $\sum_{i=1}^{n+1} \lambda_{i}=1, \sum_{i=1}^{n+1} \lambda_{i} x_{i}=x_{0}$, and a supporting hyperplane $\ell(x)=a \cdot x+b$ such that

$$
\ell\left(x_{i}\right)=f_{\lambda}\left(x_{i}\right) \text { for } i=1, \ldots, n+1, \quad \text { and } \ell(x) \leq f_{\lambda}(x) \text { for all } x \in \mathbb{R}^{n} .
$$

Hence, we have

$$
\operatorname{co}\left[f_{\lambda}\right]\left(x_{i}\right)=\ell\left(x_{i}\right) \text { for } i=1, \ldots, n+1,
$$

that is, by accounting for (7.38),

$\operatorname{co}\left[\lambda\left|(\cdot)-x_{0}\right|^{2}+\underline{f}\right]\left(x_{i}\right)=\ell\left(x_{i}\right)=f_{\lambda}\left(x_{i}\right)=\lambda\left|x_{i}-x_{0}\right|^{2}+\underline{f}\left(x_{i}\right) \quad$ for $i=1, \ldots, n+1$.

Since the convex envelope is invariant with respect to affine functions, we observe that

$\operatorname{co}\left[\lambda\left|(\cdot)-x_{i}+x_{i}-x_{0}\right|^{2}+\underline{f}\right](x)=\operatorname{co}\left[\lambda\left|(\cdot)-x_{i}\right|^{2}+\underline{f}\right](x)+\lambda\left|x_{i}-x_{0}\right|^{2}+2 \lambda\left(x_{i}-x_{0}\right) \cdot\left(x-x_{i}\right)$,

therefore, for $x=x_{i}$, we obtain

$$
\operatorname{co}\left[\lambda\left|(\cdot)-x_{0}\right|^{2}+\underline{f}\right]\left(x_{i}\right)=\operatorname{co}\left[\lambda\left|(\cdot)-x_{i}\right|^{2}+\underline{f}\right]\left(x_{i}\right)+\lambda\left|x_{i}-x_{0}\right|^{2} .
$$

By comparing (7.39) and (7.40) we have for $i=1, \ldots, n+1$,

$\operatorname{co}\left[\lambda\left|(\cdot)-x_{0}\right|^{2}+\underline{f}\right]\left(x_{i}\right)=\operatorname{co}\left[\lambda\left|(\cdot)-x_{i}\right|^{2}+\underline{f}\right]\left(x_{i}\right)+\lambda\left|x_{i}-x_{0}\right|^{2}=f_{\lambda}\left(x_{i}\right)=\lambda\left|x_{i}-x_{0}\right|^{2}+\underline{f}\left(x_{i}\right)$

that is,

$$
C_{\lambda}^{u}(\underline{f})\left(x_{i}\right)=\operatorname{co}\left[\lambda\left|(\cdot)-x_{i}\right|^{2}+\underline{f}\right]\left(x_{i}\right)=\underline{f}\left(x_{i}\right) \quad \text { for } i=1, \ldots, n+1,
$$

which concludes the proof.

Proof of Theorem 3.13. Part (i): We prove the case for the lower transform $C_{\lambda}^{l}(f)(x)$. The proof for the upper transform is similar. Since $|f(x)| \leq M$ for $x \in \mathbb{R}^{n}$, we see that $\left|C_{\lambda}^{l}(f)(x)\right| \leq M$ for $x \in \mathbb{R}^{n}$. Fix $x_{0} \in \mathbb{R}^{n}$ and let $r>0$ to be determined, we have

$\operatorname{Lip}\left(C_{\lambda}^{l}(f), B\left(x_{0} ; r\right)\right) \leq \operatorname{Lip}\left(\operatorname{co}\left[f+\lambda\left|(\cdot)-x_{0}\right|^{2}\right], B\left(x_{0} ; r\right)\right)+\operatorname{Lip}\left(\operatorname{co}\left[\lambda\left|(\cdot)-x_{0}\right|^{2}\right], B\left(x_{0} ; r\right)\right)$.

By Propostion 2.4, we have

$\operatorname{Lip}\left(\operatorname{co}\left[f+\lambda\left|(\cdot)-x_{0}\right|^{2}\right], B\left(x_{0} ; r\right)\right) \leq \frac{\operatorname{osc}\left(\operatorname{co}\left[f+\lambda\left|(\cdot)-x_{0}\right|^{2}\right], B\left(x_{0} ; 2 r\right)\right)}{r} \leq \frac{2 M+4 \lambda r^{2}}{r}$, 
given that

$$
\max _{x \in B\left(x_{0} ; 2 r\right)}\left\{\operatorname{co}\left[f+\lambda\left|(\cdot)-x_{0}\right|^{2}\right](x)\right\} \leq M+4 \lambda r^{2}, \quad \min _{x \in B\left(x_{0} ; 2 r\right)}\left\{\operatorname{co}\left[f+\lambda\left|(\cdot)-x_{0}\right|^{2}\right](x)\right\} \geq-M .
$$

Moreover,

$$
\operatorname{Lip}\left(\operatorname{co}\left[\lambda\left|(\cdot)-x_{0}\right|^{2}\right], B\left(x_{0} ; r\right)\right) \leq 2 \lambda r
$$

and thus

$$
\operatorname{Lip}\left(C_{\lambda}^{l}(f), B\left(x_{0} ; r\right)\right) \leq \frac{2 M}{r}+\frac{6 \lambda}{r} .
$$

If $\left|x-x_{0}\right| \geq r$, as $\left|C_{\lambda}^{l}(f)(x)\right|$ is bounded above by $M$, we have

$$
\frac{\left|C_{\lambda}^{l}(f)(x)-C_{\lambda}^{l}(f)\left(x_{0}\right)\right|}{\left|x-x_{0}\right|} \leq \frac{2 M}{r} .
$$

So if we take $r=\sqrt{M /(3 \lambda)}$, we then see that for any $x \in \mathbb{R}^{n}$,

$$
\left|C_{\lambda}^{l}(f)(x)-C_{\lambda}^{l}(f)\left(x_{0}\right)\right| \leq 4 \sqrt{3 M \lambda} \leq 8 \sqrt{M \lambda} .
$$

Part (ii): Since both $C_{\lambda}^{l}(f)$ and $C_{\lambda}^{u}(f)$ are Lipschitz functions with Lipschitz constants bounded above by $8 \sqrt{M \lambda}$, by Theorem 3.12 the Lipschitz constants for $C_{\tau}^{u}\left(C_{\lambda}^{l}(f)\right)$ and $C_{\tau}^{l}\left(C_{\lambda}^{u}(f)\right)$ are then bounded by $8 \sqrt{M \lambda}$, and the inequalities in (3.11) follow from Theorem 2.12(iii).

Proof of Proposition 4.3. Part (i): We only consider $R_{\lambda}(f)$, as the other case is similar. For given $x \in \mathbb{R}^{n}$ and $\ell \in \operatorname{Aff}\left(\mathbb{R}^{n}\right)$, it follows from Proposition 2.10 that

$$
C_{\lambda}^{l}(f+\ell)(x)=C_{\lambda}^{l}(f)(x)+\ell(x)
$$

and by the Definition 4.1 of $R_{\lambda}(f)$ we obtain

$$
R_{\lambda}(f+\ell)(x)=f(x)+\ell(x)-\left(C_{\lambda}^{l}(f)(x)+\ell(x)\right)=f(x)-C_{\lambda}^{l}(f)(x)=R_{\lambda}(f)(x),
$$

which concludes the proof.

Part (ii): For given $x \in \mathbb{R}^{n}$ and $\alpha>0$

$$
\begin{aligned}
& \text { co }\left[\alpha f+\lambda|\cdot|^{2}\right](x)=\sup \left\{r(x): r(y) \leq \alpha f(y)+\lambda|y|^{2} \text { for all } y \in \mathbb{R}^{n} \text { and } r \in \operatorname{Aff}\left(\mathbb{R}^{n}\right)\right\} \\
& =\sup \left\{\alpha \ell(x): \alpha \ell(y) \leq \alpha\left(f(y)+\frac{\lambda}{\alpha}|y|^{2}\right) \text { for all } y \in \mathbb{R}^{n} \text { and } \ell \in \operatorname{Aff}\left(\mathbb{R}^{n}\right)\right\} \\
& =\alpha \sup \left\{\ell(x): \ell(y) \leq f(y)+\frac{\lambda}{\alpha}|y|^{2} \text { for all } y \in \mathbb{R}^{n} \text { and } \ell \in \operatorname{Aff}\left(\mathbb{R}^{n}\right)\right\} \\
& =\alpha \operatorname{co}\left[f+\frac{\lambda}{\alpha}|\cdot|^{2}\right](x) .
\end{aligned}
$$


Thus

$$
C_{\lambda}^{l}(\alpha f)(x)=\alpha\left(\operatorname{co}\left[f+\frac{\lambda}{\alpha}|\cdot|^{2}\right](x)-\frac{\lambda}{\alpha}|x|^{2}\right)
$$

so that

$$
R_{\lambda}(\alpha f)(x)=\alpha f(x)-\alpha\left(\operatorname{co}\left[f+\frac{\lambda}{\alpha}|\cdot|^{2}\right](x)-\frac{\lambda}{\alpha}|x|^{2}\right)=\alpha R_{\lambda / \alpha}(f)(x),
$$

which concludes the proof.

Proof of Proposition 4.4. Part (i): By definition

$$
\begin{aligned}
R_{\lambda}(f)(x) & =f(x)-C_{\lambda}^{l}(f)(x)=f(x)+\lambda|x|^{2}-\operatorname{co}\left[\lambda|\cdot|^{2}-(-f)\right](x) \\
& =-\left(-f(x)-C_{\lambda}^{u}(-f)(x)\right)=-V_{\lambda}(-f)(x) .
\end{aligned}
$$

The proof of the other equality is similar.

Part (ii): We first prove that $R_{\lambda+\tau}(f) \leq R_{\tau}\left(R_{\lambda}(f)\right)$. From (2.7), we have

$$
C_{\lambda+\tau}^{l}(f)=C_{\tau}^{l}\left(f-C_{\lambda}^{l}(f)\right)+C_{\lambda}^{l}\left(C_{\lambda}^{l}(f)\right)
$$

and since $C_{\lambda}^{l}\left(C_{\lambda}^{l}(f)\right)=C_{\lambda}^{l}(f)$, we conclude that

$$
\left.R_{\tau}\left(R_{\lambda}(f)\right)=\left(f-C_{\tau}^{l}(f)\right)-C_{\lambda}^{l}(f)\right)+C_{\tau}^{l}\left(f-C_{\lambda}^{l}(f)\right) \geq f-C_{\lambda+\tau}^{l}(f)=R_{\lambda+\tau}(f) .
$$

To show that $R_{\tau}\left(R_{\lambda}(f)\right)(x) \leq R_{\lambda}(f)(x)$, just observe that for any $\lambda>0$, since $R_{\lambda}(f)(x) \geq 0$, then from $(2.6)$, we have for any $\tau>0$ that $C_{\tau}^{l}\left(R_{\lambda}(f)\right)(x) \geq 0$, hence

$$
R_{\lambda}(f)(x)-C_{\tau}^{l}\left(R_{\lambda}(f)\right)(x) \leq R_{\lambda}(f)(x)
$$

which concludes the proof.

Proof of Theorem 4.6. The statements are direct consequences of Theorem 3.8.

Proof of Theorem 4.8. Part (i): Observe first that given a regular open set $\Omega$ of $\mathbb{R}^{n}$ and $\Gamma \subseteq \partial \Omega$, then it is $\underline{\chi}_{\Omega \cup \Gamma}=\chi_{\Omega}$, where we recall that $\underline{\chi}_{\Omega \cup \Gamma}$ is the lower semicontinuous envelope of $\chi_{\Omega \cup \Gamma}$. By Proposition 3.1 it thus follows that

$$
C_{\lambda}^{l}\left(\chi_{\Omega \cup \Gamma}\right)=C_{\lambda}^{l}\left(\underline{\chi}_{\Omega \cup \Gamma}\right) .
$$

By recalling the above, we have that $\underline{\chi}_{\Omega \cup \Gamma}=\chi_{\Omega}$, and hence by Theorem 3.2 we can conclude that

$$
C_{\lambda}^{l}\left(\underline{\chi}_{\Omega \cup \Gamma}\right)=1-C_{\lambda}^{u}\left(\chi_{\Omega^{c}}\right) .
$$

Now, by Theorem 3.4 we have the following characterization for $C_{\lambda}^{u}\left(\chi_{\Omega^{c}}\right)$

$$
C_{\lambda}^{u}\left(\chi_{\Omega^{c}}\right)(x) \begin{cases}=1 & \text { if } x \in \Omega^{c} \\ =0 & \text { if } x \in \Omega \backslash\left(\Omega^{c}\right)^{1 \sqrt{\lambda}} \\ \in(0,1) & \text { if } x \in\left(\Omega^{c}\right)^{1 \sqrt{\lambda}} \backslash \Omega^{c}\end{cases}
$$


and given that

$$
R_{\lambda}\left(\chi_{\Omega \cup \Gamma}\right)=\chi_{\Omega \cup \Gamma}-C_{\lambda}^{l}\left(\chi_{\Omega \cup \Gamma}\right),
$$

taking into account (7.50), (7.51), and (7.53) we can conclude that (4.4) holds. The continuity claim in $\mathbb{R}^{n} \backslash \partial \Omega$ also follows as $\chi_{\Omega}(x)$ is continuous at any $x \in \mathbb{R}^{n} \backslash \partial \Omega$. The claims for $V_{\lambda}\left(\chi_{\Omega \cup \Gamma}\right)$ are proved with similar arguments.

Part (ii): This also follows from Theorem 3.2 and Theorem 3.4. The continuity claim is a consequence of the fact that both the upper and the lower transforms are continuous in $\mathbb{R}^{n}$. Finally, we establish the limit property. We have, on $\partial \Omega$, $E_{\lambda}\left(\chi_{\Omega}\right)(x)=1$ for all $\lambda>0$ and on $\left[(\partial \Omega)^{1 / \sqrt{\lambda}}\right]^{c}, E_{\lambda}\left(\chi_{\Omega}\right)(x)=0$. Therefore for any given $x \notin \partial \Omega$, we have $x \notin\left[(\partial \Omega)^{1 / \sqrt{\lambda}}\right]^{c}$ when $\lambda>0$ is large enough. Therefore $\lim _{\lambda \rightarrow+\infty} E_{\lambda}\left(\chi_{\Omega}\right)(x)=\chi_{\partial \Omega}(x)$ for each fixed $x \in \mathbb{R}^{n}$.

Proof of Proposition 4.9. The bound (4.8) is a direct consequence of Theorem 2.12. From (4.8) and the observation that for $\lambda>1$, and $0 \leq \beta<\alpha<2$,

$$
0 \leq \liminf _{\lambda \rightarrow+\infty} \lambda^{\beta /(2-\beta)} R_{\lambda} f\left(x_{0}\right) \leq \limsup _{\lambda \rightarrow+\infty} \lambda^{\alpha /(2-\alpha)} R_{\lambda} f\left(x_{0}\right) \lim _{\lambda \rightarrow+\infty}(1 / \lambda)^{\frac{\alpha-\beta}{(2-\alpha)(2-\beta)}},
$$

it follows that $\liminf _{\lambda \rightarrow+\infty} \lambda^{\beta /(2-\beta)} R_{\lambda} f\left(x_{0}\right)=0$.

Proof of Proposition 4.10. The proof follows the arguments used to prove Proposition 4.9.

Proof of Proposition 5.2: The proof follows from the definition (5.2) of $D_{\lambda}^{2}(x, E)$.

Proof of Proposition 5.3: Since by definition (5.2), $\chi_{E}(x) \leq D_{\lambda}^{2}(x ; E)$ for $x \in \mathbb{R}^{n}$, we have, by (2.4), that

$$
0 \leq C_{\lambda}^{u}\left(\chi_{E}\right)(x) \leq C_{\lambda}^{u}\left(D_{\lambda}^{2}(\cdot ; E)\right)(x), \quad x \in \mathbb{R}^{n} .
$$

To establish the opposite inequality, let $p(x):=\lambda\left|x-x_{0}\right|^{2}+c$ be such that $p(x) \geq$ $\chi_{E}(x)$ for all $x \in \mathbb{R}^{n}$ and $p(\hat{x})=1=\chi_{E}(\hat{x})$ for some $\hat{x} \in E$. It will suffice to show that

$$
D_{\lambda}^{2}(x ; E) \leq p(x) \text { for all } x \in \mathbb{R}^{n},
$$

since the characterisation (1.5) will then yield that $C_{\lambda}^{u}\left(D_{\lambda}^{2}(\cdot ; E)\right)(x) \leq C_{\lambda}^{u}\left(\chi_{E}\right)(x)$, $x \in \mathbb{R}^{n}$. Define $R:=\sqrt{\frac{1-c}{\lambda}}$ and note first that $E \subset B\left(x_{0} ; R\right)^{c}$, since if $x \in E$, then $1 \leq \lambda\left|x-x_{0}\right|^{2}+c$, and hence $x \in B\left(x_{0} ; R\right)^{c}$. Hence, by Proposition 5.2(i), we have

$$
D^{2}(x ; E) \leq D_{\lambda}^{2}\left(x ; B\left(x_{0} ; R\right)^{c}\right) \text { for all } x \in \mathbb{R}^{n} .
$$

Now if $x \in B\left(x_{0} ; R\right)^{c}$, then $p(x) \geq 1 \geq D_{\lambda}^{2}(x ; E)$, so (7.56) clearly holds. On the other hand, if $x \in B\left(x_{0} ; R\right)$, then $D_{\lambda}\left(x ; B\left(x_{0} ; R\right)^{c}\right)=\max \{0,1-$ 
$\left.\sqrt{\lambda} \operatorname{dist}\left(x, B\left(x_{0} ; R\right)^{c}\right)\right\}=1-\sqrt{\lambda}\left(R-\left|x-x_{0}\right|\right)$, and hence

$$
\begin{aligned}
D_{\lambda}^{2}\left(x ; B\left(x_{0} ; R\right)^{c}\right) & =\left(1-\sqrt{\lambda}\left(R-\left|x-x_{0}\right|\right)\right)^{2} \\
& =\lambda\left|x-x_{0}\right|^{2}+2 \sqrt{\lambda}\left|x-x_{0}\right|(1-\sqrt{1-c})+(1-\sqrt{1-c})^{2} \\
& <\lambda\left|x-x_{0}\right|^{2}+2 \sqrt{1-c}(1-\sqrt{1-c})+(1-\sqrt{1-c})^{2} \\
& =\lambda\left|x-x_{0}\right|^{2}+c=p(x),
\end{aligned}
$$

which, together with (7.57), yields that (7.56) holds in this case also.

Proof of Lemma 5.4: We have

$$
\begin{aligned}
& \left|D_{\lambda}^{2}(x ; E)-D_{\lambda}^{2}(x ; F)\right|=\left|D_{\lambda}(x ; E)+D_{\lambda}(x ; F)\right|\left|D_{\lambda}(x ; E)-D_{\lambda}(x ; F)\right| \\
& \quad \leq 2\left|D_{\lambda}(x ; E)-D_{\lambda}(x ; F)\right| \\
& \quad=2\left|\frac{1}{2}(1-\sqrt{\lambda} \operatorname{dist}(x ; E)+|1-\sqrt{\lambda} \operatorname{dist}(x ; E)|)-\frac{1}{2}(1-\sqrt{\lambda} \operatorname{dist}(x ; F)+\mid 1-\sqrt{\lambda} \operatorname{dist}(x ; F))\right| \\
& \quad \leq 2 \sqrt{\lambda}|\operatorname{dist}(x ; E)-\operatorname{dist}(x ; F)| \leq 2 \sqrt{\lambda} \operatorname{dist}_{\mathcal{H}}(E, F),
\end{aligned}
$$

where we used $\left.\left.0 \leq D_{\lambda}(x ; E)\right) \leq 1,0 \leq D_{\lambda}(x ; F)\right) \leq 1$ and $\max \{0, a\}=(a+|a|) / 2$ for $a \in \mathbb{R}$.

Proof of Theorem 5.5: By Proposition 5.3, we only need to show that $C_{\lambda}^{u}\left(D_{\lambda}^{2}(\cdot ; E)\right)(x)$ is Hausdorff continuous. By Lemma 5.4 we have

$$
D_{\lambda}^{2}(x ; F)-2 \sqrt{\lambda} \operatorname{dist}_{\mathcal{H}}(E, F) \leq D_{\lambda}^{2}(x ; E) \leq D_{\lambda}^{2}(x ; F)+2 \sqrt{\lambda} \operatorname{dist}_{\mathcal{H}}(E, F) .
$$

Taking the upper transform in the above inequalities and using the ordered property for the upper transforms, we obtain

$C_{\lambda}^{u}\left(D_{\lambda}^{2}(\cdot ; F)-2 \sqrt{\lambda} \operatorname{dist}_{\mathcal{H}}(E, F)\right) \leq C_{\lambda}^{u}\left(D_{\lambda}^{2}(\cdot ; E)\right) \leq C_{\lambda}^{u}\left(D_{\lambda}^{2}(\cdot ; F)+2 \sqrt{\lambda} \operatorname{dist}_{\mathcal{H}}(E, F)\right)$.

By the property $\operatorname{co}[f+\ell]=\operatorname{co}[f]+\ell$ for the convex envelope when $\ell$ is an affine function, we have

$C_{\lambda}^{u}\left(D_{\lambda}^{2}(\cdot ; F)\right)-2 \sqrt{\lambda} \operatorname{dist}_{\mathcal{H}}(E, F) \leq C_{\lambda}^{u}\left(D_{\lambda}^{2}(\cdot ; E)\right) \leq C_{\lambda}^{u}\left(D_{\lambda}^{2}(\cdot ; F)\right)+2 \sqrt{\lambda} \operatorname{dist}_{\mathcal{H}}(E, F)$.

The result follows.

Proof of Corollary 5.7: Since $C_{\lambda}^{u}\left(\chi_{E}\right)$ and $C_{\lambda}^{u}\left(\chi_{F}\right)$ are bounded functions, it follows immediately from Theorem 5.5 and the fact that

$$
\left|C_{\lambda}^{l}\left(\chi_{E}\right)(x)-C_{\lambda}^{l}\left(\chi_{F}\right)(x)\right| \leq \sup _{y \in \mathbb{R}^{n}}\left|\chi_{E}(y)-\chi_{F}(y)\right| \quad\left(\text { for all } x \in \mathbb{R}^{n}\right),
$$

that for all $x \in \mathbb{R}^{n}$,

$$
\left|C_{\tau}^{l}\left(C_{\lambda}^{u}\left(\chi_{E}\right)\right)(x)-C_{\tau}^{l}\left(C_{\lambda}^{u}\left(\chi_{F}\right)\right)(x)\right| \leq \sup _{y \in \mathbb{R}^{n}}\left|C_{\lambda}^{u}\left(\chi_{E}\right)(y)-C_{\lambda}^{u}\left(\chi_{E}\right)(y)\right| \leq 2 \sqrt{\lambda} \operatorname{dist}_{\mathcal{H}}(E, F),
$$

as required. 
Proof of Theorem 5.9: The proof follows from the definition (4.1) of ridge transform, the application of the triangle inequality, of the Theorem 5.5 and of the Corollary 5.7.

Proof of Lemma 6.2: The lemma can be proved by direct calculation using Proposition 2.3.

Proof of Theorem 6.4: By comparing the expressions of the transforms for $\left.\chi_{\bar{B}(0 ; r)}\right)$ in Lemma 6.2 with those for $\chi_{K_{-}}$in Example 6.1, it is easy to see that

$$
\begin{gathered}
C_{\lambda}^{u}\left(\chi_{\bar{B}(0 ; r)}\right)(x)=C_{\lambda}^{u}\left(\chi_{K_{-}}\right)(|x|-r) ; \quad C_{\tau}^{l}\left(C_{\lambda}^{u}\left(\chi_{\bar{B}(0 ; r)}\right)\right)(x)=C_{\tau}^{l}\left(C_{\lambda}^{u}\left(\chi_{K_{-}}\right)\right)(|x|-r) ; \\
S R_{\lambda, \tau}\left(\chi_{\bar{B}(0 ; r)}\right)(x)=S R_{\lambda, \tau}\left(\chi_{K_{-}}\right)(|x|-r) .
\end{gathered}
$$

Now let $(x, y) \in \mathbb{R}^{n}$ with $x \in \mathbb{R}$ and $y \in \mathbb{R}^{n-1}$, and $\bar{B}((-r, 0) ; r)$ be the closed ball centred at $(-r, 0) \in \mathbb{R} \times \mathbb{R}^{n-1}$. If $r \geq \max \{1 / \sqrt{\lambda}, \beta\}$, then it follows from (7.59) that along the line segment $[-r, r] \times\{0\} \subset \mathbb{R}^{n}$, we have

$$
\begin{gathered}
C_{\lambda}^{u}\left(\chi_{\bar{B}((-r, 0) ; r)}\right)(t, 0)=C_{\lambda}^{u}\left(\chi_{K_{-}}\right)(t) ; \quad C_{\tau}^{l}\left(C_{\lambda}^{u}\left(\chi_{\bar{B}((-r, 0) ; r)}\right)\right)(t, 0)=C_{\tau}^{l}\left(C_{\lambda}^{u}\left(\chi_{K_{-}}\right)\right)(t) ; \\
S R_{\lambda, \tau}\left(\chi_{B((-r, 0) ; r)}\right)(t, 0)=S R_{\lambda, \tau}\left(\chi_{K_{-}}\right)(t)
\end{gathered}
$$

for $t \in[-r, r]$. The proof then follows from (7.60), a direct application of (2.5) and Lemma 6.2. We leave the details to interested readers.

Proof of Proposition 6.5: It is well-known (see, for example, Lemma 2.2 of Ref. 1) that a $C^{1,1}$ point $x_{0} \in \partial \Omega$ is an $r$-regular point for $r$ sufficiently small. Let $x^{\prime} \in \Omega$ and $r>0$ be such that $B\left(x^{\prime} ; r\right) \subset \Omega$ and $x_{0} \in \partial \Omega$. Let $x^{\prime \prime}=x_{0}-\left(x^{\prime}-x_{0}\right)$ be the opposite point of $x^{\prime}$ with respect to $x_{0}$ and consider the open ball $B\left(x^{\prime \prime} ; r\right)$. Now consider the closed set $K:=\bar{\Omega} \backslash B\left(x^{\prime \prime} ; r\right) \subset \bar{\Omega}$. Clearly $x_{0}$ is now both an interior and exterior regular point of $K$ and $x_{0} \in K$. Furthermore, $\chi_{K} \leq \chi_{\bar{\Omega}}$. By Theorem 3.4 $(i)$, we have $C_{\lambda}^{u}\left(\chi_{\Omega}\right)=C_{\lambda}^{u}\left(\chi_{\bar{\Omega}}\right)$, whereas from (2.5) we have $C_{\lambda}^{u}\left(\chi_{K}\right) \leq C_{\lambda}^{u}\left(\chi_{\bar{\Omega}}\right)$ and $C_{\tau}^{l}\left(C_{\lambda}^{u}\left(\chi_{K}\right)\right) \leq C_{\tau}^{l}\left(C_{\lambda}^{u}\left(\chi_{\bar{\Omega}}\right)\right)$. Note that since $x_{0} \in K$ and $x_{0} \in \bar{\Omega}$, we have $C_{\lambda}^{u}\left(\chi_{K}\right)\left(x_{0}\right)=C_{\lambda}^{u}\left(\chi_{\bar{\Omega}}\right)\left(x_{0}\right)=1$, hence

$$
S R_{\lambda, \tau}\left(\chi_{\Omega}\right)\left(x_{0}\right)=S R_{\lambda, \tau}\left(\chi_{\bar{\Omega}}\right)\left(x_{0}\right) \leq S R_{\lambda, \tau}\left(\chi_{K}\right)\left(x_{0}\right)=\frac{(\sqrt{\lambda+\tau}-\sqrt{\tau})^{2}}{\lambda},
$$

due to Theorem 6.4. This completes the proof.

Proof of Proposition 6.6: By Theorem 3.4(ii), $C_{\lambda}^{u}\left(\chi_{\Omega}\right)(x)=0$ if $\operatorname{dist}(x ; \bar{\Omega}) \geq$ $1 / \sqrt{\lambda}$ and $C_{\lambda}^{u}\left(\chi_{\Omega}\right)(x)=1$ if $x \in \bar{\Omega}$. To calculate $C_{\tau}^{l}\left(C_{\lambda}^{u}\left(\chi_{\Omega}\right)\right)\left(x_{0}\right)$ for $x_{0}$ satisfying $\operatorname{dist}\left(x_{0} ; \partial \Omega\right) \geq 1 / \sqrt{\lambda}$, we first observe that 0 is the minimum value of $C_{\lambda}^{u}\left(\chi_{\Omega}\right)(x)$ so that $C_{\tau}^{l}\left(C_{\lambda}^{u}\left(\chi_{\Omega}\right)\right)\left(x_{0}\right)=0$ if $\operatorname{dist}\left(x_{0} ; \bar{\Omega}\right) \geq 1 / \sqrt{\lambda}$. If $x_{0} \in \Omega$ and $\operatorname{dist}\left(x_{0} ; \partial \Omega\right) \geq$ $1 / \sqrt{\lambda}$, we consider the following function: $x \rightarrow \lambda\left|x-x_{0}\right|^{2}+C_{\lambda}^{u}\left(\chi_{\Omega}\right)(x)$, and its convex envelope at $x_{0}$. We take then the affine function $\ell(x) \equiv 1$ and observe that 
for $\left|x-x_{0}\right| \geq 1 / \sqrt{\lambda}$, we have

$$
1 \equiv \ell(x) \leq \lambda\left|x-x_{0}\right|^{2} \leq \lambda\left|x-x_{0}\right|^{2}+C_{\lambda}^{u}\left(\chi_{\Omega}\right)(x),
$$

whereas for $\left|x-x_{0}\right| \leq 1 / \sqrt{\lambda}$, we have $C_{\lambda}^{u}\left(\chi_{\Omega}\right)(x)=1$ so that

$$
1 \equiv \ell(x) \leq \lambda\left|x-x_{0}\right|^{2}+1=\lambda\left|x-x_{0}\right|^{2}+C_{\lambda}^{u}\left(\chi_{\Omega}\right)(x) .
$$

Thus $\ell(x) \equiv 1$ is a supporting affine function for $x \rightarrow \lambda\left|x-x_{0}\right|^{2}+C_{\lambda}^{u}\left(\chi_{\Omega}\right)(x)$ and this implies that $C_{\tau}^{l}\left(C_{\lambda}^{u}\left(\chi_{\Omega}\right)\right)\left(x_{0}\right)=1$, which concludes the proof.

Proof of Proposition 6.10: By a simple translation we may assume the ball is centred at 0 , so that $B(0 ; r) \subset \Omega$ and $x_{0} \in \partial B(0 ; r)$. By (6.11) in Lemma 6.2 and (2.5), we have

$C_{\lambda}^{u}\left(\chi_{\bar{B}(0 ; r)}\right)(x) \leq C_{\lambda}^{u}\left(\chi_{\bar{\Omega}}\right)(x), \quad x \in \mathbb{R}^{n}, \quad$ and $\quad C_{\lambda}^{u}\left(\chi_{\bar{B}(0 ; r)}\right)\left(x_{0}\right)=C_{\lambda}^{u}\left(\chi_{\bar{\Omega}}\right)\left(x_{0}\right)=1$,

so that

$$
C_{\tau}^{l}\left(C_{\lambda}^{u}\left(\chi_{\bar{B}_{r}(0)}\right)\left(x_{0}\right) \leq C_{\tau}^{l}\left(C_{\lambda}^{u}\left(\chi_{\bar{\Omega}}\right)\left(x_{0}\right)\right.\right.
$$

hence

$$
S R_{\lambda, \tau}\left(\chi_{\bar{\Omega}}\right)\left(x_{0}\right) \leq S R_{\lambda, \tau}\left(\chi_{\bar{B}_{r}(0)}\right)\left(x_{0}\right)=\mu_{1}(\lambda, \tau) .
$$

The last equality follows from (6.11) as $\left|x_{0}\right|=r$, and this completes the proof.

Proof of Lemma 6.13: The value of the limit (6.26) can be evaluated as a direct consequence of Example 6.11.

\section{Acknowledgment}

The authors are extremely grateful to an anonymous referee, whose constructive comments on earlier versions of the manuscript have contributed to produce a better version of the paper. Some parts of the present work were used in the UK patent application GB 0921863.7 in 2009 and the Patent Corporation Treaty (PCT) application in 2010 (WO55010). The authors wish to thank Swansea University, where this work was initiated, for its support. KZ also wishes to thank China NSF for its partial support through grant 40771094, and AO acknowledges the Argentinean Agency through the Project Prestamo BID PICT PRH 30 No 94 and the National University of Tucumán through the project PIUNT E527.

\section{References}

1. H. Aikawa, T. Kilpeläinen, N. Shanmugalingam, X. Zhong, Boundary Harnack principle for $p$-harmonic functions in smooth Euclidean domains, Potential Anal. 26 (2007) 281-301.

2. G. Alberti, On the structure of singular sets of convex functions, Calculus of Variations and Partial Differential Equations 2 (1994) 17-27. 
3. G. Alberti, L. Ambrosio, P. Cannarsa, On the singularities of convex functions, Manuscripta Math. 76 (1992) 421-435.

4. L. Ambrosio, P. Tilli, Topics on Analysis in Metric Spaces, (Oxford Univ. Press, London, 2004).

5. H. Attouch, D. Aze, Approximations and regularizations of arbitrary functions in Hilbert spaces by the Lasry-Lions methods, Anal. Non-Lin. H. Poincaré Inst. 10 (1993) 289-312.

6. D. Aussel, A. Daniilidis, L. Thibault, Subsmooth sets: functional characterizations and related concepts, Trans. Am. Math. Soc. 357 (2005) 1275-1301.

7. J. M. Ball, R. D. James, Fine phase mixtures as minimizers of energy, Arch. Rational Mech. Anal. 100 (1987) 13-52.

8. J. M. Ball, R. D. James, Proposed experimental tests of a theory of fine microstructures and the two-well problem, Phil. Royal Soc. Lond. A 338 (1992) 389-450.

9. J. M. Ball, B. Kirchheim, J. Kristensen, Regularity of quasi-convex envelopes, Calculus of Variations and Partial Differential Equations 11 (2000) 333-359.

10. R. Van Den Boomgaard, Mathematical Morphology: Extensions towards Computer Vision, (PhD Thesis, University of Amsterdam, 1992)

11. R. Van den Boomgaard, A. W. M. Smelders, The morphological structure of images, IAPR International Conference on Pattern Recognition, IEEE Computer Society Press, Los Alamitos, CA (1992) 268-271.

12. P. Cannarsa, C. Sinestrari, Semiconcave Functions, Hamilton-Jacobi Equations and Optimal Control, (Birkhäuser, Boston, 2004).

13. J. Canny, A computational approach to edge detection, IEEE Trans. Pattern Analysis and Machine Intelligence 8 (1986) 679-714.

14. M. G. Crandall, H. Ishii, P.-L. Lions, User's guide to visosity solutions of second order partial differential equations, Bull. Am. Math. Soc. 27 (1992) 1-67.

15. B. Dacorogna, Direct Methods in the Calculus of Variations, (Springer, 2nd Ed, Berlin, 2007).

16. R. A. DeVore, G. G. Lorentz, Constructive Approximation, (Springer, Berlin, 1993).

17. D. Eberly, Ridges in Image and Data Analysis, (Kluwer, Dordrecht, 1996).

18. H. Edelsbrunner, Algorithms in Combinatorial Geometry, (Springer-Verlag, Berlin, 1987).

19. D. Gilbarg, N. S. Trudinger, Elliptic Partial Differential Equations of Second Order, (Springer-Verlag, Berlin, 1998).

20. R. C. Gonzales, R. E. Woods, Digital Image Processing, (3rd Ed., Pearson Prentice Hall, New Jersey, 2008).

21. V. V. Gorokhovik, I. Zoŕoko, Piecewise affine functions and polyhedral sets, Optimizations 31 (1994) 209-221.

22. R. Haralick, Ridges and valleys on digital images, Computer Vision, Graphics, and Image Processing 22 (1983) 28-38.

23. J.-B. Hiriart-Urruty, C. Lemaréchal, Fundamentals of Convex Analysis, (Springer, Berlin, 2001).

24. C. Harris, M. Stephens, A combined corner and edge detector, Fourth Alvey Vision Conference (1988) 147-151.

25. P. T. Jackway, Morphological scale-space, IAPR International Conference on Pattern Recognition, IEEE Computer Society Press, Los Alamitos, CA (1992) 252-255.

26. J. Jost, X. Li-Jost, Calculus of Variations, (Cambridge University Press, New York, 1998).

27. B. Kirchheim, J. Kristensen, Differentiability of convex envelopes, C. R. Acad. Sci., Paris, Sér. I, Math. 333 (2001) 725-728. 
28. R. Laganière, A morphological operator for corner detection, Pattern Recognition 31 (1998) 1643-1652.

29. J. M. Lasry, P. L. Lions, A remark on regularization in Hilbert Spaces, Israel Math. J. 55 (1986) 257-266.

30. T. Lindeberg, Edge detection and ridge detection with automatic scale selection, International Journal of Computer Vision 30 (1998) 117-154.

31. J.-J. Moreau, Proximaté dualité dans un espace Hilbertien, Bull. Soc. Math. Fr. 93 (1965) 273-299.

32. J.-J. Moreau, Fonctionnelles Convexes, (Lecture Notes, Collége de France, 1966).

33. Y. G. Reshetnyak, On a generalization of convex surfaces, Mat. Sbornik 40 (1956) 381-398.

34. R. T. Rockafellar, Convex Analysis, (Princeton Univ. Press, New Jersey, 1970).

35. R. T. Rockafellar, Clarke's tangent cones and boundaries of closed sets in $\mathbb{R}^{n}$, Nonl. Anal. 3 (1979) 145-154.

36. R. T. Rockafellar, R. Wets, Variational Analysis, (Springer Verlag, Berlin, 2009).

37. S. Rolewicz, On $\gamma$-paraconvex multifunctions, Math. Jpn. 24 (1979) 293-300.

38. S. Rolewicz, On $\alpha(\cdot)$-paraconvex and strongly $\alpha$-paraconvex functions, Control Cyber. 29 (2000) 367-377.

39. J. C. Russ, The Image Processing Handbook, (5th edition, CRC Press, Boca Raton, 2007).

40. J. Serra, Image Analysis and Mathematical Morphology, (Academic Press, London, 1982).

41. K. Siddiqi, S. M. Pizer (Editors), Medial Representations, (Springer, Berlin, 2008).

42. S. Smith and J. Brady, SUSAN-a new approach to low level image processing, International Journal of Computer Vision 23 (1997) 45-78.

43. I. Sobel, An isotropic image gradient operator, In: H. Freeman (Editor), Machine Vision for Three-Dimensional Scenes, 376-379, Academic Press, 1990.

44. P. Soille, Morphological Image Analysis, (Springer, Berlin, 2nd Ed, 2004).

45. C. Tomasi, R. Manduchi, Bilateral filtering for gray and color images, In: Sixth International Conference on Computer Vision (1998) 839-846.

46. J.-P. Vial, Strong and weak convexity of sets and functions, Math. Oper. Research. 8 (1983) 231-259.

47. L. P. Yaroslavsky, Digital Picture Processing - An Introduction, (Springer, Berlin, 1985).

48. K. Zhang, Compensated convexity and its applications, Anal. Non-Lin. H. Poincaré Inst. 25 (2008) 743- 771.

49. K. Zhang, Convex analysis based smooth approximations of maximum functions and squared-distance functions, J. Nonlinear Convex Anal. 9 (2008) 379-406.

50. K. Zhang, E.C.M. Crooks, A. Orlando, Compensated convexity transforms and numerical algorithms, In preparation. 